\title{
Novel production system for influenza $A$ virus-derived defective interfering particles and analysis of antiviral activity
}

\author{
Dissertation \\ for the award of the degree \\ "Doctor rerum naturalium" \\ of the Georg-August-Universität Göttingen
}

within the doctoral program Emerging Infectious Diseases (EIDIS)

of the Georg-August University School of Science (GAUSS)

submitted by

Prerna Arora

from Sunabeda, Orissa, India

Göttingen 2020 


\section{Thesis Committee}

Prof. Dr. Stefan Pöhlmann,

Infection Biology Unit, German Primate Center

Prof. Dr. Lutz Walter,

Primate Genetics, German Primate Center

Prof. Dr. Friedemann Weber,

Institute of Virology, Veterinary Medicine of Justus Liebig University Giessen, Biomedical Research Center Seltersberg (BFS)

\section{Members of the Examination Board}

Referee: Prof. Dr. Stefan Pöhlmann,

Infection Biology Unit, German Primate Center

$2^{\text {nd }}$ Referee: Prof. Dr. Lutz Walter,

Primate Genetics, German Primate Center

\section{Further members of the Examination Board}

Prof. Dr. Friedemann Weber,

Institute of Virology, Veterinary Medicine of Justus Liebig University Giessen, Biomedical Research Center Seltersberg (BFS)

PD Dr. Christian Roos,

Primate Genetics Laboratory, German Primate Center

Dr. Alexander Hahn, Junior Research Group Herpesvirus, German Primate Center

Prof. Dr. Jörg Stülke

Institute for Microbiology and Genetics, University of Göttingen

Prof. Dr. Matthias Dobbelstein

Institute of Molecular Oncology, University Medical Center Göttingen

Date of oral examination: 27.08.2020 


\section{Affidavit}

I hereby declare that my doctoral thesis entitled "Novel production system for influenza A virus-derived defective interfering particles and analysis of antiviral activity" has been written independently with no other sources and aids than quoted.

Göttingen, July $15^{\text {th }}, 2020$

Prerna Arora 
"The single biggest threat to man's continued dominance on this planet is the virus"

Joshua Lederberg 


\section{Table of Contents}

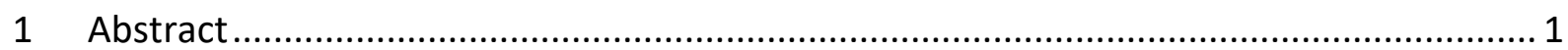

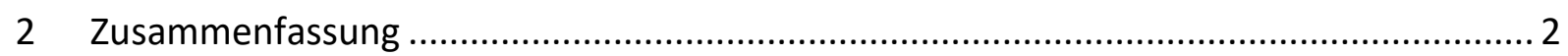

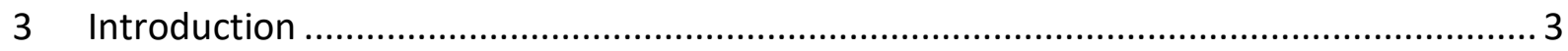

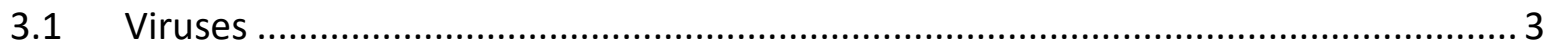

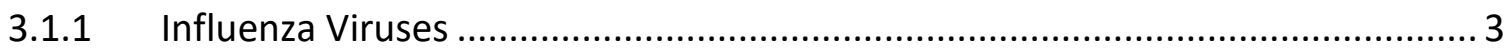

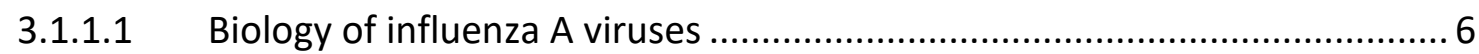

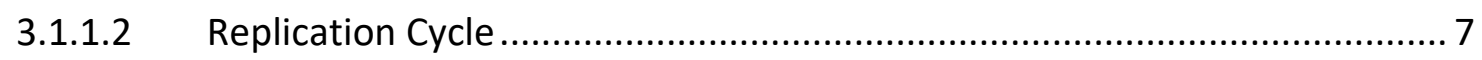

3.1.1.3 Reverse genetics system of influenza A virus .......................................... 8

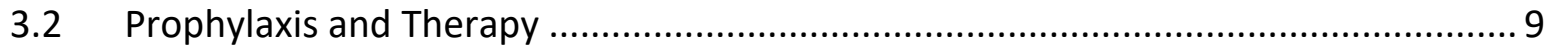

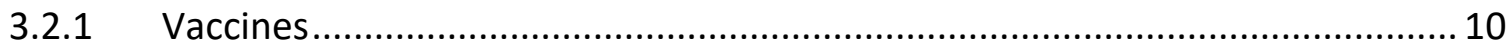

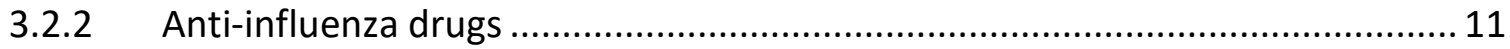

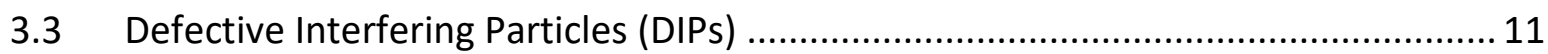

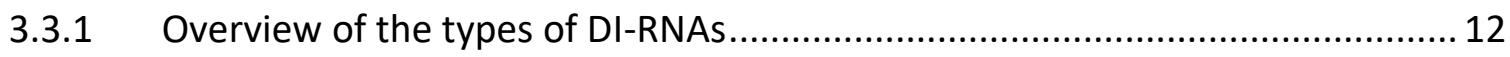

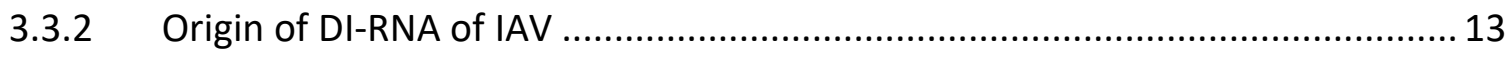

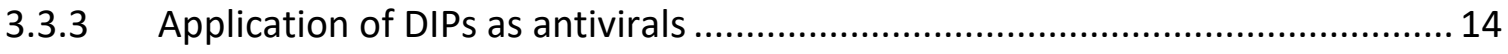

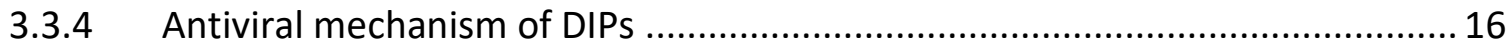

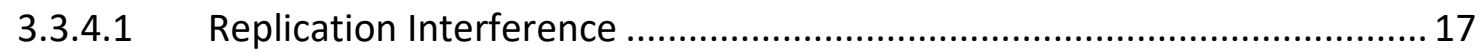

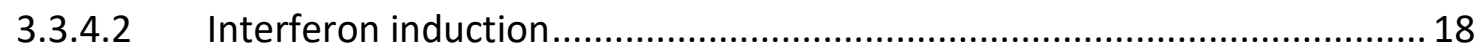

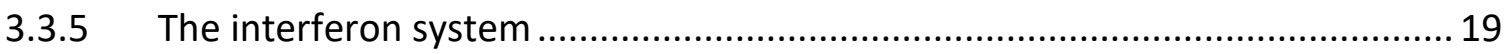

3.3.6 Sensors and signal transducers of the interferon system............................... 19

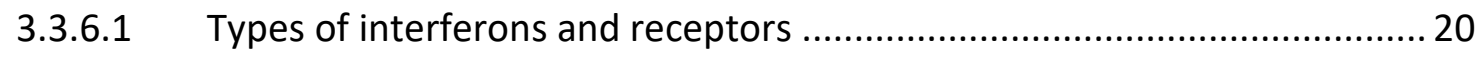

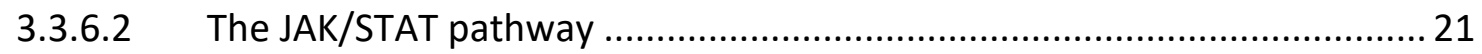

3.3.6.3 Signal Transducer and Activator of Transcription (STAT) .......................... 22

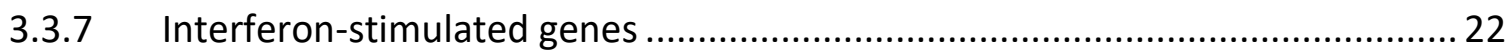

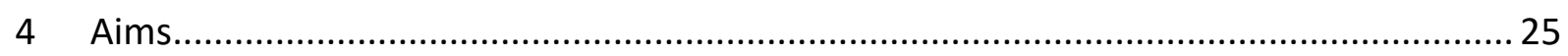

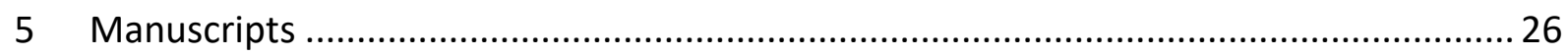

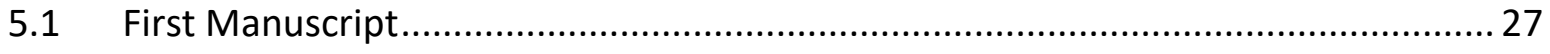

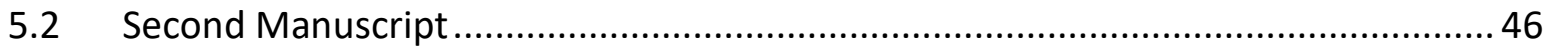

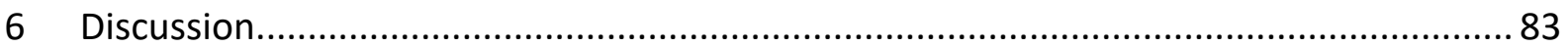

6.1 First Manuscript - A system for production of defective interfering particles in the absence of infectious influenza $A$ virus

6.2 Second Manuscript - Interferon induction and not replication interference is the major determinant of anti-influenza virus activity of defective interfering particles 


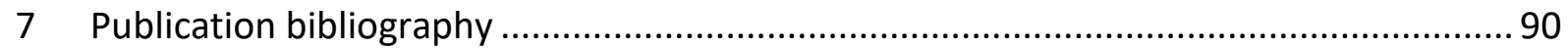

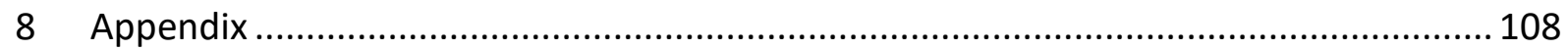

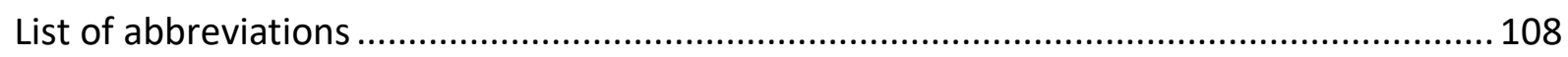

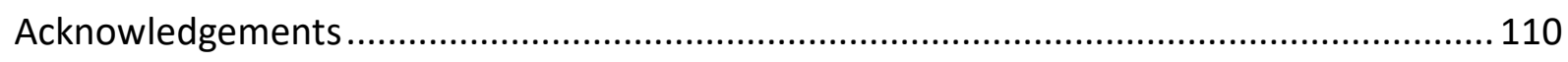

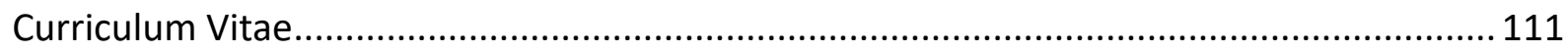

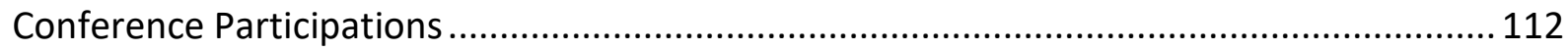




\section{Abstract}

Influenza A virus (IAV) increases morbidity and mortality rates and novel antivirals are needed to combat the virus. Errors of the viral polymerase lead to the generation of defective RNAs. These DI-RNAs may interfere with wild-type (wt) IAV infection and may be packaged into defective interfering particles (DIPs), which exhibit antiviral activity. DIPs inhibit IAV infection by competing with wt IAV for cellular and viral resources required for genome replication (replication interference) and by inducing interferon (IFN). DI-244 is a prototypic DI-RNA derived from IAV genomic segment 1 that harbours a large central deletion, it exerts potent antiviral activity and is considered for the development as antiviral. However, it is unclear whether DI-244 inhibits IAV via replication interference and/or IFN induction. Moreover, there is no system available to produce DI-244 in the absence of wt IAV, which raises safety concerns. The goal of this thesis was to close these research gaps by engineering MDCK cells to express codon optimized PB2 (PB2opt).

The PB2 open reading frame is destroyed in DI-244 and this defect should be complemented by the PB2 provided in trans. Indeed, MDCK-PB2opt cells in absence of wt IAV were able to produce DI-244 merely from plasmids. The generated DI-244 exerted strong antiviral activity against H1N1 and H3N2 IAV, but not against a dissimilar virus (vesicular stomatitis virus (VSV)). Furthermore, MDCK-PB2opt cells were successfully used to quantify DI-244 infectivity and thus constituted a useful tool to study how DI-244 inhibits IAV infection. This research revealed that any deletion in IAV genomic segment 1 could convert it into a DI-RNA and the antiviral activity was inversely correlated with DI-RNA length in the absence of a functional IFN system. In the presence of a functional IFN system, DI-244 induced a robust, partially STAT1-independent anti-IAV activity that was not determined by DI-RNA length and was more potent than DIP-mediated replication interference. Interestingly, RNAseq analysis and quantitative RT-PCR revealed that DI-244 induced expression of IFN-stimulating genes (ISGs) but not IFN, suggesting that DIPs might stimulate ISG expression via a novel pathway. In summary, the present study reports a system that allows production of DIPs in the absence of wt IAV and provides evidence that induction of the IFN system is a major contributor to DIP antiviral activity. Though, the induction of the IFN system does not involve DIPstimulated expression of IFN but direct induction of ISG expression. 


\section{Zusammenfassung}

Die Infektion mit dem Influenza-A-Virus (IAV) ist für hohe Morbidität und Mortalität verantwortlich und neue antivirale Medikamente werden dringend benötigt. Fehler der viralen Polymerase führen zur Bildung von defekten RNAs. Diese RNAs können mit der wt IAV-Infektion interferieren und in defective interfering particles (DIPs) verpackt werden, die antivirale Aktivität aufweisen. DIPs hemmen die IAV-Infektion indem sie mit wt IAV um Ressourcen konkurrieren, die für die Genomreplikation benötigt werden (Replikationsinterferenz), und indem sie das Interferon (IFN)-System aktivieren. DI-244 ist eine prototypische DI-RNA, die sich von dem genomischen Segment 1 von IAV ableitet und eine zentrale Deletion aufweist. DI-244 wirkt stark antiviral und könnte die Basis für ein neues Medikament darstellen. Es ist jedoch unklar, ob DI-244 die IAV-Infektion durch Replikationsinterferenz und/oder IFN-Induktion hemmt. Darüber kann DI-244 nicht ohne wt IAV hergestellt werden, was Sicherheitsbedenken aufwirft. Das Ziel dieser Arbeit war es, diese Forschungslücken zu schließen.

Für die Produktion von DI-244 ohne IAV wurden MDCK-Zellen hergestellt, die kodonoptimiertes PB2 (PB2opt) exprimieren. Das PB2-Leseraster in DI-244 ist zerstört und dieser Defekt sollte durch das in trans bereitgestellte PB2 komplementiert werden. Tatsächlich gelang es DI-244 in MDCK-PB2opt Zellen mit Hilfe von Plasmiden und ohne wt IAV herzustellen. Das so erzeugte DI-244 hemmte H1N1- und H3N2-IAV und die DIPInfektiosität konnte mit Hilfe der MDCK-PB2opt-Zellen quantifiziert werden. Mechanistische Analysen zeigten, dass jede Deletion im IAV-Genomsegment 1 dieses in eine DI-RNA verwandelte, deren antivirale Aktivität in Abwesenheit eines funktionierenden IFN-Systems invers mit der Länge der DI-RNA korrelierte. In IFN-kompetenten Zellen induzierte DI-244 eine robuste, teilweise STAT1-unabhängige anti-IAV-Aktivität, die nicht durch die DI-RNALänge bestimmt wurde und die stärker war als die DIP-vermittelte Replikationsinterferenz. Interessanterweise zeigten RNAseq- und PCR-Analysen, dass DI-244 die Expression von IFNstimulierten Genen (ISGs), aber nicht von IFN induzierte, was darauf hindeutet, dass DIPs die ISG-Expression über einen neuartigen Weg stimulieren können. Zusammenfassend zeigt diese Arbeit, dass DIPs in Abwesenheit von wt IAV hergestellt werden können und IFNunabhängig die ISG-Expression induzieren, was wahrscheinlich wesentlich zu ihrer antiviralen Aktivität beiträgt. 


\section{Introduction}

\subsection{Viruses}

Viruses are intracellular parasites which require host cells for their replication. They harbour genetic material (DNA or RNA) that is protected by a protein shell and, for some viruses, a membrane. Viruses enter host cells by binding to surface receptors and hijack the biosynthesis machinery of the cell for gene expression and genome replication. Mutations occurring during genome replication can provide viruses with new biological properties, for instance, the ability to infect new hosts or to evade the immune response. Infection with well-studied viruses like human immunodeficiency virus (HIV) and influenza viruses is responsible for considerable global morbidity and mortality. Moreover, the emergence of new, highly transmissible viruses can threaten human health and economies, as evidenced by the current COVID-19 pandemic, which is caused by a novel coronavirus, the severe acute respiratory syndrome coronavirus 2 (SARS-CoV-2).

\subsubsection{Influenza Viruses}

Influenza viruses cause acute infections of the respiratory tract. Symptoms usually last up to 3-7 days and include sore throat, cough, fever, running nose, chills, and muscle aches (Ohmit and Monto 2006; Monto et al. 2000; Public Health England 2019). Influenza virus infection can also cause severe illness or death, especially in people with comorbidities, pregnant women, individuals with compromised immune system (Ramsay 2019) and infants aged 6 months or younger (Cromer et al. 2014). Influenza viruses spread via droplets released by an infected person through sneezing and coughing, but also by contact with contaminated surfaces (Lau et al. 2010). The World Health Organisation (WHO) estimates that 3-5 million cases of severe illness and 0.3-0.5 million deaths occur annually worldwide due to recurring seasonal influenza (WHO 2020b). Furthermore, influenza pandemics may result in millions of deaths as seen with the so-called Spanish influenza in 1918.

Influenza viruses belong to the family Orthomyxoviridae and are enveloped viruses with a segmented, negative-stranded RNA genome (Shaw and Palese 2013). They are divided into 4 different genera: influenza A, B, C and D viruses (WHO 2020a, 2020c; Ghebrehewet et al. 2016). Influenza A viruses (IAV) are further classified into subtypes according to their hemagglutinin (HA) and neuraminidase (NA) proteins and at present 18 different subtypes of HA and 11 subtypes of NA are known (Paules and Subbarao 2017). Two additional HA (H17 
and $\mathrm{H} 18$ ) and NA (N10 and N11) subtypes have recently been identified in bats (Tong et al. 2012; Tong et al. 2013). Influenza B viruses (IBV) are classified into 2 lineages - Victoria and Yamagata (Bennett et al. 2015; WHO 2020a, 2020c). Influenza virus strains are named according to their genus (type), the species from which the virus was isolated, the geographical location where the isolate was obtained, the number of the isolate, and the year of isolation (Shaw and Palese 2013). For example, A/Puerto Rico/8/34 (H1N1) designates $8^{\text {th }}$ isolate of an IAV strain that was isolated from a human patient in Puerto Rico in 1934 and has antigenic subtypes 1 for both HA and NA (Krug 1989). A/Puerto Rico/8/34 hereafter will be referred to as PR8. Influenza A, B and C viruses are known to infect humans (Webster et al. 1992) while influenza D viruses mainly infect pigs and cattle (Hause et al. 2014; Ferguson et al. 2015). Both IAV and IBV can induce severe influenza but only IAV causes both epidemics and pandemics. Influenza C viruses cause only mild illness (Matsuzaki et al. 2016) and are clinically not relevant.

Influenza viruses are spherical or filamentous in form with an average diameter of $100 \mathrm{~nm}$ and a length of $300 \mathrm{~nm}$ (Bouvier and Palese 2008). Particles contain an envelope, the viral genome, and viral proteins. A schematic diagram of the IAV structure is depicted in Figure 3.1. Its envelope is formed by a lipid bilayer that harbours three viral proteins, HA, NA and the ion channel (M2). A layer of matrix protein (M1) is located below the membrane and the particle interior contains eight segments of genomic viral RNA (VRNA) associated with viral proteins in a ribonucleoprotein (vRNP) complex (Bouvier and Palese 2008).

Each of the eight VRNP contains VRNA associated with nucleoprotein (NP) and the viral RNAdependent RNA polymerase (RdRp), which consists of the subunits polymerase basic 1 (PB1) and 2 (PB2) and polymerase acidic (PA) (Nogales and Martínez-Sobrido 2016). The coding region of each vRNA is flanked by non-coding regions (NCR) which are conserved for each segment among all IAV strains. The NCRs act as promoters to initiate RNA replication and mRNA expression. Sequences adjacent to the NCRs are segment-specific and together with the NCRs form the packaging signals required for the incorporation of VRNPs into progeny viral particles as well as polyadenylation signals. The eight segments vary in length with the segment encoding PB2 being the longest and the segment encoding the non-structural (NS) proteins being the shortest. Each viral segment encodes one or more viral proteins with specific functions (Table 3.1) (Bouvier and Palese 2008). 


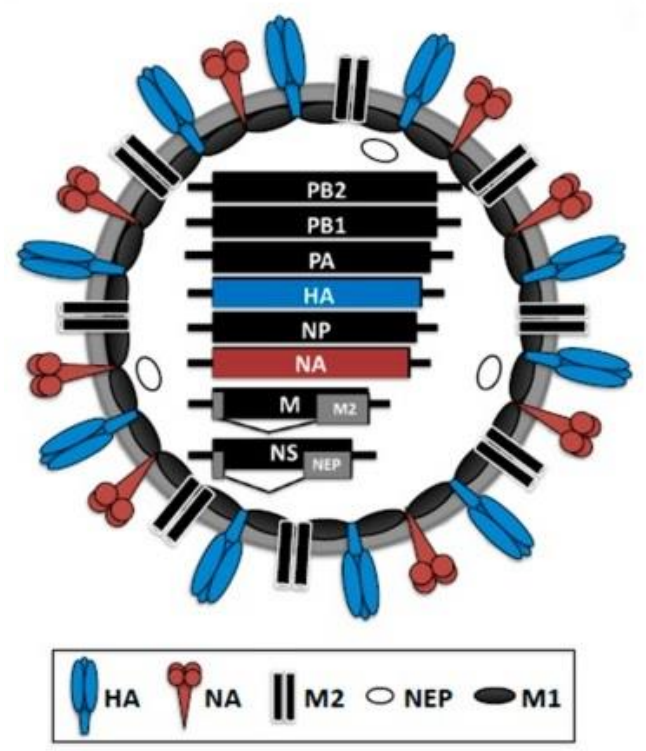

Figure 3.1 A schematic diagram of influenza A virus particle structure. The HA, NA, and matrix (M2) proteins are inserted into the lipid bilayer. Matrix protein (M1) surrounds the nucleocapsid, which compromises eight ssRNA segments associated with nucleoprotein (NP). The eight RNA segments encode 10 proteins: 3 polymerase subunits (PB2, PB1, PA), HA, NP, NA, M1, M2, NS1, and NEP. Black lines in the vRNA segments represent $3^{\prime}$ and $5^{\prime}$ non-coding regions (NCR). PB1 and PB2, polymerase basic 1 and 2; PA, polymerase acidic; NP, nucleoprotein; M, Matrix; NS, non-structural; NEP, nuclear export protein. [Taken from (Nogales and Martínez-Sobrido 2016)]

Table 3.1 Length and function of genome segments of influenza A virus (Bouvier and Palese 2008) *(Hayashi et al. 2015) nt, nucleotides

\begin{tabular}{|c|c|c|c|c|}
\hline Segment & $\begin{array}{l}\text { Length } \\
\text { (nt) }\end{array}$ & Proteins & & Function \\
\hline 1 & 2341 & PB2 & Polymerase basic 2 & Cap recognition \\
\hline \multirow[t]{3}{*}{2} & 2341 & PB1 & Polymerase basic 1 & Elongation \\
\hline & & PB1-F2 & $\begin{array}{l}\text { (Open reading frame } \\
\text { overlapping with }\end{array}$ & $\begin{array}{l}\text { Pro-apoptotic activity, Interferon } \\
\text { antagonist }\end{array}$ \\
\hline & & PB1-N40 & PB1) & Unknown \\
\hline \multirow[t]{2}{*}{3} & 2233 & PA & Polymerase acidic & Endonuclease activity, protease \\
\hline & & $P A-X$ & & Host shut-off* \\
\hline 4 & 1778 & $\mathrm{HA}$ & Hemagglutinin & $\begin{array}{l}\text { Major antigen, receptor binding, } \\
\text { membrane fusion }\end{array}$ \\
\hline 5 & 1565 & NP & Nucleoprotein & RNA binding, RNP nuclear export \\
\hline 6 & 1413 & NA & Neuraminidase & Sialidase activity, virus release \\
\hline \multirow[t]{2}{*}{7} & 1027 & M1 & Matrix protein 1 & $\begin{array}{l}\text { VRNP and glycoprotein interaction, } \\
\text { RNP nuclear export, assembly and } \\
\text { budding }\end{array}$ \\
\hline & & M2 & Matrix protein 2 & $\begin{array}{l}\text { Ion channel activity, virus uncoating } \\
\text { and assembly }\end{array}$ \\
\hline \multirow[t]{2}{*}{8} & 890 & NS1 & $\begin{array}{l}\text { Non-structural } \\
\text { protein } 1\end{array}$ & Interferon antagonist activity \\
\hline & & $\begin{array}{l}\text { NS2 } \\
\text { /NEP }\end{array}$ & $\begin{array}{l}\text { Non-structural } \\
\text { protein } 2 / \text { Nuclear } \\
\text { Export protein }\end{array}$ & Regulation of RNA synthesis \\
\hline
\end{tabular}




\subsubsection{Biology of influenza A viruses}

Influenza A viruses (IAVs) infect diverse mammals, including humans, pigs, dogs, cats, and birds (Webster et al. 1992; Hussain et al. 2017). The natural reservoir host of IAVs are wild aquatic birds (Webster et al. 1992). The interspecies transmission of IAVs and the constant adaptation of these viruses to immune pressure promotes antigenic variation in HA and NA (Neumann et al. 2009). Thus, the constant acquisition of point mutations in NA and particularly in HA during the circulation of IAV and IBV in humans allow these viruses to evade antibody-mediated neutralization and to continuously spread in immunologically nonnaïve human populations. This process is termed antigenic drift (Paules and Subbarao 2017) and the mutations arise due to the absence of a proof-reading activity of the viral RNAdependant RNA polymerase (RdRp) (Cox and Subbarao 2000; Bennett et al. 2015). Antigenic drift is responsible for seasonal epidemics and for the constant need to reformulate vaccines (Paules and Subbarao 2017). Novel IAV can arise when two or more IAV coinfect cells and exchange genomic segments, which is termed reassortment. If these IAV harbour antigenically novel HA and NA proteins, i.e. proteins against which humans have not preexisting immunity, this process is called antigenic shift. It may result in IAVs that can rapidly spread in the human population and lead to influenza pandemics (Paules and Subbarao 2017; Ramsay 2019) .

The deadliest known pandemic was the so-called Spanish influenza (Spanish flu) which occurred in 1918. The responsible H1N1 virus was the result of a reassortment of a human $\mathrm{H} 1$ and an avian N1 subtype virus and caused over 50 million deaths worldwide (Frost 1919; Neumann et al. 2009; CDC 2019a). The next IAV pandemic occurred in 1957 and is termed Asian flu. It was caused by an H2N2 virus, which emerged due to reassortment of the then circulating seasonal H1N1 IAV with an avian IAV, and resulted in 1-2 million deaths (Neumann et al. 2009; CDC 2019b). The next influenza pandemic was recorded in 1968 and is termed Hong Kong flu. It was caused by an H3N2 virus that emerged due to reassortment between the then circulating seasonal virus of the H2N2 subtype and an avian H3 subtype (Neumann et al. 2009). About 1 million deaths are attributed to the Hong Kong flu (CDC 2019c). The most recent influenza pandemic was the 2009 Swine flu. The responsible virus originated from reassortments between human H3N2, North American avian and H1N1 swine viruses followed by a further reassortment with an Eurasian avian-like swine H1N1 
virus (Dawood et al. 2009; Neumann et al. 2009). The total number of deaths attributed to swine flu was 150,000-575,000 (CDC 2019d) and the consequences of the swine flu were thus comparable to that of seasonal influenza.

In the past decades, IAV subtypes such as H5N1, H7N9, and H10N8 originating from birds were transmitted to humans, but human infection frequently requires close contact and exposure to high amounts of virus, which limits the transmissibility of these viruses (Lu et al. 2016).

\subsubsection{Replication Cycle}

The replication cycle of IAV begins with the binding of the viral HA to cell surface proteins and lipids modified with sialic acids (Dou et al. 2018), where the HA of human and avian IAV subtype attaches to $\alpha-2,6$-linkages and $\alpha-2,3-$ linkages, respectively (Bouvier and Palese 2008). Following attachment, the virus is taken up into endosomal compartment, where the low $\mathrm{pH}$ environment stimulates the next steps required for infection. First, it initiates a conformational change in $\mathrm{HA}$ and that results in fusion between the viral and the endosomal membrane (Dou et al. 2018). Membrane fusion requires cleavage of HA by the cellular serine protease TMPRSS2 (transmembrane protease serine S1 member 2) in infected cells (Böttcher et al. 2006) or addition of trypsin to the culture medium (Klenk et al. 1975). The only exception is A/Wilson-Smith Neurotropic/33 (A/WSN/33) for which NA ensures HA cleavage by recruiting plasminogen (Goto and Kawaoka 1998; Goto et al. 2001; Chaipan et al. 2009). A/WSN/33 hereafter will be referred to as WSN.

Second, the hydrogen ions are transported via the $M 2$ ion channel into the interior of virus particles, which facilitates disassembly by abrogating protein-protein interactions (Shaw and Palese 2013). This allows release of viral ribo-nucleoprotein (VRNPs) particles into the cytoplasm and subsequent transport to the nucleus (Dou et al. 2018). In the nucleus, the viral polymerase, consisting of PB1, PB2 and PA transcribes the VRNA into 5' capped and 3' polyadenylated mRNAs. For this, the viral polymerase cuts cellular mRNAs near the $5^{\prime}$ end and uses the resulting small RNA segments as primers for transcription, a process termed cap snatching. Viral mRNA is then exported to the cytoplasm for translation of viral proteins ( $H A$, NA and M2). Moreover, the viral polymerase generates complementary RNA (cRNA), which serves as a template for production of vRNA (Figure 3.2). Newly synthesized vRNPs are 
transported into cytoplasm by M1 and NS2/NEP where assembly of progeny virions occurs thereafter released by budding from the plasma membrane (Te Velthuis and Fodor 2016).

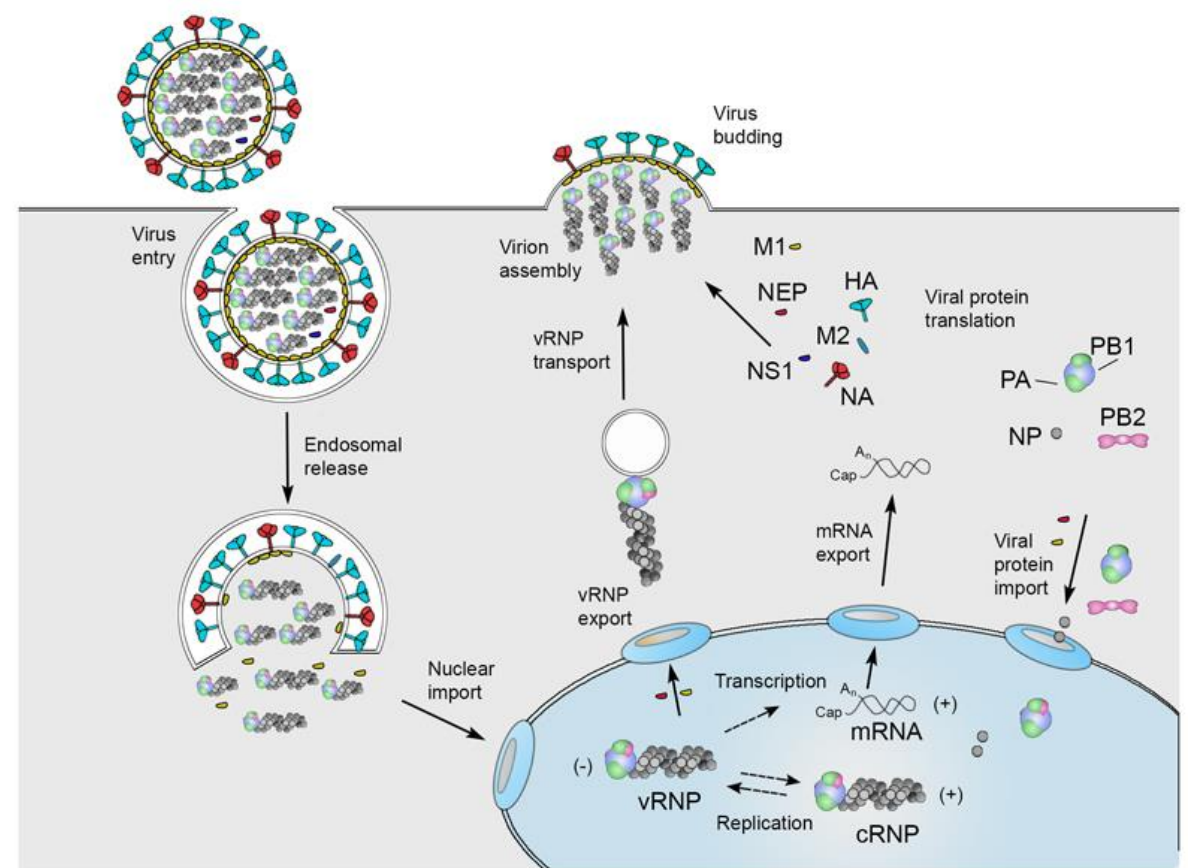

Figure 3.2 Influenza A virus replication cycle. The virus is engulfed after binding to cell surface receptors followed by endocytosis. After fusion of endosomal and viral membrane vRNPs are released into the cytoplasm. Subsequently, they are imported to the nucleus for viral replication and transcription. Thereafter, viral mRNA is exported for the translation of viral proteins to the cytoplasm. Finally, virus assembles and buds from the cell surface (Te Velthuis and Fodor 2016).

\subsubsection{Reverse genetics system of influenza $A$ virus}

The generation and modification of recombinant influenza viruses require reverse genetics systems. The first reverse genetics system was established in 1999 using twelve plasmids: four expression plasmids for the viral RdRp complex and NP for reconstitution of VRNP and eight RNA polymerase-I (Pol-I) driven plasmids for transcription of VRNA segments (Neumann et al. 1999). Later the use of bidirectional plasmids allowed complete reconstitution of influenza virus from only 8 plasmids (Hoffmann et al. 2000a; Hoffmann et al. 2000b; Hoffmann 2002) (Figure 3.3 A). The highlight of this system is that each plasmid contains RNA Pol-I and-II promoters in opposite directions which, using the same cDNA, drive the expression of VRNA and encoded protein(s), respectively (Figure 3.3 A). Briefly, human Pol-I promoter and mouse Pol-I terminator are used to synthesize influenza vRNAs. Pol-I transcribes VRNA which is recognised by the viral polymerase complex. A Pol-II dependent cytomegalovirus promoter and a polyadenylation sequence direct the synthesis of segmented proteins from the same viral cDNAs (Figure 3.3 A). Based on this system 
recombinant influenza viruses harbouring reporter genes were constructed and used to study virus replication and spread within the cell culture and in mice (Manicassamy et al. 2010; Eckert et al. 2014; Nogales et al. 2015; Breen et al. 2016).

The generation of recombinant influenza virus involves transfection of eight bi-directional plasmids into a co-culture of 293T and Madin-Darby Canine Kidney (MDCK) cells, where 293T cells provide high transfection efficiency while MDCK cells are known to provide high infectious titers (Martínez-Sobrido and García-Sastre 2010). During transfection of cells with these plasmids, Pol-I generates the eight-negative sense vRNAs while Pol-II directs the synthesis of viral proteins which are translated from viral mRNAs. Following translation, NP and polymerase complex associate with vRNAs to form VRNP complex (Figure 3.3 B) (Nogales and Martínez-Sobrido 2016). Consequently, the replication cycle process is initiated (section 3.1.1.2).

A)

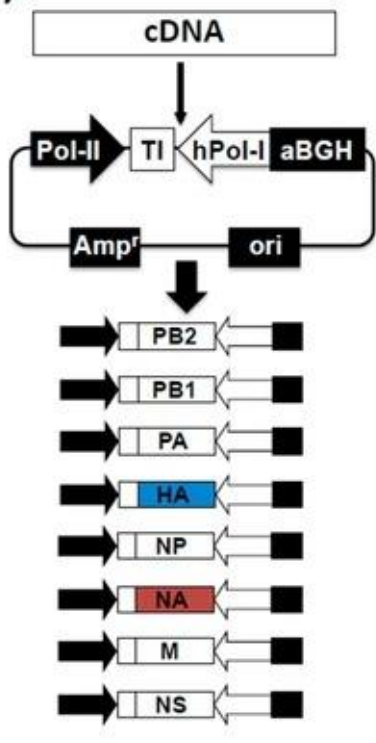

B)

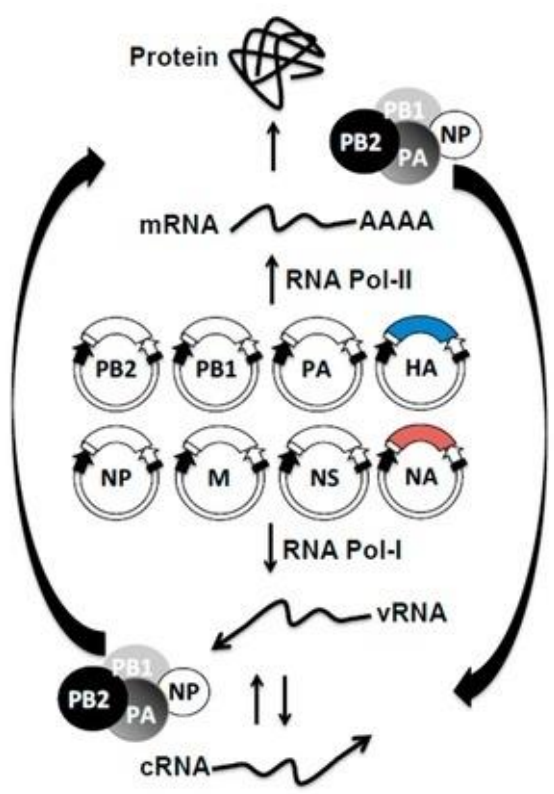

Figure 3.3 Influenza vRNA cloning and reverse genetics A) VRNA cloning into ambisense plasmid: a bidirectional vector containing hPol-I and T1 to direct the synthesis of the vRNAs. In opposite orientation to Pol-I cassette, a Pol-II and aBGH directs the synthesis of proteins from same viral cDNAs hPol-I, human polymerase-I promoter; T1, mouse pol-I terminator; Pol-II, polymerase-II cytomegalovirus promoter; aBGH, polyadenylation signal of the gene encoding bovine growth hormone; Ori, plasmid origin of replication; Ampr, Ampicillin resistance gene $B$ ) In cells transfected with ambisense plasmids, the Pol-I cassette generates 8 (-) vRNAs while the Pol-II directs the synthesis of 8 viral mRNAs that are translated into viral proteins. (Nogales and MartínezSobrido 2016)

\subsection{Prophylaxis and Therapy}

Infants, immunocompromised patients and the elderly are at risk of developing severe or fatal influenza (Mallia and Johnston 2007; Troeger et al. 2019), vaccination provides the 
most effective protection (Houser and Subbarao 2015). However, vaccines against seasonal influenza will offer little to no protection against pandemic influenza. Besides vaccines, antiinfluenza drugs targeting viral proteins are available to combat influenza. Though, they have certain shortcomings such as high resistance, no activity against IBV and associated with side effects (Li et al. 2018).

\subsubsection{Vaccines}

Anti-IAV vaccines need to be reformulated on an annual basis due to antigenic drift. The current seasonal influenza vaccines are trivalent (H1N1, H3N2, one strain of IBV) or quadrivalent (H1N1, H3N2, two strains of IBV) (WHO 2020c; Grohskopf et al. 2019). Three classes of licenced vaccines are available. Inactivated influenza vaccines (IIV) contain noninfectious particles and are commonly used. Live attenuated influenza vaccine (LAIV) contains a mixture of four strains of infectious influenza viruses with temperature-sensitive and attenuating mutations (Coelingh et al. 2014). Recombinant influenza vaccine (RIV) uses the baculovirus expression system or other viral vectors for production of recombinant $\mathrm{HA}$ (Grohskopf et al. 2019)

Influenza vaccines are frequently generated in embryonated chicken eggs. The production involves isolation of selected strains, virus propagation in the allantoic cavity of eggs, harvesting, inactivation, purification and concentration (Stöhr et al. 2012). Despite being a well-established process, the whole vaccine production is time-consuming with several drawbacks such as limited availability of eggs, allergic reactions to residual egg protein and adaptation of influenza viruses to spread in eggs (Houser and Subbarao 2015). In the past decade, cell-based influenza vaccines were developed and are available for human treatment (Gallo-Ramírez et al. 2015). They are produced in three different cell lines: MDCK cells, African monkey kidney epithelial cells (Vero) or primary cultures of human retinoblasts (PER.C6) (Milián and Kamen 2015). The use of cell-based vaccines avoids allergic reactions to individuals sensitive to egg proteins, lowers the risk of contamination of eggs as a substrate, and provides easier and less expensive purification of the desired antigen and high scalability (Rappuoli 2006; Hegde 2015). However, despite the advancement in cell-culture vaccines, there is no universal flu vaccine. 


\subsubsection{Anti-influenza drugs}

Different classes of antiviral drugs have been approved for influenza treatment and the drugs target different steps of the viral replication cycle.

The first class of antivirals approved are the adamantanes (Hayden et al. 1980), amantadine and rimantadine, which block the M2 ion channel and thereby inhibit viral disassembly (Hay et al. 1985). However, viruses rapidly acquire mutations in $M 2$ that causes drug resistance (Hussain et al. 2017; Han et al. 2018). Therefore, use of M2 inhibitors is not recommended.

The second class are NA inhibitors: oseltamivir, zanamivir, peramivir and laninamivir inhibit NA activity and prevent viral spread (Hussain et al. 2017; Han et al. 2018). Nevertheless, viruses can acquire resistance conferring mutations, particularly against oseltamivir. For example, a mutation occurred in the NA of 2009 pandemic H1N1 virus strain, resulting in the inactivity of the oseltamivir drug (Morlighem et al. 2011).

The third class of inhibitors is represented by Arbidol, which inhibits HA-driven membrane fusion. This drug is licenced in Russia and China for treatment of seasonal influenza (Paules and Subbarao 2017).

The fourth class of inhibitor is the RNA-dependent RNA polymerase inhibitor Favipiravir. It inhibits the RdRp of influenza virus preventing vRNA replication and transcription (Jin et al. 2013). It is approved only in Japan and its use is highly restricted (Ison 2015).

Fifth is Baloxavir Marboxil, an enzyme inhibitor targeting IAV cap-snatching by the viral PA subunit. It inhibits the cap-snatching process, thus blocking viral translation (Hayden et al. 2018).

Nevertheless, both IAV and IBV can become resistant to these drugs spontaneously or during antiviral treatment and some drugs have no activity against IBV. Therefore, novel antiviral approaches are needed and DIPs are one option.

\subsection{Defective Interfering Particles (DIPs)}

DIPs are virus mutants that naturally appears when a critical fragment of the viral genome is deleted completely or to an extent of non-functionality due to a replication error (Marriott and Dimmock 2010). DIPs were defined based on the following criteria 1) they are produced and amplified at high multiplicity of infection (MOI) 2) they are replication-deficient and need wild-type (wt) virus for their spread 3 ) they interfere with wt virus infection, when cells 
are co-infected by DIPs and wt virus, DIPs are produced at the expense of wt virus 4) they contain a shortened version of the wt virus genome 5) they are responsible for interference (Nayak et al. 1978; Nayak et al. 1985). Any RNA possessing interfering ability is termed defective interfering RNA (DI-RNA) and the virus which helps DI-RNA replicate by providing the missing viral protein is termed as wt virus (Nayak et al. 1985).

\subsubsection{Overview of the types of DI-RNAs}

DIPs are known to be produced during DNA and RNA virus infection (Perrault 1981) in the laboratory and in the host. Viruses for which DIP formation has been described include the RNA viruses - Dengue virus, Polio virus, VSV, Semliki forest virus, SARS coronavirus, West Nile virus, Influenza virus, the DNA virus - Herpes simplex virus and plant viruses like Geminiviruses (Yang et al. 2019). The term DIPs was first proposed in 1970 (Huang and Baltimore 1970), although Von Magnus first described particle preparations with inhibitory activity as incomplete influenza virus particles in 1950 (Magnus 1954).

The generation and amplification of DI-RNAs are autonomous processes. The generation of DI-RNAs depends on transcription of viral RNA during a high MOl infection (Nayak et al. 1978) and two mechanisms for generation of DI-RNAs were postulated. The "jumping polymerase" mechanism encompasses the polymerase detaching from one segment or a part of the template to another and reattaching to give rise to short mRNA strand ensuring to synthesize this mRNA before it further springs. Alternatively, in the "rolling polymerase" mechanism the polymerase does not completely separate from the template, instead it rolls over to a new site of the template located nearby (Nayak et al. 1985; Nayak et al. 1982). The range of genome present in DIPs are listed in Table 3.2 and depicted in Figure 3.4.

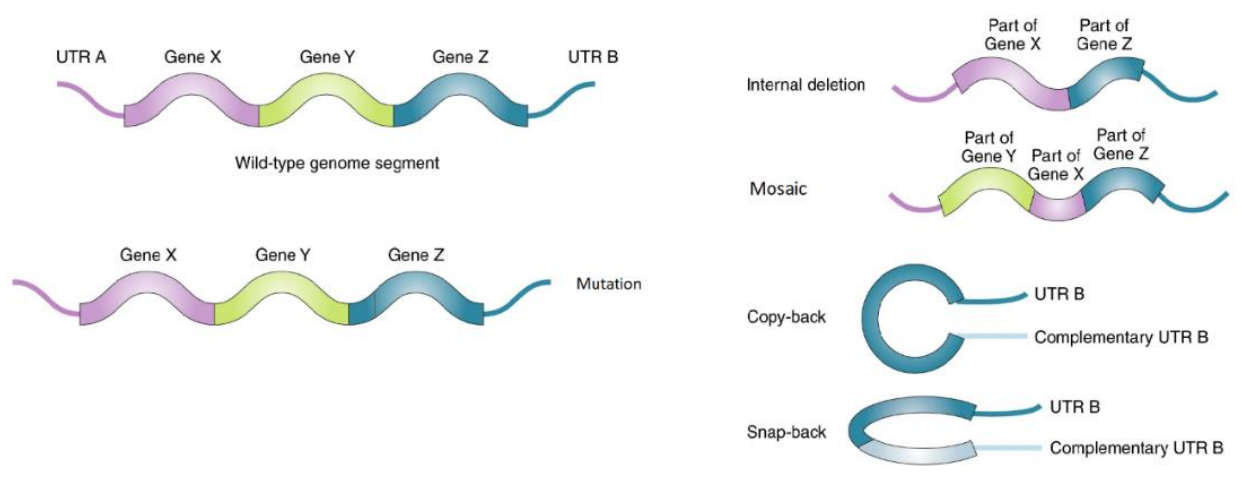

Figure 3.4 Types of defective interfering RNAs [adapted from (Vignuzzi and López 2019)] UTR: Untranslated region present one on each side of a coding sequence on a strand of RNA 
Table 3.2 Types of defective interfering RNA (DI-RNA) (Kolakofsky 1976; Leppert 1977):

\begin{tabular}{|c|c|c|c|}
\hline Types of DI-RNA & DI-RNA arises when & Example & References \\
\hline $\begin{array}{l}\text { Simple internal } \\
\text { deletion }\end{array}$ & $\begin{array}{l}\text { part of the genome is deleted } \\
\text { or skipped during replication }\end{array}$ & $\begin{array}{l}\text { Influenza A virus; } \\
\text { Flock house virus }\end{array}$ & $\begin{array}{c}\text { (Perrault 1981; } \\
\text { Jaworski and Routh } \\
\text { 2017) }\end{array}$ \\
\hline $\begin{array}{l}\text { Snap back or } \\
\text { hairpin }\end{array}$ & $\begin{array}{l}\text { replicase transcribes part of } \\
\text { the genome, which snaps back } \\
\text { and is then used as a template }\end{array}$ & $\begin{array}{l}\text { Vesicular } \\
\text { stomatitis virus }\end{array}$ & $\begin{array}{l}\text { (Schubert and } \\
\text { Lazzarini 1981) }\end{array}$ \\
\hline $\begin{array}{l}\text { Copy back or } \\
\text { Panhandle }\end{array}$ & $\begin{array}{l}\text { polymerase carries a partially } \\
\text { made strand and switches } \\
\text { back to transcribe } 5^{\prime} \text { termini }\end{array}$ & $\begin{array}{l}\text { Human } \\
\text { metapneumovirus; } \\
\text { Measles virus }\end{array}$ & $\begin{array}{l}\text { (van den Hoogen, } \\
\text { Bernadette G. et al. } \\
\text { 2014; Mura et al. } \\
\text { 2017) }\end{array}$ \\
\hline Mosaic & $\begin{array}{l}\text { various regions may come } \\
\text { from same wt virus but in an } \\
\text { incorrect order }\end{array}$ & Tombusvirus & $\begin{array}{c}\text { (White and Morris } \\
\text { 1994) }\end{array}$ \\
\hline Mutation & $\begin{array}{l}\text { mutations arise in functionally } \\
\text { important parts of viral } \\
\text { genome }\end{array}$ & Influenza A virus & (Kupke et al. 2019) \\
\hline
\end{tabular}

\subsubsection{Origin of DI-RNA of IAV}

In influenza virus infection a minimum of three types of particles are formed: wt particles, defective interfering particles (DIPs) (Nayak 1980), and defective non-interfering particles (Hirst and Pons 1973). These particles cannot be substantially separated from each other and the ratio with which they are generated may vary between different virus preparations and can be determined by plaque assay since non-plaque formers are believed to be defective because of the lack of a complete virus genome (Nayak et al. 1985).

In 1954, IAV was serially passaged in embryonated chicken eggs at a high MOI. As a result, a significant decrease in infectivity relative to HA titer was observed. It was due to the formation of DIPs (Magnus 1954; Huang and Baltimore 1970). The interfering aspect of DIPs was already known from Von Magnus' studies who infected mice with a fourth undiluted PR8 passage and observed complete suppression of wt IAV growth (Magnus 1954). In another study the infectious virus load was reduced but provided no protection (Holland and Doyle 1973). Though, in general, the stability and level of protection was low, which led to inconclusive and unreliable results. To circumvent this issue, DI-RNA sequences were cloned with DIPs and wt virus jointly generated by reverse genetics approaches (Duhaut and Dimmock 2002). The most extensively studied DI-RNA is DI-244 that arose 
extemporaneously in PR8 infected cells and was cloned using recombinant virus technology. It has a large central deletion in segment 1 of IAV which encodes for the PB2 protein. Despite the large deletion the terminal sequences required for genome packaging remain intact in DI-244. DI-244 RNA has a total length of 395 nucleotides (nt) and has 244 nt at the 3' end and $151 \mathrm{nt}$ at the $5^{\prime}$ end, as depicted in Figure 3.5 (Dimmock et al. 2008).

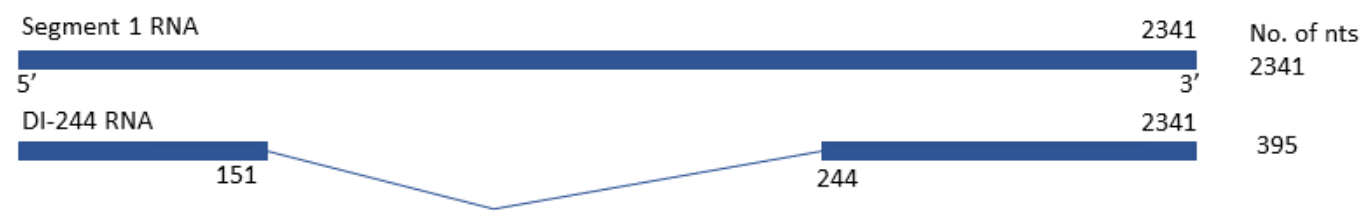

Figure 3.5 Schematic diagram of DI-244. DI-244 was derived from segment 1 of PR8, which encodes for PB2 protein. The name stems from the $244 \mathrm{nt}$ remaining at the $3^{\prime}$ end. The total number of nucleotides is shown at the right. The nucleotides remaining after central deletion are indicated at the breakpoints [adapted from (Meng et al. 2017)].

For DI-244 production, eight wt plasmids and DI-244 encoding plasmid are cotransfected into 293 T cells and supernatants passaged in embryonated chicken eggs (Dimmock et al. 2008) followed by purification through sucrose gradient centrifugation (Nayak 1980). This mixture is treated with UV-irradiation to inactivate wt IAV (Dimmock and Easton 2015) since physical separation of DI-RNA and wt virus genome is not possible due to similar particle size and density (Nayak et al. 1985). During UV inactivation, wt virus infectivity is selectively eliminated due to large size differences: $395 \mathrm{nt}$ for DI-244 as compared to 13,600 nt for wtIAV (Dimmock et al. 2008). Long duration of UV-inactivation (about 8 minutes) inactivates all its protecting activity and infectivity, called inactive DI-244 (Dimmock et al. 2008).

\subsubsection{Application of DIPs as antivirals}

Dimmock and his colleagues showed that DI-244 DIP preparations are active both prophylactically and therapeutically (Dimmock et al. 2012a; Easton et al. 2011; Scott et al. 2011c, 2011a). For their studies they used DI-244 produced via reverse genetics (Dimmock et al. 2008). To demonstrate homologous protection, they admisnistered DI-244 (DIPs) to mice jointly with WSN or first infected the animals with IAV and than administered DI-244. The mice were protected in both settings but protection decreased when the animals were 
infected with virus 4 days before administration of DIPs and was completely lost when viruses was added 7 days before DIPs (Dimmock and Easton 2014; Dimmock et al. 2008).

The ratio of DIP to wt virus plays an crucial role in DIP anti-viral activity. As infection progresses, the number of infectious wt IAV particles increases significantly where the amount of DIPs administered becomes insufficient to influence the infection. A concern from the preclinical studies of DI-244 was that replication of wt virus was reduced but not completely repressed. Ferrets are important animal models of IAV infection, and are used to evaluate vaccines. In a ferret study, animals were protected from infection with the pandemic A/California/04/2009 virus when DI-244 was given 2 hours prior to wt IAV infection and were immune to reinfection with A/Cal (Dimmock et al. 2012b).

Besides protection against homologous viruses, DI-244 was shown to protect in vivo against heterologous viruses such as B/Lee/40 (IBV) (Scott et al. 2011a) and pneumonia virus of mice (PVM) (Easton et al. 2011). In these studies, coadministration of DI-244 with IBV protected the animals from clinical disease and pre-treatment of mice with DI-244 augmented the efficiency of protection (Scott et al. 2011a). Similarly, pre-treatment with a single dose of DI244 protected mice from PVM (Easton et al. 2011). A comparative study of heterologous protection from PVM and homologous protection from IAV showed that homologous protection was 5 -fold more effective. This difference could be due to different mechanisms underlying the two types of protection (Dimmock and Easton 2014), which is summarized in below Figure 3.6. The mechanisms are defined later in detail.

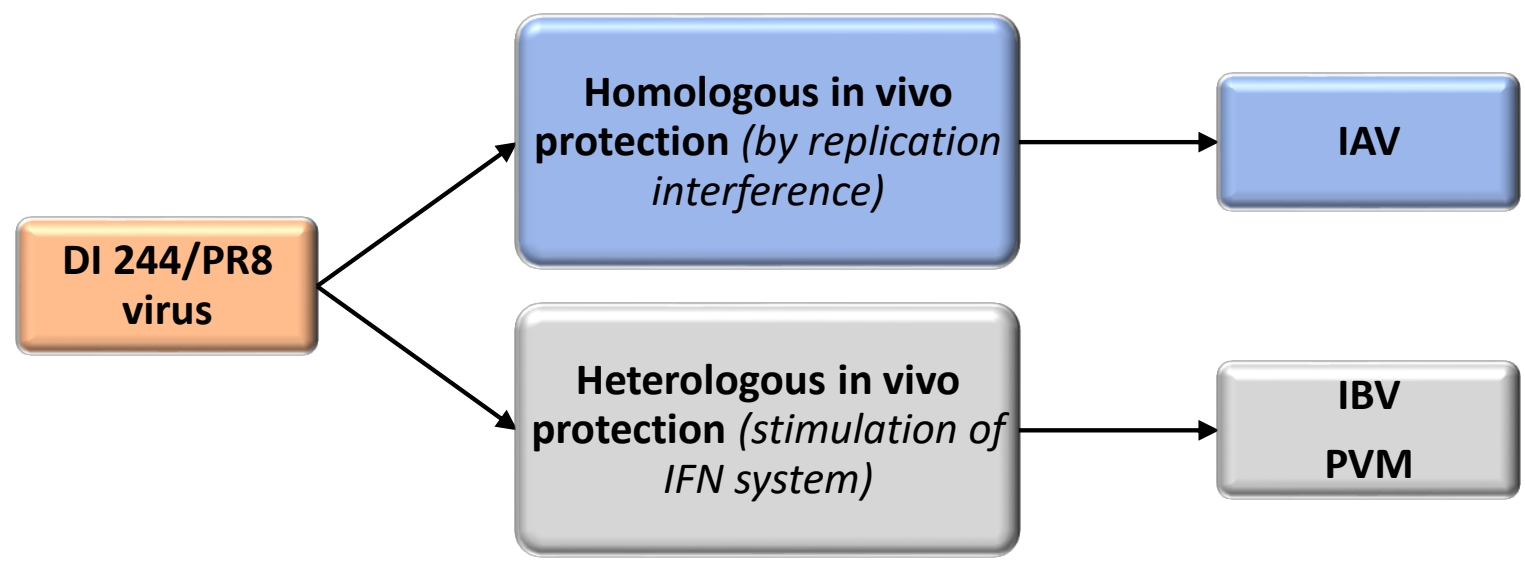

Figure 3.6 Overview of homologous and heterologous protection. DI-244 RNA protects from homologous challenge with IAV by replication interference and stimulation of the IFN system while protection against heterologous challenge is solely due to stimulation of the IFN system. [Adapted from (Easton et al. 2011)] 
These studies prove, that $\mathrm{DI}-244$ 1) converts a potentially lethal infection into an avirulent infection 2) protection is possible during a substantial time window with a lower concentration of DIPs 3 ) is more effective than a non-clonal DI virus in providing protection (Dimmock and Easton 2014). However, these studies also demonstrated that DI viruses 1) are ineffective and could be diluted when not administered systematically targeting specific areas of organ (Dimmock and Easton 2015) 2) comprised of complex mixtures of different DI-RNAs (Jennings et al. 1983; Duhaut and Dimmock 1998) 3) unavoidably contained mixtures of defective RNAs that did not interfere with the replication of wt virus (Barrett et al. 1984). 4) heterogeneity led to reproducibility issues and prevented that solid conclusions on the potential of DIPs as antiviral could be drawn (Dimmock and Easton 2014).

Wasik and colleagues reported an approach to reduce genetic variablity of DIPs (Wasik et al. 2018). DI-244 was replicated in a designer cell line, AGE1.CR.pIX, originated from Muscovy duck and known to propagate influenza viruses (Lohr et al. 2009) faster than primary chicken cells (Jordan et al. 2009). The wt virus used to amplify DIPs was characterized to be free of deletions in other segments. This approach allowed DIP production under well-controlled conditions but still relied on the use wt IAV (Wasik et al. 2018). This limitation could be addressed by generating cell lines that provide these viral proteins in trans that are not encoded by DIPs because the respective open reading frames were destroyed during DI-RNA generation.

\subsubsection{Antiviral mechanism of DIPs}

Several mechanisms have been proposed for the inhibition of influenza virus infection by DIPs including replication interference, IFN induction, viral persistence and virulence modulation (Vignuzzi and López 2019) Figure 3.7. Replication interference and IFN induction will be discussed below. Viral persistence, the mechanism by which DIPs establish persistent infection in cell cultures and re-initiate replication when cells become infected with wt virus. It was found that persistently infected cell cultures, at a later passage, were resistant to homologous virus infection while producing little or no virus. (De and Nayak 1980). However, it is known that the amounts of DIPs remain constant during some of these infections (Moscona 1991). Virulence modulation, the mechanism by which DIPs reduce pathogenesis by mediating humoral immune responses, rather than auto-interference with replication of wt virus (Rabinowitz and Huprikar 1979). 


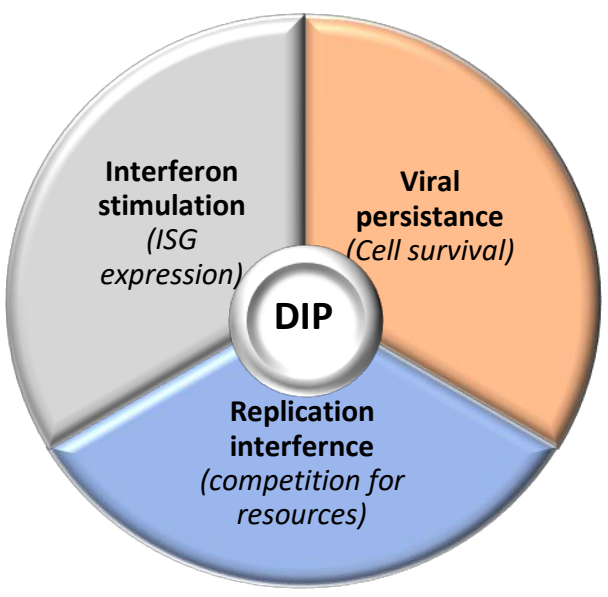

Figure 3.7 Overview of mechanisms that may underly DIP antiviral activity. [inspired by (Vignuzzi and López 2019)]

\subsubsection{Replication Interference}

Replication interference is defined as DIPs outcompeting wt virus for resources required for genome replication (Pathak and Nagy 2009). Replication interference depends on the size of the deletion present in the DI-RNA (Jennings et al. 1983; Nayak et al. 1985; Frensing et al. 2013) since smaller DI-RNAs replicate faster than larger ones and can thus compete with the corresponding wt RNAs with higher efficiency (Nayak et al. 1985; Marriott and Dimmock 2010). DI-RNAs derived from genomic segments 1, 2 and 3 (encoding the polymerase proteins) and harbouring internal deletions are frequently studied (Davis and Nayak 1979; Jennings et al. 1983). The $5^{\prime}$ end of these DI-RNAs must retain at least $150 \mathrm{nt}$ in order to ensure DI-RNA replication (Duhaut and Dimmock 2002). However, DI-RNAs without deletions but harbouring multiple mutations can exist in IAV preparations and may exhibit strong antiviral activity via partially understood mechanisms (Kupke et al. 2019).

The ratio between DIP and wt IAV critically determines replication interference and it has been suggested that based on RNA copy numbers, DIPs have to be administered at a 10,000 to 100,000 -fold excess relative to wt virus in order to exert potent antiviral activity via replication interference (Dimmock and Easton 2014). Finally, replication interference is most potent when the wt viruses are examined from which the DIPs were derived but weak or absent when heterologous viruses are studied (Marriott and Dimmock 2010). More specifically, replication interference of any IAV DI-RNAs probably extends to all wt IAVs. Consequently, DI-244 protects against various strains of IAV subtypes (Dimmock et al. 2008). 
DIPs suppress replication of wt virus (Figure 3.8). Besides genome replication, DIPs are known to exert antiviral activity by affecting packaging steps of IAV. The smaller DI-RNAs are preferentially packaged into new virus particles in comparison to wt RNAs (Liao et al. 2016). DI-244 RNA interferes with replication of several genomic RNAs in cells infected with IAV and mutation of start codons of DI-244 has no effect on interference indicating inhibition is independent of any protein product (Meng et al. 2017). Mutation forms of DIPs, OP7 virus, reduced $\mathrm{HA}$ and infectious titers when co-infected with wt virus by interfering with virus replication (Kupke et al. 2019).

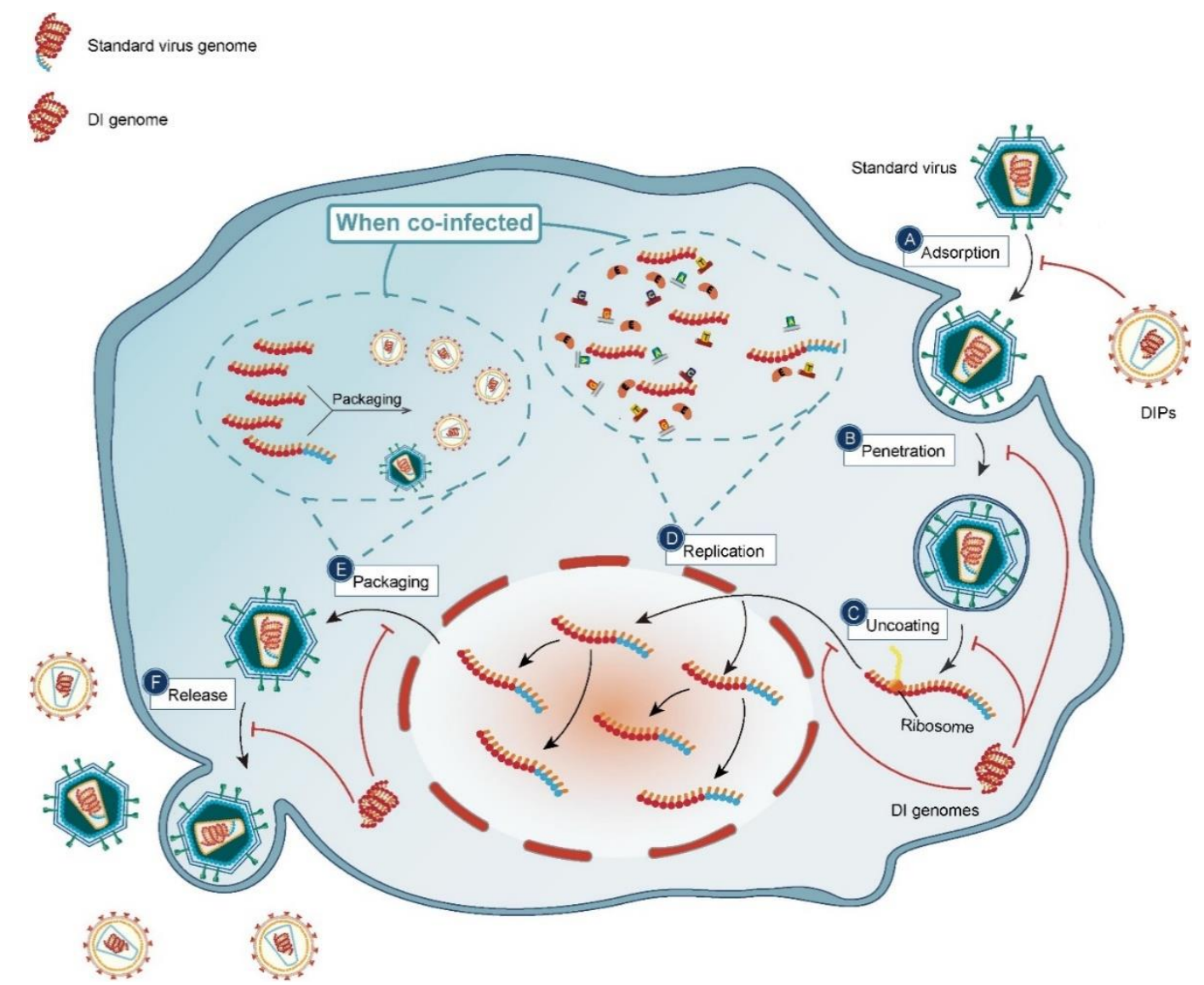

Figure 3.8. DIPs suppress the cycle of wt viruses. The red arrows indicate the inhibitory effect of DIPs. During co-infection with wt virus, DIPs prevent wt virus A-C) from invading cells by binding to cell surface receptors $D$ ) replication cycle by competing for resources E) RNAs from packaging into progeny virions as DI RNAs being shorter are preferentially packaged F) at release step (Yang et al. 2019)

\subsubsection{Interferon induction}

Inhibition of heterologous viruses by DIPs suggested that replication interference is not the sole mechanism underlying DIP antiviral activity and pointed towards activation of innate antiviral defences. Proof of this concept was obtained when type I IFN receptor knock out 
mice were observed to be weakly protected by DI-244 against PVM, although protection against WSN infection was robust (Easton et al. 2011). It was even suggested that DI-244 may protect against all type I IFN-sensitive respiratory viruses in the presence of a functional IFN system. However, induction of IFN by DIPs might not be limited to type I IFN (Easton et al. 2011; Scott et al. 2011a) since type III IFN was shown to be important for protection against IAV and IBV infection in vivo (Mordstein et al. 2010).

\subsubsection{The interferon system}

The innate immune system serves as the first line of defence against pathogens and macrophages, dendritic cells, neutrophils, eosinophils, natural killer (NK) cells are important innate immune cells. The IFN system is a essential component of innate immunity and essential for the defence against viral infections. IFNs were discovered in 1957 by Isaacs \& Lindenmann as a substance produced by cells that interferes with influenza virus infection (Isaacs et al. 1957; Schneider et al. 2014; Isaacs and Lindenmann 1957). IFNs are small proteins secreted by cells (Lengyel 1982) following the recognition of pathogens (Wu and Chen 2014).

\subsubsection{Sensors and signal transducers of the interferon system}

The IFN system recognises pathogen associated molecular patterns (PAMPs) using different pattern recognition receptors (PRRs). PRRs are either endosomal transmembrane proteins or cytosolic proteins and PRRs recognizing nucleic acids are grouped into three types: Toll-like receptor (TLR), cyclic GMP-AMP synthase (cGAS) and RIG-I-like receptors (RLR) (Fensterl et al. 2015). Binding of PAMPs to these PRRs induces conformational changes in the PRRs that result in activation of downstream adaptor molecules.

TLRs are transmembrane proteins containing multiple leucine-rich repeat domains responsible for recognition of PAMPs, such as dsRNA (TLR3) or ssRNA (TLR7, TLR8) (Xagorari and Chlichlia 2008; Gay et al. 2014). They are localized at the plasma membrane or in endosomes and thus mainly responsible for detection of extracellular nucleic acids (Gay et al. 2014). The cytosolic cGAS acts as a DNA detector, which produces cyclic dinucleotides (2'3'-cGAMP) from ATP and GTP upon binding of DNA (Cai et al. 2014).The RLR family consists of three members that include the retinoic acid inducible gene I (RIG-I), melanoma differentiation factor 5 (MDA5), and Laboratory of Genetics and Physiology gene 2 (LGP2) (Baum et al. 2010). RIG-I recognises RNA of various lengths with 5'-triphosphates (5'ppp) at 
the ends (Yoneyama et al. 2015; Weber et al. 2013; Goubau et al. 2014) whereas MDA5 recognises long dsRNAs (Kato et al. 2008). Both RIG-I and MDA5 signal to mitochondrial antiviral signalling protein (MAVS) (Seth et al. 2005). PRR signalling ultimately activates interferon regulatory factor 3 (IRF3) to induce IFN- $\beta$ gene expression (Honda et al. 2006; Paun and Pitha 2007). Subsequently IFN- $\beta$ stimulates a first wave of ISG transcription, including IFN-inducible transcription factor IRF7. Eventually IRF7 activates various IFN- $\alpha$ subtypes, thereby mediating second wave of ISG transcription. (Lazear et al. 2019). In addition to IFN- $\alpha$ and IFN- $\beta$, IRF3 and/or IRF7 induces IFN- $\lambda$ (Osterlund et al. 2007), whereas IRF1 induces IFN- $\lambda 1$ (Odendall et al. 2017)

Indirect evidence from quantification of interferon-inducing and defective particles from DIP preparations indicated that DIPs alone do not induce IFN (Ngunjiri et al. 2012). Another study showed mini viral RNAs (mvRNAs) bind to RIG-I and considerably induce higher IFN expression in comparison to full-length genome or DI-RNA (Te Velthuis et al. 2018). RIG-I plays a crucial role in sensing viral infection and commencing IFN expression (Pichlmair et al. 2006). RIG-I preferentially associates with DI-RNAs in comparison to the corresponding wt RNAs in influenza-infected cells (Baum et al. 2010). RIG-I promotes disassembly of viral polymerase complex by binding to 5'ppp-ds RNA panhandle structure of PB2 exhibiting direct antiviral activity (Weber-Gerlach and Weber 2016).

\subsubsection{Types of interferons and receptors}

Interferons (IFN) are classified into three subtypes: type I (IFN- $\alpha, \beta, \varepsilon, k, \omega, \zeta$ [mice]), type II (IFN- $\gamma$ ) and type III (IFN- $\lambda$ ) based on amino acid sequence and type of receptor they use for signalling. IFN- $\alpha$ and IFN- $\beta$ signal through a heterodimeric IFN-alpha-receptor 1 (IFNAR1) or IFN-alpha-receptor 2 (IFNAR2) complex (Pestka et al. 2004). All tissue cells are capable of producing IFN- $\alpha$ and $\beta$, but a huge amount is produced by plasmacytoid dendritic cells (pDCs) and macrophages during influenza virus infection (Siegal et al. 1999).Type II IFN consists of single molecule, IFN- $\gamma$, produced by immune cells. It signals through homodimeric IFN-gamma-receptor 1 (IFNGR1) and IFN-gamma-receptor 2 (IFNGR2) (Pestka et al. 1997). Type III IFNs, namely IFN- $\lambda 1$, IFN- $\lambda 2$, IFN- $\lambda 3$ are the most recently discovered members of the IFN group (Kotenko et al. 2003; Sheppard et al. 2003). IFN- $\lambda$ is produced by pDCs and monocyte-derived DCs (Coccia et al. 2004). They signal through heterodimeric receptor 
interleukin-10 receptor 2 (IL-10R2) and IFN-lambda-receptor (IFNLR) (Schneider et al. 2014). Type III IFN signalling is restricted to epithelial cells (Sommereyns et al. 2008).

\subsubsection{The JAK/STAT pathway}

In the 1990s a pathway for IFN-induced gene expression was found to be a important one, commonly known as the JAK-STAT signalling pathway (Velazquez et al. 1992; Darnell et al. 1994). Upon binding of IFN to their cell-surface receptors a signalling cascade is triggered leading to drastic changes in the properties of cells, the foremost being induction of an antiviral state (Stark and Darnell 2012).

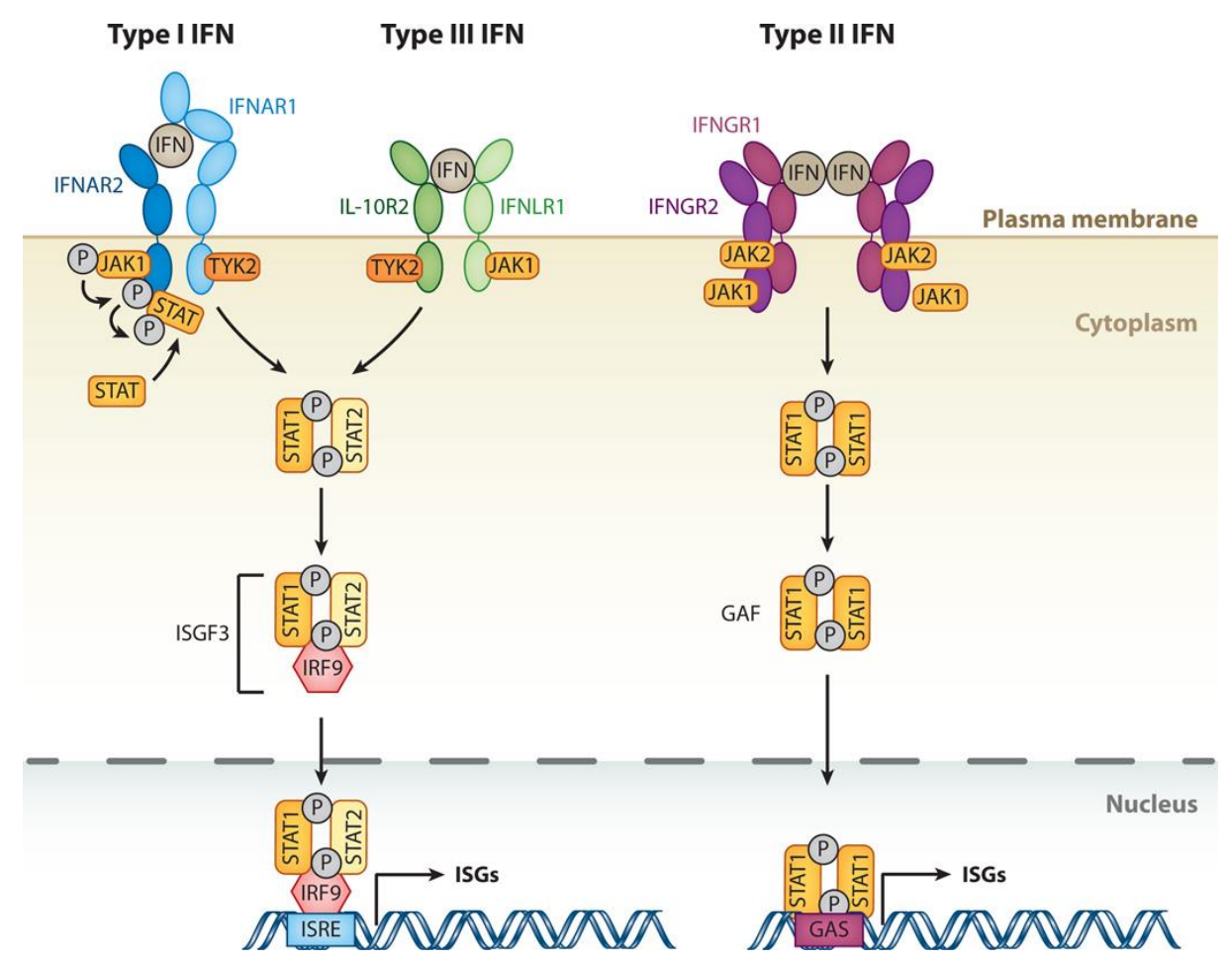

Figure 3.9. The JAK-STAT signalling pathway. Binding of IFN to their respective receptors induces phosphorylation of Janus kinase (JAK) and tyrosine kinase (TYK). This leads to phosphorylation of signal transducer and activator of transcription (STAT). The STATs important for type I and III IFN signalling then bind to the IFN-regulatory factor 9 (IRF9) to form IFN-stimulated gene factor 3 (ISGF3) complex. The STAT1 homodimers crucial for type II IFN signalling form the IFN- $\gamma$ activation factor (GAF) complex. Both complexes translocate to the nucleus to induce antiviral genes (Schneider et al. 2014).

Binding of type I and type III IFNs to their receptors trigger phosphorylation of Janus kinase 1 (JAK1) and tyrosine kinase 2 (TYK2), which in turn phosphorylates the receptors at specific intracellular tyrosine residues. Subsequently, this leads to the phosphorylation of signal transducers and activators of transcription 1 and 2 (STAT 1 and 2). STAT1 and 2 form heterodimers which in turn recruit interferon regulatory factor (IRF9) to form the interferon- 
stimulated gene factor 3 (ISGF3) complex (Schindler et al. 1992; Stark and Darnell 2012). Binding of type II IFN dimers to their receptors lead to phosphorylation of JAK1 and JAK2 tyrosine kinases and transphosphorylation of receptor chains then leads to phosphorylation of STAT1. Phosphorylated homodimers of STAT1 form the IFN-gamma activation factor (GAF). Both ISGF3 and GAF translocate into the nucleus and induce genes regulated by IFNstimulated response elements (ISRE) and gamma-activated sequences (GAS) respectively, resulting in the expression of antiviral genes (Schneider et al. 2014; Wang et al. 2017) (Figure 3.9)

\subsubsection{Signal Transducer and Activator of Transcription (STAT)}

There are seven STAT proteins in mammals STAT 1, STAT2, STAT 3, STAT 4, STAT 5a, STAT5b, and STAT6 (Schindler et al. 1992; Darnell et al. 1994). STATs belongs to a family of transcription factors that are activated in response to cytokines, growth factors and certain peptides (Levy and Darnell 2002). STAT1 is phosphorylated in response to IFN binding to their receptors (Fu et al. 1992) but can also be acetylated by dephosphorylation of tyrosine and methylated by methyl transferase of STAT1 inactivator leading to increased binding affinity (Najjar and Fagard 2010). Studies report that after IFN- $\alpha$ treatment, STAT2 was first recruited via its Src homology region 2 (SH2) domain to phosphotyrosine and then promotes STAT1 phosphorylation (Stark et al. 1998; Park et al. 2000). In the absence of STAT1, STAT2 interacts with IRF9 forming an ISGF3 complex to stimulate ISG expression (Fink and Grandvaux 2013). In STAT1-knockout cells, IFN- $\gamma$ was found to regulate a large panel of genes by mediating via alternative pathways used by their receptors in primary cells (Gil et al. 2001).

\subsubsection{Interferon-stimulated genes}

Interferon-stimulated genes (ISGs) are liable for antiviral, antitumor and immunosuppressive properties of IFN. IFN induces the expression of roughly 400 genes, many of which encode proteins with antiviral activity that are responsible for the IFN-induced antiviral state (Iwasaki and Pillai 2014). ISGs with anti-influenza activity are listed in the Table 3.3

ISGs control viral, bacterial, and parasitic infection by directly targeting pathogens or cellular pathways required for pathogen multiplication (Schneider et al. 2014). Importantly, PRRs, IRFs, JAK2, STAT1/2 and IRF9 are themselves ISGs which further stimulate IFN expression and 
thus augment the IFN response. ISGs with antiviral activity can block early or late steps of the viral replication cycle (Schneider et al. 2014). For example, IFN-induced transmembrane proteins (IFITM) block virus entry (Brass et al. 2009), while viperin inhibits virus budding (Wang et al. 2007).

The murine Myxovirus resistance gene $(M \times 1)$ was the first antiviral gene identified and is considered to be highly important for countering IAV infection. Human cells express $M \times 1$ (also termed $M \times A$ ) and $M \times 2$ (also termed $M \times B$ ) proteins. Both are IFN-induced and belong to the family of GTPases. They block an early post-entry step occurring prior to viral genome replication (Schneider et al. 2014). Mx1 acts against IAV while Mx2 potently inhibits HIV-1 and HIV-2 (Goujon et al. 2013). Mx1 traps the nucleocapsid of IAV and prevents it from reaching the nucleus (Zimmermann et al. 2011). Mx1 consists of a stalk region and a GTPase effector domain which both are essential for self-oligomerization and formation of a ring-like structure. This structure plays an important role in antiviral activity against IAV (Gao et al. 2010; Haller et al. 2010; Haller et al. 2015; Villalón-Letelier et al. 2017). $M x 1$ is transcriptionally upregulated by type I and type III IFN. Infection of STAT1-deficient cells with NS1-deficient IAV did not induce $M x 1$ gene expression, indicating that $M x 1$ expression requires STAT1 signalling and cannot be triggered upon virus infection (Holzinger et al. 2007).

Table 3.3 Anti influenza virus-activity of interferon-stimulated genes [adapted from (Iwasaki and Pillai 2014)]

\begin{tabular}{|c|c|c|}
\hline ISGs & Control mode & Reference \\
\hline OAS & $\begin{array}{l}\text { Inhibits virus by degrading viral RNA and blocking } \\
\text { translation of viral mRNAs } \\
\text { Senses foreign RNA and produces } 2^{\prime}-5^{\prime} \text { adenylic acid } \\
\text { which activates RNaseL that cleaves vRNA }\end{array}$ & (Silverman 2007) \\
\hline PKR & $\begin{array}{l}\text { Blocks translation, activates the NF-KB pathway, } \\
\text { Phosphorylates the } \alpha \text {-subunit of EIF } 2 \alpha \text {, and stabilizes } \\
\text { IFN- } \alpha \text { and IFN } \beta \text { mRNA }\end{array}$ & $\begin{array}{l}\text { (Kumar et al. 1994; } \\
\text { Balachandran et al. } \\
\text { 2000; Sadler and } \\
\text { Williams 2007; Schulz } \\
\text { et al. 2010) }\end{array}$ \\
\hline $\mathrm{CH} 25 \mathrm{H}$ & $\begin{array}{l}\text { Affects virus at host-membrane fusion, protein } \\
\text { maturation of viral structural proteins and of viral } \\
\text { replication enzymes } \\
\text { Blocks viral fusion by converting cholesterol to a soluble } \\
25 \text {-hydroxycholesterol }\end{array}$ & $\begin{array}{l}\text { (Liu et al. 2012; Blanc } \\
\text { et al. 2013) }\end{array}$ \\
\hline IFITM1-3 & Inhibits endocytic fusion events & $\begin{array}{l}\text { (Brass et al. 2009; } \\
\text { Bailey et al. 2012; Jia } \\
\text { et al. 2014) }\end{array}$ \\
\hline
\end{tabular}




\begin{tabular}{|c|c|c|}
\hline ISGs & Control mode & Reference \\
\hline ISG15 & $\begin{array}{l}\text { Targets viral proteins that are newly translated for } \\
\text { ubiquitination }\end{array}$ & $\begin{array}{l}\text { (Lenschow et al. } \\
\text { 2007; Durfee et al. } \\
\text { 2010) }\end{array}$ \\
\hline Viperin & $\begin{array}{l}\text { Forms lipid rafts from which virus buds and prevents } \\
\text { release of influenza virus }\end{array}$ & $\begin{array}{l}\text { (Wang et al. 2007; } \\
\text { Hinson and Cresswell } \\
\text { 2009a, 2009b) }\end{array}$ \\
\hline ISG20 & $\begin{array}{l}\text { Suppresses viral polymerase and exhibits exonuclease } \\
\text { activity essential for anti-IAV activity }\end{array}$ & (Qu et al. 2016) \\
\hline TRIM25 & $\begin{array}{l}\text { Binds vRNPs in the nucleus of infected cells and restrict } \\
\text { the influenza replication. } \\
\text { Inhibits the RNA chain elongation by restricting RNA } \\
\text { movement in polymerase complex }\end{array}$ & $\begin{array}{l}\text { (Meyerson } \\
\text { 2017) }\end{array}$ \\
\hline MOV10 & $\begin{array}{l}\text { Binds to the viral NP to prevent its import into the } \\
\text { nucleus }\end{array}$ & (Zhang et al. 2016) \\
\hline TRIM56 & $\begin{array}{l}\text { Inhibits replication of virus-infected cells in the nucleus } \\
\text { independent of ubiquitin ligase activity where its C- } \\
\text { terminal tail suppresses viral RNA synthesis }\end{array}$ & $\begin{array}{l}\text { (Liu et al. 2016; } \\
\text { Villalón-Letelier et al. } \\
\text { 2017) }\end{array}$ \\
\hline SERPINE1 & $\begin{array}{l}\text { Reduces infectivity of progeny virus by targeting a } \\
\text { plasminogen activator inhibitor } 1 \text { (PA1-1) that inhibits } \\
\text { IAV glycoprotein cleavage }\end{array}$ & (Dittmann et al. 2015) \\
\hline TRIM41 & $\begin{array}{l}\text { Interacts with nucleoprotein of IAV through target } \\
\text { binding site (SPRY domain) to inhibit the infection }\end{array}$ & (Patil et al. 2018) \\
\hline ANP32A & $\begin{array}{l}\text { Overcomes viral polymerase host (avian) restriction by } \\
\text { promoting interaction with the viral polymerase }\end{array}$ & $\begin{array}{l}\text { (Domingues and Hale } \\
\text { 2017) }\end{array}$ \\
\hline CPSF4 & $\begin{array}{l}\text { Affects both viral replication and IAV-associated type I } \\
\text { IFN secretion by interacting with viral NS1 }\end{array}$ & (Dubois et al. 2019) \\
\hline SMARCA2 & $\begin{array}{l}\text { Facilitates antiviral activity of MxA against IAV by being } \\
\text { dependent on ISGs }\end{array}$ & (Dornfeld et al. 2018) \\
\hline TRIM38 & $\begin{array}{l}\text { Regulates negatively TLR3-mediated IFN- } \beta \text { signalling by } \\
\text { targeting TRIF }\end{array}$ & (Xue et al. 2012) \\
\hline CD274 & $\begin{array}{l}\text { Accelerates influenza virus clearance and infection } \\
\text { recovery by blockade in the airways }\end{array}$ & (McNally et al. 2013) \\
\hline TRIM22 & Induces proteasomal degradation of the viral NP & (Di Pietro et al. 2013) \\
\hline
\end{tabular}

OAS, 2'-5'-oligoadenylate synthetase; RNaseL, latent RNase; PKR, protein kinase R; EIF2 $\alpha$, eukaryotic translation initiation factor $2 \alpha$; NF-kB, nuclear factor kappa-light-chain-enhancer of activated B-cells; $\mathrm{CH} 25 \mathrm{H}$, cholesterol 25 hydroxylase; IFN, interferon; IFITM, IFN-induced transmembrane; ISG, interferon stimulated gene; TRIM, tripartite motif-containing protein; MOV10, Moloney leukemia virus 10 homolog; SPRY, SPla and the RYanodine Receptor; ANP32A, acidic nuclear phosphoprotein 32 family member A; CD274, programmed cell death 1 ligand 1 (PDL-1); TLR3, Toll-like receptor 3; TRIF, Toll/interleukin-1 receptor domain-containing adaptor inducing IFN- $\beta$; CPSF4, cellular protein cleavage and polyadenylation specificity factor 4 


\section{Aims}

Seasonal influenza is responsible for half a million deaths every year. Influenza viruses rapidly develop resistance against antivirals and vaccines need to be constantly adapted to the circulating viruses. Therefore, novel antiviral strategies to combat influenza are urgently needed. One novel approach is the use of defective viral particles that harbour deletions in essential genes and interfere with wt virus infection. Such particles are called defective interfering particles (DIPs) and exhibit potential as therapeutic and prophylactic agents. However, so far, production of DIPs was dependent on the use of wt viruses as helper viruses, which raises safety concerns. Moreover, it was incompletely understood how DIPs inhibit influenza virus infection. These questions were to be addressed within the present thesis:

The first aim of this thesis was to develop a system for production of DIPs in the absence of wt virus. Specifically, it was to be investigated whether expressing the viral polymerase protein PB2 in cells allows amplification of DIPs harbouring a deletion in the genomic segment encoding for PB2.

The second aim of this thesis was to obtain insights into how DIPs inhibit influenza virus infection. Most importantly, it was to be determined to what extend interference with viral genome replication and interferon-induction contribute to the antiviral activity of DIPs. 


\section{Manuscripts}

A system for production of defective interfering particles in the absence of infectious influenza A viruses.

(PloS One. March 2019)

Interferon induction and not replication interference is the major determinant of anti-influenza virus activity of defective interfering particles

(Prepared for submission) 


\subsection{First Manuscript}

Bdeir, Najat*; Arora, Prerna*; Gärtner, Sabine; Hoffmann, Markus; Reichl, Udo; Pöhlmann, Stefan*; Winkler, Michael*

A system for production of defective interfering particles in the absence of infectious influenza A viruses.

PloS One. March 2019.

*shared first authorship

Individual contribution: In the following manuscript I conducted experiments for Figure $1 \mathrm{~A}$, B and C; "Stable expression of active PB2 protein in 293T and MDCK cells." for Figure 4; "PB2opt stably expressed in 293T cells is active." for Figure 5 D; "Codon optimization of PB2 results in increased DIP production." and for Figure $6 \mathrm{~A}$; "DI-244 produced in PBopt expressing cell lines exerts anti-IAV activity." 


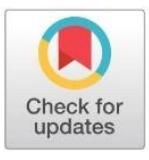

\section{G openaccess}

Citation: Bdeir N, Arora P, Gärtner S, Hoffmann M, Reichl U, Pöhlmann S, et al. (2019) A system for production of defective interfering particles in the absence of infectious influenza A virus. PLOS ONE 14(3): e0212757. https://doi.org/10.1371/journal. pone. 0212757

Editor: Balaji Manicassamy, University of lowa, UNITED STATES

Received: November 22, 2018

Accepted: February 10, 2019

Published: March 1, 2019

Copyright: ๑ 2019 Bdeir et al. This is an open access article distributed under the terms of the Creative Commons Attribution License, which permits unrestricted use, distribution, and reproduction in any medium, provided the original author and source are credited.

Data Availability Statement: All relevant data are within the paper and its Supporting Information file.

Funding: UR and SP were supported by DARPA (defense advanced research projects agency), intercept program.

Competing interests: The authors have declared that no competing interests exist.

\section{RESEARCH ARTICLE}

\section{A system for production of defective interfering particles in the absence of infectious influenza A virus}

\author{
Najat Bdeir ${ }^{1,2 \odot}$, Prerna Arora ${ }^{1,2 \odot}$, Sabine Gärtner ${ }^{1}$, Markus Hoffmann ${ }^{1}$, Udo Reichl $^{3,4}$, \\ Stefan Pöhlmann ${ }^{1,2 \odot *}$, Michael Winkler ${ }^{1 \bullet}$ \\ 1 Infection Biology Unit, German Primate Center-Leibniz Institute for Primate Research, Göttingen, \\ Germany, 2 Faculty of Biology and Psychology, University Göttingen, Göttingen, Germany, 3 Max Planck \\ Institute for Dynamics of Complex Technical Systems, Bioprocess Engineering, Magdeburg, Germany, \\ 4 Otto von Guericke University Magdeburg, Chair for Bioprocess Engineering, Magdeburg, Germany \\ - These authors contributed equally to this work. \\ *spoehlmann@dpz.eu
}

\begin{abstract}
Influenza $A$ virus (IAV) infection poses a serious health threat and novel antiviral strategies are needed. Defective interfering particles (DIPs) can be generated in IAV infected cells due to errors of the viral polymerase and may suppress spread of wild type (wt) virus. The antiviral activity of DIPs is exerted by a DI genomic RNA segment that usually contains a large deletion and suppresses amplification of wt segments, potentially by competing for cellular and viral resources. DI-244 is a naturally occurring prototypic segment 1-derived DI RNA in which most of the PB2 open reading frame has been deleted and which is currently developed for antiviral therapy. At present, coinfection with wt virus is required for production of $\mathrm{DI}-244$ particles which raises concerns regarding biosafety and may complicate interpretation of research results. Here, we show that cocultures of 293T and MDCK cell lines stably expressing codon optimized PB2 allow production of DI-244 particles solely from plasmids and in the absence of helper virus. Moreover, we demonstrate that infectivity of these particles can be quantified using MDCK-PB2 cells. Finally, we report that the DI-244 particles produced in this novel system exert potent antiviral activity against $\mathrm{H} 1 \mathrm{~N} 1$ and $\mathrm{H} 3 \mathrm{~N} 2$ IAV but not against the unrelated vesicular stomatitis virus. This is the first report of DIP production in the absence of infectious IAV and may spur efforts to develop DIPs for antiviral therapy.
\end{abstract}

\section{Introduction}

Influenza A virus infection is responsible for annual influenza epidemics and intermittent pandemics that are associated with significant morbidity and mortality [1]. The ability of IAV to constantly change in response to immune pressure or antiviral treatment limits the effectiveness of currently used antiviral interventions. Thus, vaccines against seasonal influenza need to be annually reformulated and will provide little if any protection against pandemic influenza [1]. Moreover, the effectiveness of antivirals targeting the viral proteins M2 and neuraminidase 
is compromised by the frequent emergence and transmission of resistance mutations [1, 2]. Therefore, novel approaches to combat influenza are urgently needed.

IAVs are enveloped and harbor eight segments of genomic viral RNA. Defective interfering (DI) genomic segments can be generated in IAV infected cells due to errors of the viral polymerase [3, 4]. DI segments usually harbor a large deletion which inactivates the open reading frame encoded by the segment $[3,4]$. The DI segments can interfere with amplification of wild type (wt) segments, potentially by competing for viral and cellular resources required for segment replication. Moreover, DI RNAs can be packaged into progeny virions, termed defective interfering particles (DIPs), and coinfection of target cells with DIPs and IAV will result in preferential amplification of DIPs and suppression of IAV spread [3,4]. This effect has been observed in cell culture [5-8] and in experimentally infected animals [5, 9-15] and may extend to unrelated viruses $[14,16]$, due to the activation of the interferon system $[15,16]$. Moreover, DIP application in a therapeutic or preventive setting prevents or ameliorates influenza in animal models [3-5, 10-16]. In sum, DIPs can be considered natural antivirals produced in the context of infection with IAV and many other viruses and may provide a basis for the development of new strategies for antiviral intervention.

At present, amplification of DIPs requires coinfection of cells with DIPs and wt virus, termed standard or helper virus, which subsequently needs to be inactivated by UV light $[3,4$, $17,18]$. The presence of standard virus poses a safety concern when products for animal and human use are generated and complicates the interpretation of experimental data. Plasmid systems encoding for wt and DI segments along with cell lines expressing the IAV proteins for which the genomic information has been lost upon DI RNA formation might circumvent this issue $[4,19]$. However, expression of the viral polymerase subunit PB2 in trans was found to be insufficient for robust amplification of IAV variants harboring temperature sensitive mutations $[20,21]$ and it has been speculated that similar limitations might apply to the production of DIPs [4]. Moreover, it has been suggested that PB2 expression might be toxic to cells [4]. Therefore, it is currently unknown whether the strategy outlined above might allow for production of segment 1-derived DIPs and at present no system for generation of DIPs in the absence of standard virus has been reported.

DI-244 is a naturally occurring DI-RNA found in hen's eggs [22]. DI-244 is derived from segment 1 , which encodes PB2, and harbors a 1,946 nucleotides comprising deletion [4, 22]. This deletion removes most of the PB2 ORF but leaves the 3' 244 nucleotides and 5' 151 nucleotides of segment 1 intact which are sufficient for segment replication and packaging [4, 22]. Here, we investigated whether coexpression of wt segments 2-8, PB2 protein and DI-244 RNA allows for production of DIPs. Employing a novel DI-244 variant encoding mScarlet-i, we show that DI-244-based DIPs are efficiently produced in cells expressing a codon optimized version of PB2 and that these DIPs exert potent antiviral activity.

\section{Material and methods}

\section{Plasmids and oligonucleotides}

Plasmids for rescue of the A/PR/8/34 (H1N1) strain, pHW191-pHW198, were used throughout this study and have been previously described [23]. To generate a retroviral vector encoding $\mathrm{PB} 2$, the $\mathrm{PB} 2$ open reading frame was amplified from $\mathrm{pHW} 191$ using primers $\mathrm{PB} 2-\mathrm{QCX}$ IP-5N (5- CCGCGGCCGCACCATGGAAAGAATAAAAGAACTAC-3) and PB2-3XBgl (5-GG AGATCTCGAGCTAATTGATGGCCATCCGAAT-3) and cloned into the retroviral vector pQCXIP-mcs using NotI and XhoI [24]. This self-inactivating vector allows constitutive expression of $\mathrm{PB} 2$ and puromycin resistance genes coupled by an internal ribosome entry site (IRES). An optimized sequence of PB2 was generated by hand to maximize sequence deviation 
from PB2 and optimizing codon usage for influenza A virus and humans ( $\mathrm{S} 1$ Fig). This sequence was synthesized and cloned by GeneArt (Regensburg, Germany) and subcloned using NotI and XhoI sites into pQCXIP-mcs. A plasmid for DI-244 rescue was generated by splice overlap PCR, using pHW191 as template and primer pairs fluA AarI-PB2-1G (5- CGA TCACCTGCTCGAGGGAGCGAAAGCAGGTC-3)/IAVseg1-DI244rep-rev (5- AATGAGGAA TCCCCTCAGTTAAGCGGCCGCTGCGGTACCAGATCTCTTCTCCTGTCTTCCTGA-3) and IAVseg1-DI244rep-for (5- TCAGGAAGACAGGAGAAGAGATCTGGTACCGCAGCGGCCGCT TAACTGAGGGGATTCCTCATT-3)/fluA AarI-PB1-2341R (5- CGATCACCTGC TCTCTAT TAGTAGAAACAAGGCATTT-3). The product of the splice overlap PCR was then purified and amplified with the segment specific primer pair fluA AarI-PB2-1G/fluA AarI-PB1-2341R and cloned into pHW2000-GGAarI, using golden gate cloning, generating pHW2000-DI244-mcs [25]. In addition, a construct containing a multiple cloning site (mcs) was generated for later insertion of reporter genes. For this, the PCR fragments were amplified using pHW191 as template and primer pairs fluA AarI-PB2-1G/IAVseg1-DI244rep-rev (5- AATGAGGAATCCCCT CAGTTAAGCGGCCGCTGCGGTACCAGATCTCTTCTCCTGTCTTCC TGA-3) and IAVseg1DI244rep-for (5- TCAGGAAGACAGGAGAAGAGATCTGGTACCGCA GCGGCCGCTTAACTG AGGGGATTCCTCATT-3)/ fluA AarI-PB1-2341R followed by splice overlap joining and golden gate cloning. As reporter gene, $\mathrm{mScarlet}-\mathrm{i}$ without internal SalI and NotI sites and fused to the porcine teschovirus-1 (PTV1) 2A sequence (GATNFS LLKQAGDVEENPGP) was cloned into the mcs as a BgIII/NotI fragment. In this way, a PB2 (aa 1-41)-2A-mScarlet-i ORF was generated, which allows the detection of the presence of DI-244 via mScarlet-i fluorescence. The template for $\mathrm{mScarlet}-\mathrm{i}$, pmScarlet-i_C1, was a gift from Dorus Gadella (Addgene plasmid \# 85044) [26]. The integrity of PCR-amplified, cloned sequences was verified by sequence analysis.

\section{Cells and viruses}

All cells were cultured at $37^{\circ} \mathrm{C}$ and $5 \% \mathrm{CO}_{2} .293 \mathrm{~T}$ human embryonic kidney cells and Vero cells were maintained in Dulbecco's Modified Eagle Medium (DMEM; Gibco) containing 10\% fetal bovine serum (FBS, Gibco), penicillin (Pen, $100 \mathrm{IU} / \mathrm{ml}$ ) and streptomycin (Strep, $100 \mu \mathrm{g}$ / $\mathrm{ml})$. 293T cell lines stably expressing PB2 were grown in the presence of $1 \mu \mathrm{g} / \mathrm{ml}$ puromycin. Madin-Darby canine kidney cells (MDCK) were cultured in Glasgow's MEM (GMEM) with $10 \%$ fetal bovine serum (FBS, Gibco) and Pen/Strep. All cell lines were obtained from collaborators and were regularly checked for mycoplasma contamination. MDCK cells stably expressing PB2 or PB2opt were cultivated in the presence of $1.5 \mu \mathrm{g} / \mathrm{ml}$ puromycin. Influenza A viruses A/Panama/2007/99 (H3N2) [24] and A/PR/8/34 (H1N1) produced in embryonated chicken eggs were used to assess the antiviral activity of DIPs. We further employed a recombinant vesicular stomatitis virus (VSV) that expresses a dual reporter consisting of eGFP and firefly luciferase from an additional transcription unit located between the open reading frames for the viral glycoprotein and polymerase [27].

\section{Production of retroviral vectors}

The production of MLV particles for transduction of cells followed an established protocol $[25,28]$. Briefly, 293T cells seeded in T25 flasks were transfected with $6 \mu \mathrm{g}$ of retroviral vector (e.g. pQCXIP-PB2), $3 \mu \mathrm{g}$ MLV-gag-pol plasmid and $3 \mu \mathrm{g}$ VSV-G expression plasmid, employing the calcium phosphate transfection method. The culture medium was exchanged at $8 \mathrm{~h}$ after transfection. After $48 \mathrm{~h}, \mathrm{MLV}$ particle-containing supernatant was harvested, cleared by passing through a $0.45 \mu \mathrm{m}$ filter, aliquoted and then stored at $-80^{\circ} \mathrm{C}$. 


\section{Transduction and selection of cell lines}

For retroviral transduction, cells were seeded in 96-well plates at 5,000 (MDCK) or 10,000 (293T) cells/well in $50 \mu \mathrm{l}$ cell culture medium. On the next day, $50 \mu \mathrm{l}$ of supernatant containing MLV particles was added per well followed by spinoculation at 4,000 $\times \mathrm{g}$ for $30 \mathrm{~min}$ for enhancement of transduction [29]. Two days after transduction, the cells were detached and transferred into 24-well plates containing cell culture medium supplemented with $1 \mu \mathrm{g} / \mathrm{ml}$ (293T) and $1.5 \mu \mathrm{g} / \mathrm{ml}$ (MDCK) puromycin. In parallel, non-transduced cells were treated similarly to control for effective cell killing by the antibiotics.

\section{Mini-replicon assay}

$293 \mathrm{~T}$ were seeded at a cell density of $2 \times 10^{5}$ cells/well in 12 -well plates. The following day, the cells were transfected using the calcium phosphate method. The concentrations of plasmids to be transfected were largely adapted from published work [30]: $10 \mathrm{ng}$ of pCAGGS plasmids encoding viral RNA polymerase proteins (PB2, PB, PA) and $100 \mathrm{ng}$ of plasmid encoding NP were cotransfected with $50 \mathrm{ng}$ of plasmid pPolI-Luc, which encodes the firefly luciferase reporter gene flanked by the noncoding regions of segment 8 of A/WSN/33. Empty plasmid was used to ensure that all transfections were conducted with the same total amount of plasmid DNA. For analysis of functionality of PB2 in 293T cells stably expressing this protein, transfection was carried out as described above but the plasmid encoding PB2 was omitted. As control, the plasmid encoding PB1 was omitted. The cells were washed at 6-8 h after transfection and harvested at $24 \mathrm{~h}$ post transfection. Luciferase activities in cell lysates were measured using the Plate Chameleon V plate reader (Hidex) and Microwin 2000 software.

\section{Immunoblot}

For analysis of PB2 expression in 293T and MDCK cells, the cells were seeded in 6-well plates, incubated for $24 \mathrm{~h}$, harvested and lysed in $200 \mu \mathrm{L}$ of Laemmli SDS-PAGE sample buffer (5\% glycerine, $1 \%$ SDS, $2.5 \%$ B-mercaptoethanol, 0.5\% Bromophenol blue, 0.5 mM EDTA,0.5M Tris pH 6.8). Samples were heated to $95^{\circ} \mathrm{C}$ for $10 \mathrm{~min}$ and separated via SDS-PAGE using $12.5 \%$ polyacrylamide gels. Proteins were then transferred onto a nitrocellulose membrane (GE health care) using a Mini-PROTEAN Tetra Cell (BioRad) powered at $110 \mathrm{~V}$ for 90 minutes. Membranes were blocked with $5 \%$ skimmed milk diluted in PBS-Tween and incubated with primary rabbit polyclonal antibodies against PB2 (1:1,000, GenTex, Irvine, USA) overnight at $4^{\circ} \mathrm{C}$. Subsequently, membranes were washed and incubated with anti-rabbit HRP (horseradish peroxidase)-conjugated secondary antibodies (1:10,000, Dianova) for one hour. Finally, chemiluminescent substrate HRP juice plus (P.J.K.) was added onto the membrane and bands were visualized using a ChemoCam imager (INTAS). In order to detect $B$-actin, the membrane was subsequently stripped using stripping buffer (62.5 mM Tris $\mathrm{HCl} \mathrm{pH} \mathrm{6.8,2 \%} \mathrm{SDS,} 100 \mathrm{mM}$ ß-mercaptoethanol) for $30 \mathrm{~min}$ at $5^{\circ} \mathrm{C}$, washed three times with PBS-Tween, and incubated with anti $B$-actin mouse (1:500 Sigma-Aldrich) overnight. The membrane was then washed and incubated with antimouse HRP-conjugated secondary antibody (1:10,000, Dianova) for one hour. HRP juice plus was added and bands were visualized as previously described. Quantification of PB2 and PB2opt expression was carried out using the program ImageJ (FIJI distribution) [31]. In order to normalize data, signals measured for PB2/PB2opt were divided by those measured for beta-actin.

\section{Production of defective interfering particles}

For DIP production, a coculture of 200,000 MDCK cells and 700,000 293T cells stably expressing PB2 was seeded in T25 flasks. The next day, cells were cotransfected via the calcium 
phosphate method with $1 \mu \mathrm{g}$ each of plasmids encoding DI-244-mScarlet-i and wt IAV genomic segments 2-8. Culture medium was changed at $8 \mathrm{~h}$ post transfection. At $48 \mathrm{~h}$ post transfection, cells were washed with phosphate buffered saline (PBS) without calcium and magnesium and DMEM medium supplemented with $0.2 \%$ BSA (MACS BSA), $0.5 \mu \mathrm{g} / \mathrm{ml}$ tosyl-phenylalanychloromethyl-ketone (TPCK)-trypsin (Sigma), penicillin (100 IU/ml) and streptomycin (100 $\mu \mathrm{g} /$ $\mathrm{ml}$ ) was added. As negative control, transfection of parental MDCK and 293T cells was analyzed. Supernatants were harvested from all cultures at 4, 6, 8 and 10 days post transfection, cleared by centrifugation at $4,000 \mathrm{rpm}$ for $10 \mathrm{~min}$ to remove debris, aliquoted and stored at $-80^{\circ} \mathrm{C}$. Infectivity of supernatants was analyzed by focus formation assay as described $[25,32]$ but using MDCK cells expressing PB2 or PB2opt as targets. In brief, MDCK-PB2/PB2opt cells seeded in 96-well plates were washed and incubated for $1 \mathrm{~h}$ with serial dilutions of DIP-containing supernatants. Thereafter, supernatants were removed and infection medium (GMEM with $0.2 \%$ BSA and Pen/ Strep) supplemented with $0.5 \%$ methylcellulose and $0.5 \mu \mathrm{g} / \mathrm{ml} \mathrm{TPCK-trypsin} \mathrm{was} \mathrm{added.} \mathrm{Plates}$ were incubated for $72 \mathrm{~h}$ and then stained using anti IAV polyclonal antibody (Millipore).

\section{Immunofluorescence}

Images were taken on a Zeiss LSM800 equipped with a 10x/0.45 plan-apochromat objective, $488 \mathrm{~nm}$ and $561 \mathrm{~nm}$ diode lasers and ZEN imaging software (Zeiss). Fluorescent signals (red channel, $561 \mathrm{~nm}$ laser) were detected with GaAsP detector employing the same sensitivity for all images of a series, while bright field signals were recorded with an ESID detector (photodiode) with individually adjusted sensitivity.

\section{Analysis of antiviral activity of DIPs}

To test antiviral activity of DIPs against IAV and unrelated VSV, we performed infection experiments in the presence of DIP-containing or DIP-free supernatants and subsequently compared viral titers in the culture supernatants. For this, MDCK cells were seeded in 96-well plates at a density of 10,000 cells/well. On the next day, DIP-containing supernatants or DIP-free control supernatants were 10 -fold serially diluted. Subsequently, MDCK cells were washed twice with PBS and $50 \mu \mathrm{l}$ of the respective supernatants were mixed with $50 \mu \mathrm{l}$ of virus and the mixture inoculated onto the MDCK cells. After a $1 \mathrm{~h}$ incubation, $100 \mu \mathrm{l}$ of fresh infection medium supplemented with $0.5 \mu \mathrm{g} / \mathrm{ml}$ TPCK-trypsin was added and the cells were further incubated for $24 \mathrm{~h}$ (VSV) or $72 \mathrm{~h}$ (IAV) before viral titers in the culture supernatants were determined. Virus titration was performed on confluent monolayers of MDCK (IAV) or Vero (VSV) cells that were grown in 96-well plates. After aspiration of the culture medium, cells were washed twice with PBS and inoculated with $50 \mu \mathrm{l}$ of 10-fold serial dilutions of the culture supernatants of IAV or VSV infected MDCK cells. After $1 \mathrm{~h}$ of incubation with IAV containing supernatants, the medium was removed and $100 \mu \mathrm{l}$ infection medium supplemented with $1 \%$ Avicel and $0.5 \mu \mathrm{g} / \mathrm{ml}$ TPCK-trypsin (IAV/MDCK) was added per well. After $1 \mathrm{~h}$ incubation with VSV-containing supernatants, $200 \mu \mathrm{l}$ infection medium supplemented with $0.5 \%$ methylcellulose (VSV/Vero) were added on top, and the cells were further incubated for $24 \mathrm{~h}$. IAV titers were quantified by antibody staining, using the focus formation assay as previously described $[25,32]$. In order to quantify VSV titers, eGFP-positive foci were counted under the fluorescence microscope. All titers are given as focus forming units per $\mathrm{ml}$ (ffu/ml).

\section{Results}

\section{Generation of 293T and MDCK cells stably expressing functional PB2}

We sought to determine whether DI-244 particles can be amplified in the absence of standard virus if producer cells are engineered to express PB2. For this, we first used retroviral 
transduction and selection antibiotics to generate 293T and MDCK cell lines stably expressing PB2. Immunoblot revealed that the cell lines obtained by selection expressed robust levels of PB2 (Fig 1A and Fig 1B). In order to analyze whether PB2 is functional in these cells, we employed a mini-replicon system, which measures the amplification of a firefly luciferase encoding IAV reporter segment upon coexpression of PB2, PB1, PA and NP [30]. We found that transfection of 293T-PB2 cells with a plasmid encoding the reporter segment alone yielded luciferase activity in the background range while cotransfection of PB2, PB1, PA and NP expression plasmids increased luciferase activity more than 1,000-fold (Fig 1C). Importantly, this increase was not observed when the PB1 plasmid was omitted while omission of the PB2 plasmid had no impact on reporter activity (Fig 1C). Thus, the PB2 protein stably expressed in 293T cells was functional. Unfortunately, similar studies in MDCK cells were not feasible due to the low transfectability of these cells.

\section{PB2 expression allows production of infectious DI-244 in the absence of standard virus}

We next investigated whether the 293T-PB2 and MDCK-PB2 cells allowed the generation of DI-244 particles, using the experimental setup depicted in Fig 2A. In order to be able to visually inspect DI-244 production and spread, we generated a DI-244 variant that encodes for $\mathrm{mScar}-$ let-i, a red fluorescent protein [26]. Transfection of a mixture of 293T/MDCK cells with plasmids encoding IAV wt segments 2-8 jointly with a plasmid encoding DI-244-mScarlet-i resulted in occasional and moderate red fluorescence (Fig $3 \mathrm{~A}$ ). In contrast, frequent and prominent red fluorescence was observed in 293T-PB2/MDCK-PB2 cocultures (Fig 3A), indicating that the stably expressed PB2 promoted amplification of the DI-244-mScarlet-i DI RNA.

In order to examine whether amplification of the DI-244-mScarlet-i DI RNA resulted in the production of infectious DIPs, the supernatants of the transfected 293T-PB2/MDCK-PB2 cells were inoculated onto MDCK-PB2 cells (Fig 2B). As controls, the supernatants were also added to MDCK wt cells. Inoculation of MDCK-PB2 cells with supernatants from 293T-PB2/ MDCK-PB2 cells resulted in infection of the target cells, as determined by expression of $\mathrm{mScarlet}-\mathrm{i}$ (Fig $3 \mathrm{~B}$ ). The number of $\mathrm{mScarlet}-\mathrm{i}$-positive cells was concentration dependent and supernatants taken at 6 days post transfection from DIP producing cells contained the highest amount of infectivity (Fig 3B). Finally, no cells with prominent red fluorescence were detected under control conditions, indicating that DIPs were only infectious for MDCK-PB2 but not MDCK wt cells.

We next asked whether DI-244 production could be quantified by focus formation assay, which is based on detection of IAV antigens by antibody staining and is frequently employed to measure IAV infectivity. Moreover, we examined whether results obtained in the focus formation assay would match those obtained upon counting of foci based upon red fluorescence. Foci were observed in MDCK-PB2 but not in MDCK control cells, confirming that DIP infectivity requires PB2 expression in target cells. Quantification of DIP infectivity by focus formation assay revealed that maximum titers of roughly $1 \times 10^{3}$ DIPs per ml were obtained and counting red fluorescent foci yielded roughly comparable results (Fig $3 \mathrm{~B}$ and Fig 3C). Thus, expression of $\mathrm{PB} 2$ is sufficient for DI-244 production in the absence of helper virus but production efficiency is moderate.

\section{Codon optimization of PB2 allows increased PB2 expression and DIP production}

DIP titers of $1 \times 10^{3}$ particles per $\mathrm{ml}$ are low and may limit experimentation. Therefore, we next asked whether alteration of codon usage for PB2 expression might increase PB2 
A)

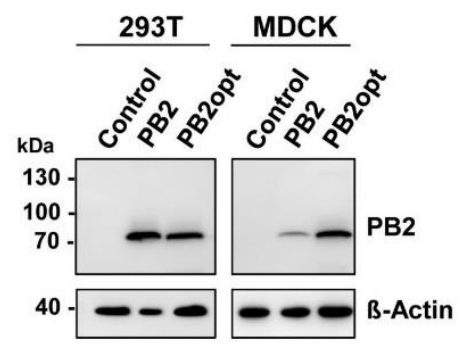

B)

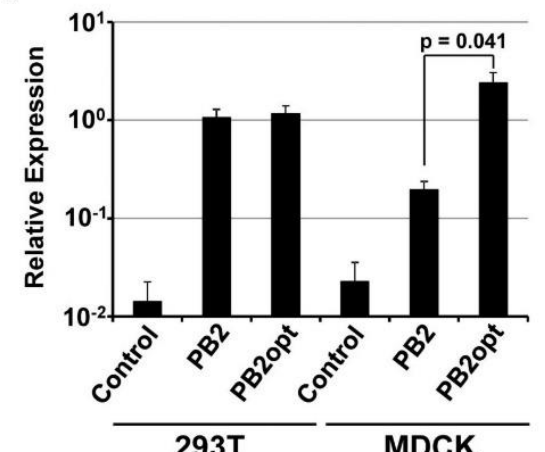

C)

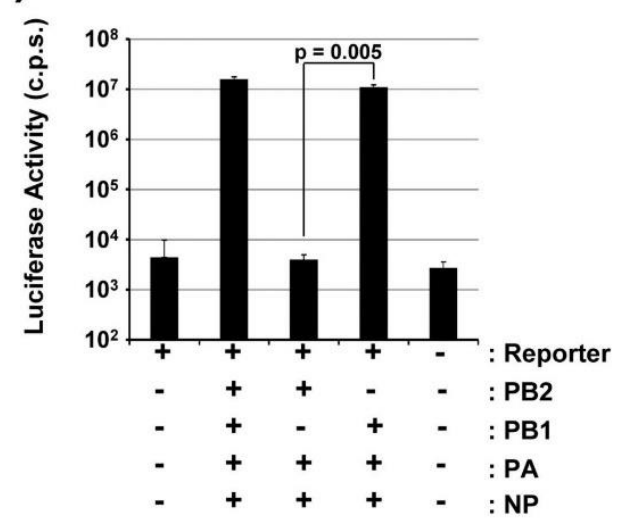

Fig 1. Stable expression of active PB2 protein in 293T and MDCK cells. (A) 293T and MDCK cells engineered to stably express PB2 or codonoptimized PB2 (PB2opt) were analyzed for PB2 expression by immunoblot using anti-PB2 antibody. Detection of beta-actin expression served as loading control. Similar results were obtained in four separate experiments. (B) The average of five experiments conducted as described for panel A and quantified via the ImageJ program is shown. Signals measured for PB2 or PB2opt were normalized against those measured for beta-actin. Error bars indicate standard error of the mean (SEM). Two tailed paired students t-test was used to assess statistical significance. (C) $293 \mathrm{~T}$ cells stably expressing PB2 were cotransfected with plasmids encoding an IAV luciferase reporter segment and the indicated IAV proteins. Luciferase activities in cell lysates were determined at $24 \mathrm{~h}$ post transfection. The results of a representative experiment carried out with triplicate samples are shown. Error bars indicate standard deviation. Two tailed paired students $t$-test was used to assess statistical significance. Similar results were obtained in three separate experiments. C.p.s., counts per second.

https://doi.org/10.1371/journal.pone.0212757.g001

expression efficiency and DIP production. For this, we modified the codons in the PB2 expression plasmid (S1 Fig) to reflect codon preferences of human genes and IAV. As a second criterion for codon choice, we opted for maximal sequence difference between the A/PR/8/ 34-based sequence previously used for PB2 expression and the newly generated, optimized PB2 sequence (PB2opt), in order to prevent potential recombination events. 293T and MDCK cells were engineered to stably express PB2opt and immunoblot revealed that expression levels of PB2opt in MDCK but not 293T cells were higher than those obtained upon expression of non-codon-optimized PB2 (Fig 1A and Fig 1B). Moreover, growth of PB2opt cells was comparable to that of control cells and $\mathrm{PB} 2$ opt expression was readily detectable after multiple passages, suggesting that expression was not associated with overt cytotoxicity. Finally, analysis of 


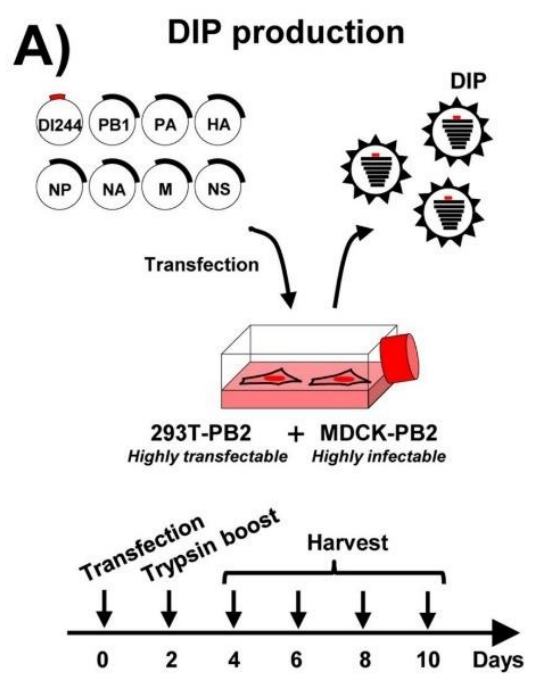

B) DIP quantification
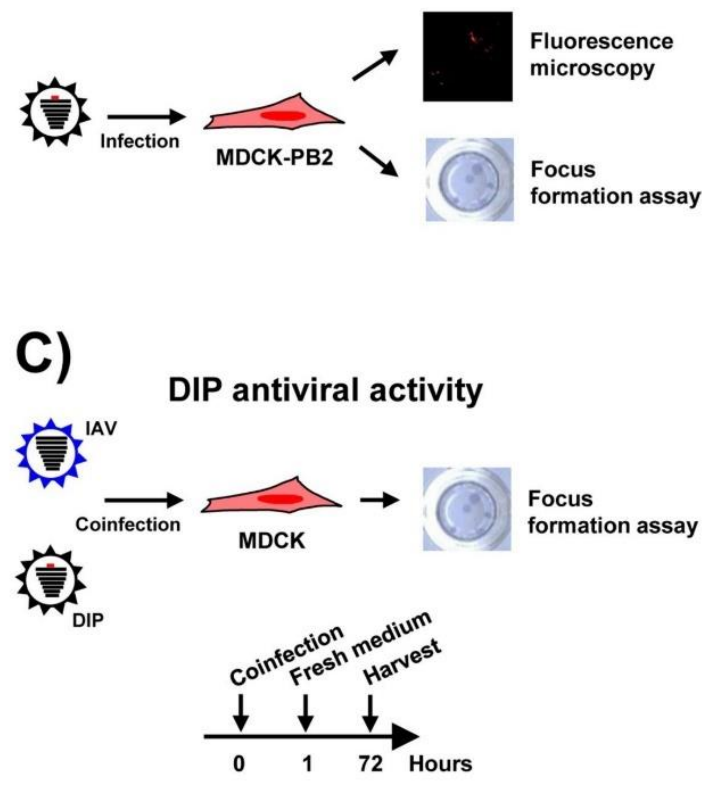
293T-PB2opt cells in the mini-replicon assay showed that PB2opt supported IAV segment replication (Fig 4).

Next, we examined whether PB2opt supports DIP production with higher efficiency than unmodified PB2. Efficient DI-244-mScarlet-i DI RNA amplification was observed in transfected PB2opt cells (not shown) and supernatants obtained from these cells were highly infectious for MDCK-PB2opt cells even when diluted 1:1,000 (Fig 5A). In contrast, the supernatants were not infectious for MDCK cells (Fig 5A). Moreover, a direct comparison of 293T-PB2/MDCK-PB2 and 293T-PB2opt/MDCK-PB2opt cells for production of infectious DIPs and for DIP amplification upon infection revealed that the PB2opt cells were more efficient. Thus, more red fluorescent cells were observed when supernatants from PB2 expressing cells were added to MDCK-PB2opt as compared to MDCK-PB2 cells (Fig 5B). Similarly, supernatants from PB2opt cells were more infectious for target MDCK-PB2opt cells as compared to MDCK-PB2 cells. In keeping with this observation, quantification of production of infectious DIPs by focus formation assay and counting of red fluorescent cells revealed that at least $80 \%$ of foci (identified by antibody staining) were positive for mScarlet-i, as expected, and that PB2opt cells produced up to $4 \times 10^{6}$ infectious DIPs per $\mathrm{ml}$ and thereby exceeded titers obtained with PB2 cells $\left(2,5 \times 10^{3}\right)$ by $\sim 1,500$-fold (Fig $5 \mathrm{C}$ and Fig 5D).

\section{DI-244 produced in the absence of standard virus exerts antiviral activity}

DI-244 can inhibit spread of diverse IAVs and, likely via induction of interferon (IFN), may also inhibit spread of unrelated viruses [3,4]. In order to investigate the antiviral activity of DI-244-mScarlet-i, we first analyzed whether DI-244-mScarlet-i produced in PB2opt cells interfered with the spread of a homologous IAV, A/PR/8/34, in MDCK cells (Fig 2C). For this, MDCK cells were coinfected with the indicated dilutions of DI-244 containing supernatants and A/PR/8/34 at an MOI of 0.1,0.01 and 0.001 (Fig 6A). This resulted in IAV/DIP ratios of approximately 1:10 (undiluted DIP containing supernatants, IAV at MOI 0.1), 1:100 (undiluted DIP containing supernatants, IAV at MOI 0.01) and 1:1,000 (undiluted DIP containing supernatants, IAV at MOI 0.001), respectively. The supernatants from 293T/MDCK wt cells transfected with plasmids for DI-244 production were used as negative control. The control supernatants did not appreciably interfere with $\mathrm{A} / \mathrm{PR} / 8 / 34$ infection while supernatants from PB2opt cells efficiently blocked IAV infection in a concentration dependent manner, with highest antiviral activity observed at an IAV/DIP ratio of 1:1,000 (Fig 6A). Specifically, infection efficiency relative to untreated virus (set as $100 \%$ ) was $1 \pm 0.5 \%$ in the presence of DIP containing supernatants at a dilution of $10^{\circ}$ and $93 \pm 13 \%$ in the presence of control supernatants (average of six independent experiments). Moreover, DI-244 containing supernatants also inhibited infection by A/Panama/2007/99 (H3N2) in a concentration dependent manner (Fig 6B), in keeping with the concept that DI-244 exerts broad anti-IAV activity [3, 4]. Finally, DI-244 containing supernatants did not inhibit VSV infection (Fig 6C), indicating that DI-244 neither interfered with VSV genome replication nor altered viral control by a potential IFN response in MDCK cells. These results show that DI-244 produced in PB2opt expressing cells exerts potent anti-IAV activity. 
A)

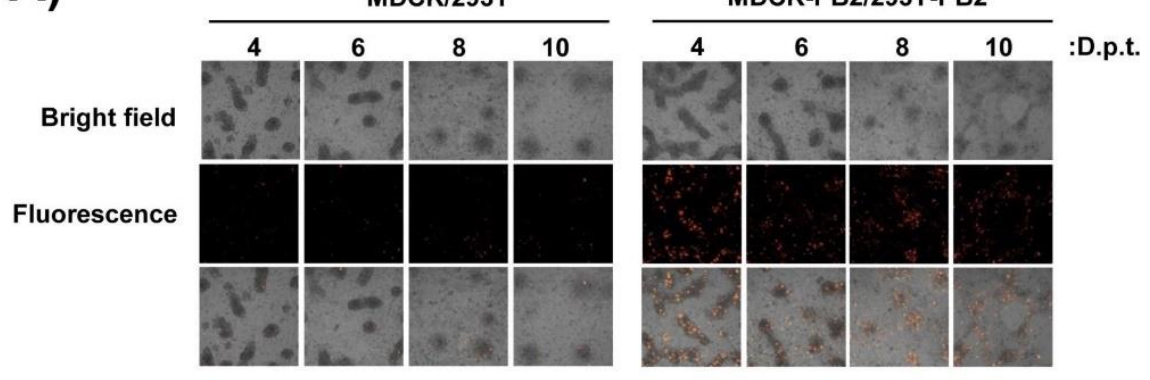

B)

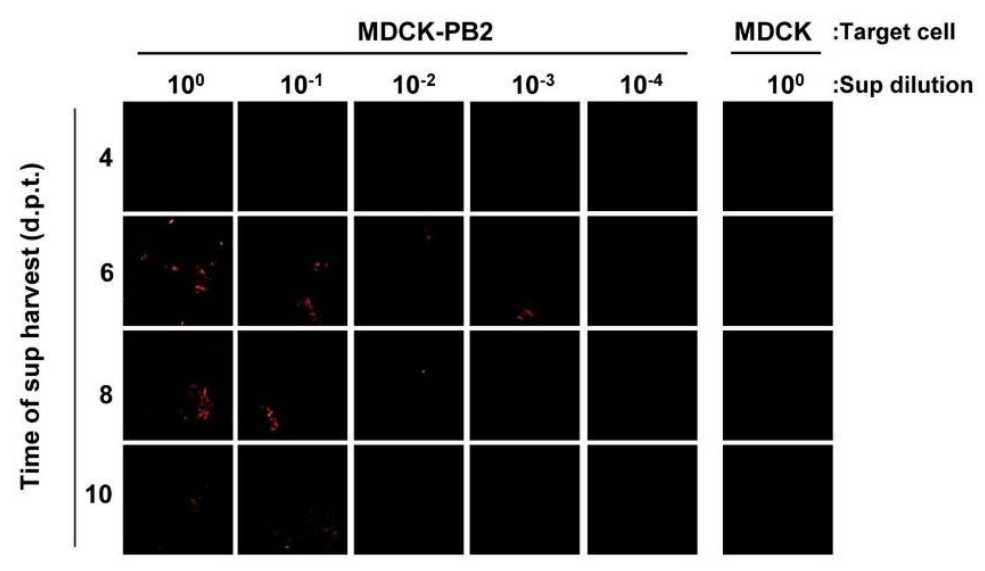

C)

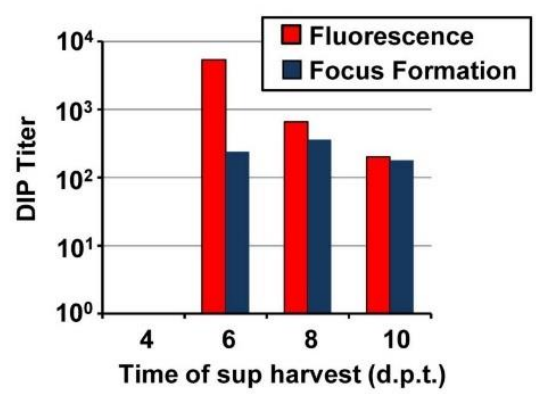

Fig 3. Production of DI-244 particles in PB2 expressing cell lines. (A) Cocultures of 293T-PB2/MDCK-PB2 cells were transfected with plasmids encoding wt IAV segments 2-8 and DI-244-mScarlet-i. The presence of red fluorescence at 4, 6, 8 and 10 days post transfection was analyzed using confocal microscopy. (B) MDCK control and MDCK-PB2 cells were infected with serially diluted DI-244 containing supernatants harvested at the indicated time points and produced as described in panel A followed by removal of supernatants and addition of methyl cellulose overlay. Thereafter, the presence of red fluorescent cells was analyzed at $72 \mathrm{~h}$ post infection using confocal 
microscopy. (C) The number of infected cells (as determined by red fluorescence) in panel B was quantified. In parallel, infection of cells was analyzed by focus formation assay and the number of foci quantified. The results of a representative experiment are shown in panels A-C and were confirmed in two separate experiments.

https://doi.org/10.1371/journal.pone.0212757.g003

\section{Discussion}

The generation of DIPs in IAV infected cells has been recognized by von Magnus several decades ago [33] and DIPs hold promise as novel antiviral agents [3, 4]. However, exploitation of DIPs for antiviral therapy requires efficient production systems that do not depend on the presence of standard virus. Here, we report a DI-244 variant encoding a fluorescent protein that permits monitoring of DIP production. Moreover, we demonstrate that cells expressing

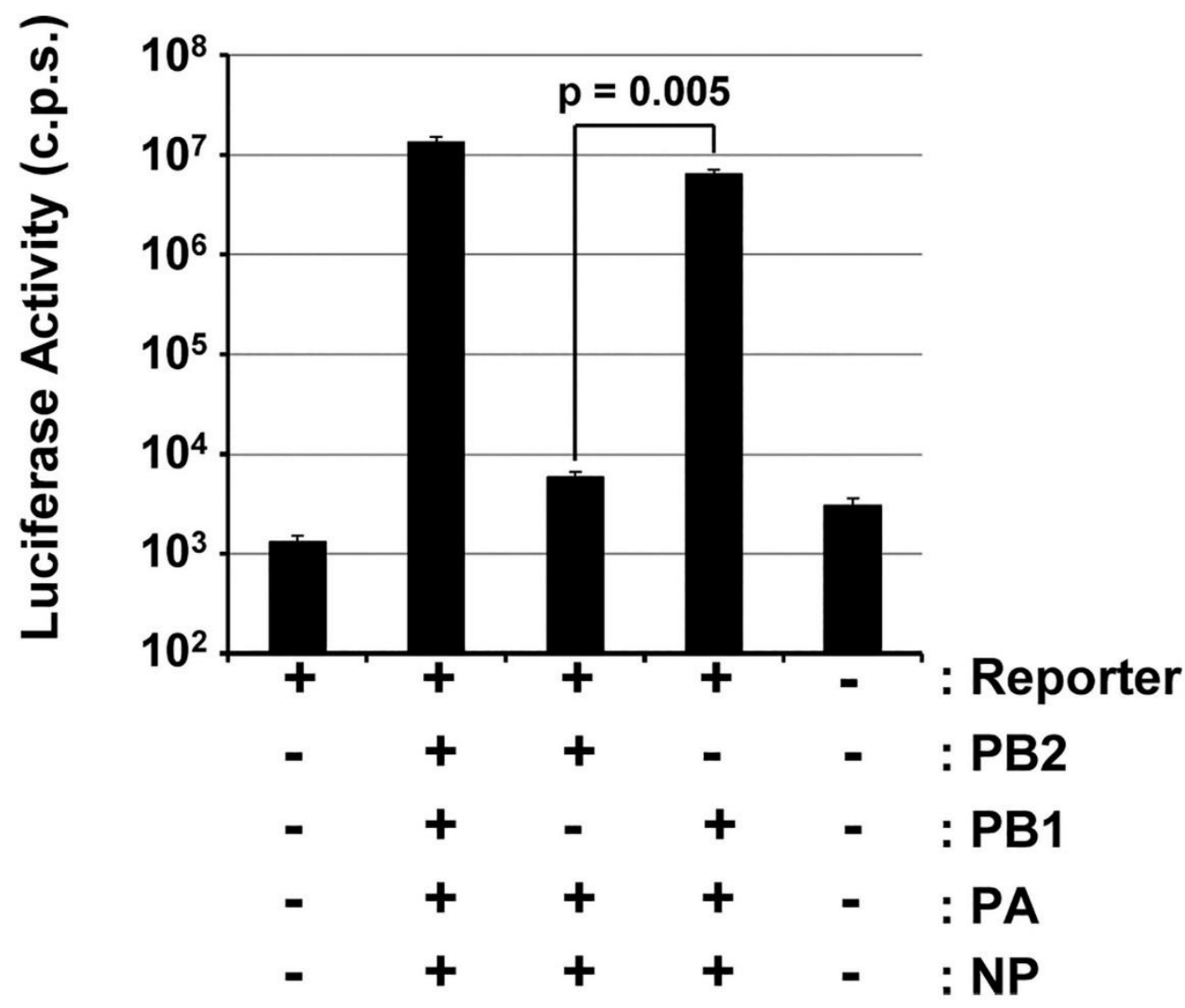

Fig 4. PB2opt stably expressed in 293T cells is active. 293T cells stably expressing PB2opt were cotransfected with plasmids encoding an IAV luciferase reporter segment and the indicated IAV proteins. Luciferase activities in cell lysates were determined at $24 \mathrm{~h}$ post transfection. The results of a representative experiment carried out with triplicate samples are shown. Error bars indicate standard deviation. Similar results were obtained in three separate experiments. Two tailed paired students t-test was used to assess statistical significance. C.p.s., counts per second.

https://doi.org/10.1371/journal. pone.0212757.g004 
A)

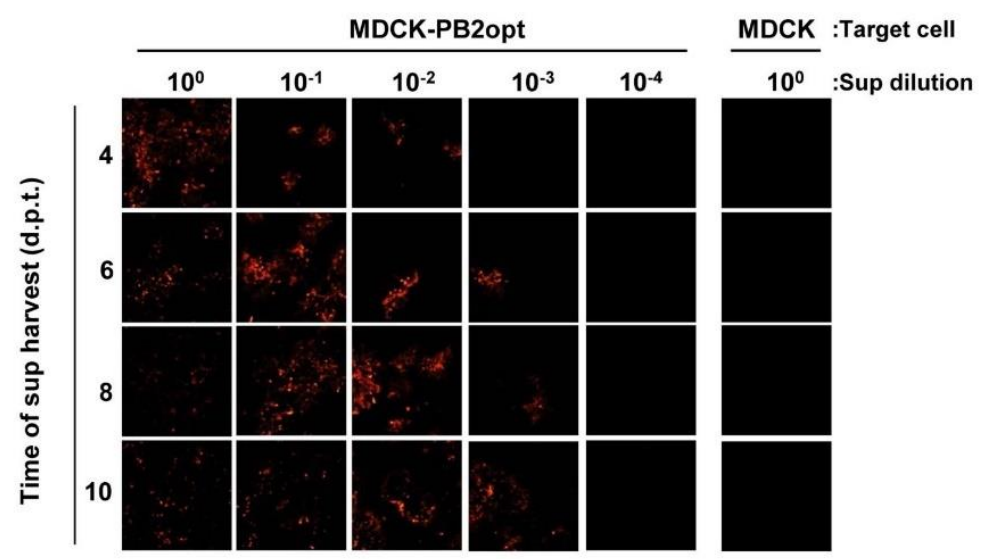

B)

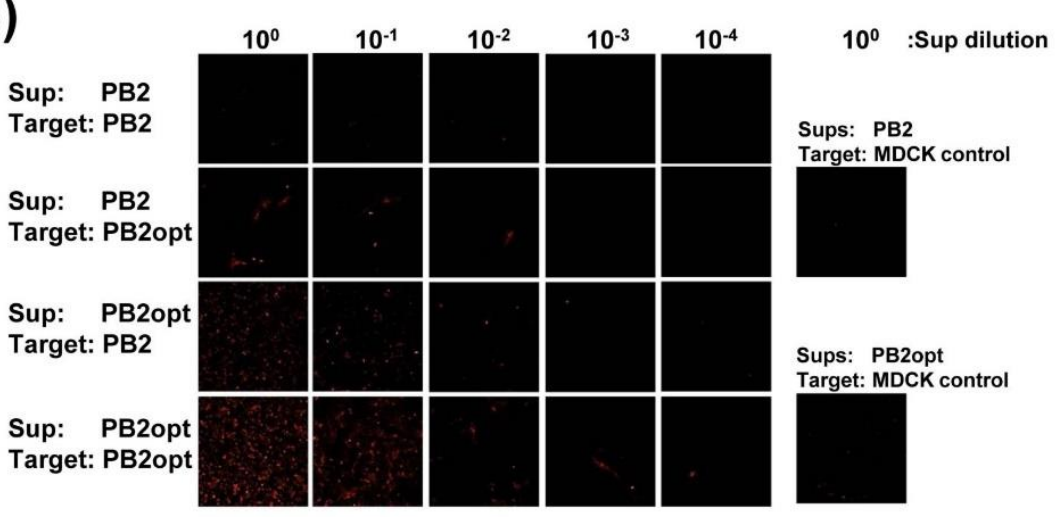

C)

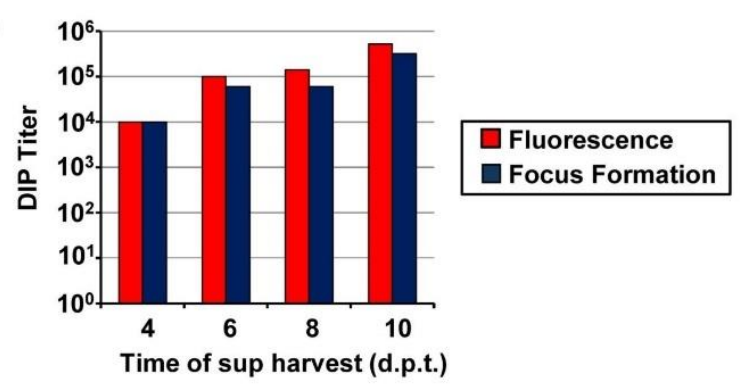

D)

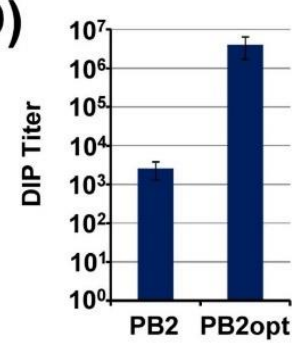


Fig 5. Codon optimization of PB2 results in increased DIP production. (A) DI-244 containing supernatants were produced in Fig 5. Codon optimization of PB2 results in increased DIP production. (A) DI-244 containing supernatants were produced in
293T-PB2opt/MDCK-PB2opt cells and harvested at the indicated time points as described for panel A of Fig 2. Subsequently, MDCK 293T-PB2opt/MDCK-PB2opt cells and harvested at the indicated time points as described for panel A of Fig 2. Subsequently, MDCK
control and MDCK-PB2opt cells were infected with serially diluted DI-244 containing supernatants followed by removal of supernatants control and MDCK-PB2opt cells were infected with serially diluted DI-244 containing supernatants followed by removal of supernatants
and addition of methyl cellulose overlay. Thereafter, the presence of red fluorescent cells was analyzed at $72 \mathrm{~h}$ post infection using confocal and addition of methyl cellulose overlay. Thereafter, the presence of red fluorescent cells was analyzed at $72 \mathrm{~h}$ post infection using confocal
microscopy. (B) DI-244 supernatants produced in cells expressing PB2 or PB2opt were inoculated onto the indicated MDCK target cells as described for panel B and the presence of red fluorescent cells was analyzed at $72 \mathrm{~h}$ post infection using confocal microscopy. (C) The number of infected cells (as determined by red fluorescence) in panel A was quantified. In parallel, infection of cells was analyzed by focus formation assay and the number of foci was quantified. The results of a representative experiment are shown in panels A-C and were confirmed in two separate experiments. (D) The average of three (PB2) and six (PB2opt) independent experiments conducted as described for panel A is shown. Supernatants obtained at six days post transfection were analyzed, infection of cells was quantified by focus formation assay. Error bars indicate SEM.

https://doi.org/10.1371/journal.pone.0212757.g005

PB2 allow generation of infectious DI-244 particles solely from plasmids and in the absence of standard virus. Finally, our study shows that DIPs produced in this system suppress spread of different IAV subtypes but not VSV in cell culture.

DI-244 particles and other DIPs have so far been amplified in cell culture or hen's eggs in the presence of standard virus $[3,4,17]$. In addition, production of DI-244 particles from a plasmid system has been described $[34,35]$. This approach relies on the transfection of plasmids for production of infectious IAV in conjunction with a plasmid containing the DI-244 segment and results in the co-production of DIPs and standard virus [34, 35]. Before DIP preparations produced in these systems can be used for experimentation, the remaining standard virus needs to be inactivated by UV light [18]. This approach builds on the preferential inactivation of standard virus relative to DIPs. Thus, a mutation in a gene essential for viral spread will abrogate infectivity of standard virus but may have no effect on DIP infectivity since the missing proteins will be complemented in trans in cells coinfected with DIPs and standard virus. However, controlling the efficiency of UV inactivation of standard virus is technically challenging. Moreover, the effect of UV light on DIP infectivity is difficult to determine and both issues may complicate large scale production of DIPs as well as interpretation of experimental data and animal trials. Thus, establishment of novel cell culture systems for DIP production in the absence of standard virus is an important task.

Our results show that cell lines expressing PB2 allow production and quantification of DI244 particles solely from plasmids and in the absence of standard virus. This finding was not expected given that several reports indicate that PB2 expression alone is insufficient to allow robust spread of IAV variants with temperature sensitive mutations in the PB2 gene at nonpermissive temperatures $[20,21]$. Moreover, it has been suggested that PB2 expression might be associated with unwanted cytotoxic effects [4]. The present study suggests that up to $4 \times 10^{6}$ DI-244 particles/ml can be produced in cells expressing codon optimized PB2, which roughly translates into production of 10 infectious DIPs per cell, and it can be speculated that efficiency of DIP production can be further increased by employing cell lines stably coexpressing PB1, PB2 and PA. Occasionally, weak fluorescence has been observed in DIP inoculated control cells. This is most likely attributable to low levels of DI-244 mRNA expression facilitated by PB2 protein associated with DI-244 vRNA present in the infecting DIPs. In contrast, no evidence for production of infectious IAV due to recombination between the DI-244 RNA and the RNA encoding for PB2 was obtained, as judged by bright field microscopy, immunofluorescence, focus formation assay and RT-PCR analysis, indicating that the DIP production system reported here is safe.

Quantification of DIP production so far relied on quantitative RT-PCR and hemagglutination assay $[4,8,17]$, which do not provide information on particle infectivity. This limitation has been overcome by the present study which demonstrates that infectivity of DI-244 particles can be quantified using a standard technique, focus formation assay. The availability of this 


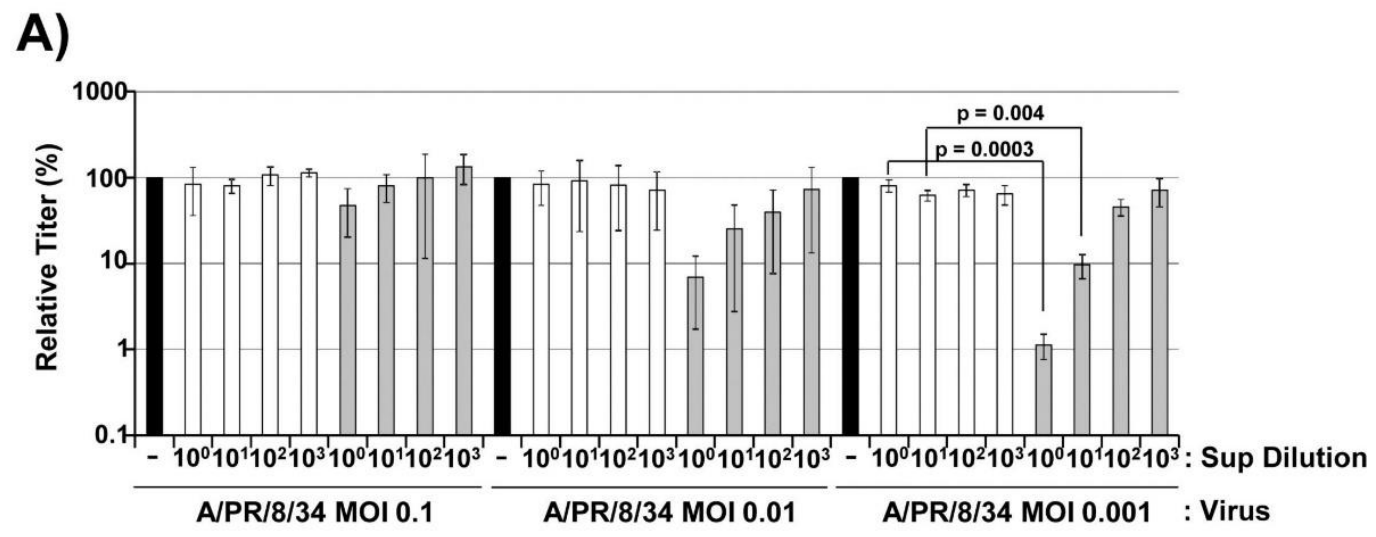

mock $\square$ Control Sups (MDCK-wt) $\square$ DIP Containing Sups (MDCK-PB2opt)

B)

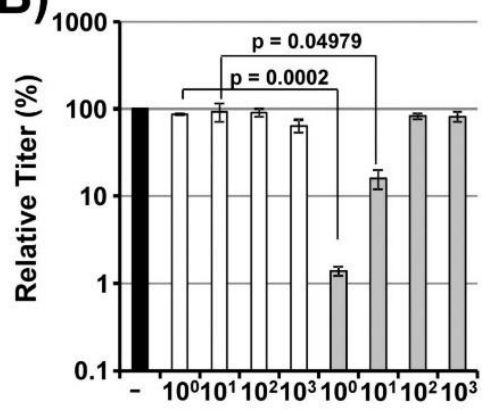

A/Panama/2007/99 MOI 0.001
C)

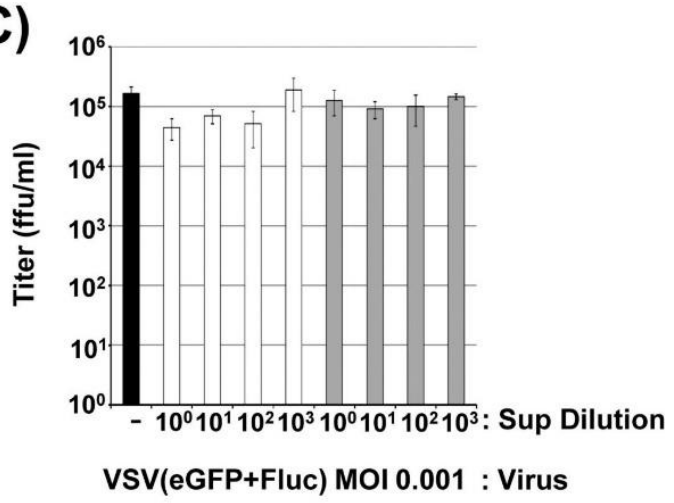

Fig 6. DI-244 produced in PB2opt expressing cell lines exerts anti-IAV activity. (A) Undiluted or 10-fold serially diluted DIP supernatants harvested from transfected 293T-PB2opt/MDCK-PB2opt cells or supernatants from transfected 293T/MDCK control cells were co-inoculated with A/PR/8/34 (H1N1) onto MDCK cells. Fresh medium was added at $1 \mathrm{~h}$ post infection and infectivity present in supernatants harvested at $72 \mathrm{~h}$ post infection was analyzed by focus formation assay. The average of three (MOI 0.1, MOI 0.01) and six (MOI 0.001), respectively, independent experiments is shown. Infection in the absence of supernatants was set as $100 \%$. Error bars indicate standard error of the mean (SEM). Two tailed paired students t-test was used to assess statistical significance. (B) The experiment was carried out as described for panel A but A/Panama/2007/99 (H3N2) was used for infection. The average of three independent experiments is shown. Infection in the absence of supernatants was set as $100 \%$. Error bars indicate SEM. Two tailed paired students t-test was used to assess statistical significance. (C) The experiment was carried out as for panel A but cells were infected with GFP-encoding VSV and supernatants were harvested for titration at $24 \mathrm{~h}$ post infection. The results of a single representative experiment conducted with triplicate samples are shown and were confirmed in two separate experiments.

https://doi.org/10.1371/journal.pone.0212757.g006

method should help comparing results obtained with different DI-244 preparations or other segment 1 DIPs and should thus advance the development of DIPs as antiviral agents. In this context, it is noteworthy that a IAV/DIP ratio of 1:1,000 resulted in the most prominent antiviral activity in our hands and a very similar ratio, 1:3,400 (as determined by estimations based on quantitative RT-PCR (DIP) and infectious units (IAV)), was previously reported to be 
minimally required to protect mice from severe influenza [4]. Thus, our study confirms and extends published work indicating that DIPs have to be provided in vast excess to exert antiviral activity. Whether sufficient numbers of DIPs can be delivered to the human respiratory tract and remain stable to provide protection against influenza for a prolonged time remains to be determined. In this context, one can speculate that an IAV:DIP ratio of less than 1:1,000 might be sufficient for antiviral activity in humans, since DIPs might exert direct antiviral activity by inhibiting IAV genome replication and induce the IFN system. Moreover, DIPs were reported to have a long residence time in the respiratory tract of mice and DIP-treated animals were found to still be protected at one week after treatment $[4,35]$. Thus, DIP stability in the respiratory tract might not pose a major hurdle to the use of DIPs for influenza prevention and therapy in humans. Finally, it should be stated that reassortment of DIPs with IAV in coinfected cells is likely to occur. However, if DIPs based on the low pathogenic A/PR/8/34 or related viruses are used (like in the present study), such reassortment events should not result in viruses with increased transmissibility or virulence as compared to the wt virus.

It is believed that DI-244 can interfere with spread of diverse IAV in cell culture due to genome competition [3, 4]. Indeed, DI-244 produced in PB2opt cells exerted comparable antiviral activity against $\mathrm{H} 1 \mathrm{~N} 1$ and $\mathrm{H} 3 \mathrm{~N} 2 \mathrm{IAV}$ (no statistically significant differences), in keeping with $\mathrm{H} 3 \mathrm{~N} 2$ polymerase complexes being fully functional on $\mathrm{H} 1 \mathrm{~N} 1$ genomic segments [36]. This matches data published for DI-244 generated by use of standard virus [35] and demonstrates that DIPs produced in PB2 expressing cells are fully functional, although the activity of purified DIPs remains to be examined. DI-244 can also interfere with the spread of influenza B virus (IBV) and unrelated respiratory viruses in the infected host and this is thought to be due to induction of innate immune responses, particularly the IFN response $[14,16]$. In contrast, DIP-mediated inhibition of IBV infection in cell culture is not observed, due to absence of genome competition $[13,14]$. The absence of antiviral activity of DIPs against VSV confirms lack of genome competition. Moreover, it suggests that DIPs might not have modulated a potential IFN response in MDCK cells, although it should be noted that such a response might have been impeded due to the presence of trypsin in the culture medium [37].

Collectively, we report, to our knowledge, the first experimental system for production of DIPs without standard virus and for quantification of DIP infectivity, which should promote efforts to develop DIPs for antiviral therapy.

\section{Supporting information}

S1 Fig. Alignment of PB2 and codon optimized PB2. The nucleotide sequences of PB2-wt (PB2) and codon optimized PB2 (PB2opt) were aligned using the Clustal W algorithm of AlignX (Vector NTI). Divergent nucleotides are marked in black. (PDF)

\section{Acknowledgments}

We thank Robert Webster for the 8-plasmid system for PR8 (pHW191-pHW198) and Georg Kochs and Martin Schwemmle for plasmids for the replicon assay and Defense Advanced Research Projects Agency (DARPA, Intercept Program) for support.

\section{Author Contributions}

Conceptualization: Stefan Pöhlmann, Michael Winkler.

Formal analysis: Markus Hoffmann, Stefan Pöhlmann, Michael Winkler. 
Funding acquisition: Stefan Pöhlmann.

Investigation: Najat Bdeir, Prerna Arora, Sabine Gärtner, Markus Hoffmann, Michael Winkler.

Resources: Udo Reichl.

Supervision: Stefan Pöhlmann, Michael Winkler.

Writing - original draft: Stefan Pöhlmann.

Writing - review \& editing: Najat Bdeir, Prerna Arora, Markus Hoffmann, Udo Reichl, Michael Winkler.

\section{References}

1. Paules C, Subbarao K. Influenza. Lancet. 2017; 390(10095):697-708. https://doi.org/10.1016/S01406736(17)30129-0 PMID: 28302313

2. Hussain M, Galvin HD, Haw TY, Nutsford AN, Husain M. Drug resistance in influenza A virus: the epidemiology and management. Infect Drug Resist. 2017; 10:121-34. https://doi.org/10.2147/IDR.S105473 PMID: 28458567

3. Dimmock NJ, Easton AJ. Defective interfering influenza virus RNAs: time to reevaluate their clinical potential as broad-spectrum antivirals? J Virol. 2014; 88(10):5217-27. https://doi.org/10.1128/JVI. 03193-13 PMID: 24574404

4. Dimmock NJ, Easton AJ. Cloned Defective Interfering Influenza RNA and a Possible Pan-Specific Treatment of Respiratory Virus Diseases. Viruses. 2015; 7(7):3768-88. https://doi.org/10.3390/ V7072796 PMID: 26184282

5. Zhao H, To KKW, Chu H, Ding Q, Zhao X, LiC, et al. Dual-functional peptide with defective interfering genes effectively protects mice against avian and seasonal influenza. Nat Commun. 2018; 9(1):2358. https://doi.org/10.1038/s41467-018-04792-7 PMID: 29907765

6. Smith CM, Scott PD, O'Callaghan C, Easton AJ, Dimmock NJ. A Defective Interfering Influenza RNA Inhibits Infectious Influenza Virus Replication in Human Respiratory Tract Cells: A Potential New Human Antiviral. Viruses. 2016; 8(8). https://doi.org/10.3390/v8080237 PMID: 27556481

7. Holland JJ, Doyle M. Attempts to detect homologous autointerference in vivo with influenza virus and vesicular stomatitis virus. Infect Immun. 1973; 7(4):526-31. PMID: 4357601

8. Frensing T, Heldt FS, Pflugmacher A, Behrendt I, Jordan I, Flockerzi D, et al. Continuous influenza virus production in cell culture shows a periodic accumulation of defective interfering particles. PLoS One. 2013; 8(9):e72288. https://doi.org/10.1371/journal.pone.0072288 PMID: 24039749

9. Dimmock NJ, Dove BK, Scott PD, Meng B, Taylor I, Cheung L, et al. Cloned defective interfering influenza virus protects ferrets from pandemic 2009 influenza $A$ virus and allows protective immunity to be enza virus protects ferrets from pandemic 2009 influenza A virus and allows protective immunity to
established. PLoS One. 2012; 7(12):e49394. https://doi.org/10.1371/journal. pone.0049394 PMID: 23251341

10. Rabinowitz SG, Huprikar J. The influence of defective-interfering particles of the PR-8 strain of influenza A virus on the pathogenesis of pulmonary infection in mice. J Infect Dis. 1979; 140(3):305-15. PMID: 227968

11. Mann A, Marriott AC, Balasingam S, Lambkin R, Oxford JS, Dimmock NJ. Interfering vaccine (defective interfering influenza $A$ virus) protects ferrets from influenza, and allows them to develop solid immunity to reinfection. Vaccine. 2006; 24(20):4290-6. https://doi.org/10.1016/j.vaccine.2006.03.004 PMID: 16621180

12. Dimmock NJ, Marriott AC. In vivo antiviral activity: defective interfering virus protects better against viruIent Influenza A virus than avirulent virus. J Gen Virol. 2006; 87(Pt 5):1259-65. https://doi.org/10.1099/ vir.0.81678-0 PMID: 16603528

13. Scott PD, Meng B, Marriott AC, Easton AJ, Dimmock NJ. Defective interfering virus protects elderly mice from influenza. Virol J. 2011; 8:212. https://doi.org/10.1186/1743-422X-8-212 PMID: 21549019

14. Easton AJ, Scott PD, Edworthy NL, Meng B, Marriott AC, Dimmock NJ. A novel broad-spectrum treatment for respiratory virus infections: influenza-based defective interfering virus provides protection against pneumovirus infection in vivo. Vaccine. 2011; 29(15):2777-84. https://doi.org/10.1016/j. vaccine.2011.01.102 PMID: 21320545

15. Scott PD, Meng B, Marriott AC, Easton AJ, Dimmock NJ. Defective interfering influenza virus confers only short-lived protection against influenza virus disease: evidence for a role for adaptive immunity in 
DI virus-mediated protection in vivo. Vaccine. 2011; 29(38):6584-91. https://doi.org/10.1016/j.vaccine. 2011.06.114 PMID: 21762748

16. Scott PD, Meng B, Marriott AC, Easton AJ, Dimmock NJ. Defective interfering influenza A virus protects in vivo against disease caused by a heterologous influenza B virus. J Gen Virol. 2011; 92(Pt 9):212232. https://doi.org/10.1099/vir.0.034132-0 PMID: 21632569

17. Wasik MA, Eichwald L, Genzel Y, Reichl U. Cell culture-based production of defective interfering particles for influenza antiviral therapy. Appl Microbiol Biotechnol. 2018; 102(3):1167-77. https://doi.org/10 1007/s00253-017-8660-3 PMID: 29204901

18. Nayak DP, Tobita K, Janda JM, Davis AR, De BK. Homologous interference mediated by defective interfering influenza virus derived from a temperature-sensitive mutant of influenza virus. J Virol. 1978 28(1):375-86. PMID: 702654

19. Ozawa M, Victor ST, Taft AS, Yamada S, Li C, Hatta M, et al. Replication-incompetent influenza A viruses that stably express a foreign gene. J Gen Virol. 2011; 92(Pt 12):2879-88. https://doi.org/10 1099/vir.0.037648-0 PMID: 21880840

20. Braam-Markson J, Jaudon C, Krug RM. Expression of a functional influenza viral cap-recognizing protein by using a bovine papilloma virus vector. Proc Natl Acad Sci U S A. 1985; 82(13):4326-30. Epub 1985/07/01. PMID: 2989815

21. Krystal M, Li R, Lyles D, Pavlakis G, Palese P. Expression of the three influenza virus polymerase proteins in a single cell allows growth complementation of viral mutants. Proc Natl Acad Sci U S A. 1986; 83(8):2709-13. Epub 1986/04/01. PMID: 3010315

22. Noble S, Dimmock NJ. Characterization of putative defective interfering (DI) A/WSN RNAs isolated from the lungs of mice protected from an otherwise lethal respiratory infection with influenza virus $A$ WSN (H1N1): a subset of the inoculum DI RNAs. Virology. 1995; 210(1):9-19. https://doi.org/10.1006/ viro. 1995.1312 PMID: 7793084

23. Hoffmann E, Krauss S, Perez D, Webby R, Webster RG. Eight-plasmid system for rapid generation of influenza virus vaccines. Vaccine. 2002; 20(25-26):3165-70. Epub 2002/08/07. PMID: 12163268

24. Gnirss K, Zmora P, Blazejewska P, Winkler M, Lins A, Nehlmeier I, et al. Tetherin Sensitivity of Influenza A Viruses Is Strain Specific: Role of Hemagglutinin and Neuraminidase. J Virol. 2015; 89 (18):9178-88. Epub 2015/06/26. https://doi.org/10.1128/JVI.00615-15 PMID: 26109730

25. Eckert N, Wrensch F, Gartner S, Palanisamy N, Goedecke U, Jager N, et al. Influenza A virus encoding secreted Gaussia luciferase as useful tool to analyze viral replication and its inhibition by antiviral compounds and cellular proteins. PLoS One. 2014; 9(5):e97695. Epub 2014/05/21. https://doi.org/10.1371/ journal.pone.0097695 PMID: 24842154

26. Bindels DS, Haarbosch L, van Weeren L, Postma M, Wiese KE, Mastop M, et al. $\mathrm{mScarlet:} \mathrm{a} \mathrm{bright}$ monomeric red fluorescent protein for cellular imaging. Nat Methods. 2017; 14(1):53-6. Epub 2016/11/ 22. https://doi.org/10.1038/nmeth.4074 PMID: 27869816

27. Gonzalez-Hernandez M, Hoffmann M, Brinkmann C, Nehls J, Winkler M, Schindler M, et al. A GXXXA Motif in the Transmembrane Domain of the Ebola Virus Glycoprotein Is Required for Tetherin Antagonism. J Virol. 2018; 92(13). Epub 2018/04/20. https://doi.org/10.1128/JVI.00403-18 PMID: 29669839

28. Wrensch F, Winkler M, Pohlmann S. IFITM proteins inhibit entry driven by the MERS-coronavirus spike protein: evidence for cholesterol-independent mechanisms. Viruses. 2014; 6(9):3683-98. Epub 2014/ 09/27. https://doi.org/10.3390/v6093683 PMID: 25256397

29. O'Doherty U, Swiggard WJ, Malim MH. Human immunodeficiency virus type 1 spinoculation enhances infection through virus binding. J Virol. 2000; 74(21):10074-80. Epub 2000/10/12. PMID: 11024136

30. Zimmermann $\mathrm{P}$, Manz B, Haller $\mathrm{O}$, Schwemmle M, Kochs $\mathrm{G}$. The viral nucleoprotein determines $\mathrm{Mx}$ sensitivity of influenza A viruses. J Virol. 2011; 85(16):8133-40. Epub 2011/06/18. https://doi.org/10. 1128/JVI.00712-11 PMID: 21680506

31. Schindelin J, Arganda-Carreras I, Frise E, Kaynig V, Longair M, Pietzsch T, et al. Fiij: an open-source platform for biological-image analysis. Nat Methods. 2012; 9(7):676-82. https://doi.org/10.1038/nmeth 2019 PMID: 22743772

32. Winkler M, Bertram S, Gnirss K, Nehlmeier I, Gawanbacht A, Kirchhoff F, et al. Influenza A virus does not encode a tetherin antagonist with Vpu-like activity and induces IFN-dependent tetherin expression in infected cells. PLoS One. 2012; 7(8):e43337. Epub 2012/09/07. https://doi.org/10.1371/journal.pone. 0043337 PMID: 22952667

33. Von Magnus P. Incomplete forms of influenza virus. Adv Virus Res. 1954; 2:59-79. Epub 1954/01/01. PMID: 13228257

34. Duhaut SD, Dimmock NJ. Defective segment 1 RNAs that interfere with production of infectious influenza A virus require at least 150 nucleotides of 5' sequence: evidence from a plasmid-driven system. $J$ 
Gen Virol. 2002; 83(Pt 2):403-11. Epub 2002/01/25. https://doi.org/10.1099/0022-1317-83-2-403 PMID: 11807233

35. Dimmock NJ, Rainsford EW, Scott PD, Marriott AC. Influenza virus protecting RNA: an effective prophylactic and therapeutic antiviral. J Virol. 2008; 82(17):8570-8. https://doi.org/10.1128/JVI.00743-08 PMID: 18579602

36. Phipps KL, Marshall N, Tao H, Danzy S, Onuoha N, Steel J, et al. Seasonal H3N2 and 2009 Pandemic H1N1 Influenza A Viruses Reassort Efficiently but Produce Attenuated Progeny. J Virol. 2017; 91(17) Epub 2017/06/24. https://doi.org/10.1128/JVI.00830-17 PMID: 28637755

37. Seitz C, Isken B, Heynisch B, Rettkowski M, Frensing T, Reichl U. Trypsin promotes efficient influenza vaccine production in MDCK cells by interfering with the antiviral host response. Appl Microbiol Biotechvaccine production in MDCK cells by interfering with the antiviral host response. Appl Micro
nol. 2012; 93(2):601-11. https://doi.org/10.1007/s00253-011-3569-8 PMID: 21915610 


\section{Second Manuscript}

Prerna Arora, Najat Bdeir, Sabine Gärtner, Stefanie Kramer, Lars Pelz, Ulrike Felgenhauer, Udo Reichl, Stephan Ludwig, Friedemann Weber, Gert Zimmer, Markus Hoffmann, Michael Winkler, Stefan Pöhlmann

Interferon induction and not replication interference is the major determinant of antiinfluenza virus activity of defective interfering particles

(Prepared for submission)

Individual contribution: In the following manuscript I performed experiments for Figure $1 \mathrm{~A}$, B, C, E and F; " Antiviral activity of DI-RNAs inversely correlates with DI RNA length in the presence of trypsin", Figure $2 \mathrm{~A}-\mathrm{E}$; "Induction of the IFN system is a major contributor to DIP antiviral activity" Figure 3A; "DI-244 does not induce IFN expression as determined in a VSVreplicon-based bioassay" Furthermore, I analysed the data for Figure 3B; "DI-244 induces robust ISG but not IFN expression as determined by RNAseq." 
1 Interferon induction and not replication interference is the major

2 determinant of anti-influenza virus activity of defective interfering particles

3

4

5

6

7

8

9

Prerna Arora, ${ }^{1,2}$ Najat Bdeir, ${ }^{1,2}$ Sabine Gärtner, ${ }^{1}$ Stefanie Kramer, ${ }^{1}$ Lars Pelz, ${ }^{3}$

\section{Ulrike Felgenhauer, ${ }^{6}$ Udo Reichl, ${ }^{3,4}$ Stephan Ludwig, ${ }^{5}$ Friedemann Weber, ${ }^{6}$ Gert}

\section{Zimmer, ${ }^{7}$ Markus Hoffmann, ${ }^{1}$ Michael Winkler, ${ }^{1}$ Stefan Pöhlmann,,${ }^{1,2,8^{*}}$}

${ }^{1}$ Infection Biology Unit, German Primate Center - Leibniz Institute for Primate Research, Göttingen, Germany

${ }^{2}$ Faculty of Biology and Psychology, University Göttingen, Göttingen, Germany

${ }^{3}$ Max Planck Institute for Dynamics of Complex Technical Systems, Magdeburg, Germany.

${ }^{4}$ Chair for Bioprocess Engineering, Otto-von-Guericke-University Magdeburg, Magdeburg, Germany

${ }^{5}$ Institute of Virology (IVM), University of Münster, 48149 Münster, Germany

${ }^{6}$ Institute for Virology, FB10 - Veterinary Medicine, Schubertstr. 81, D-35392 Gießen,

Germany

${ }^{7}$ Institute of Virology and Immunology, University of Bern Hochschulstrasse 6, 3012 Bern,

Switzerland

${ }^{8}$ Lead contact

${ }^{*}$ Correspondence 


\section{SUMMARY}

Influenza virus infection poses a serious threat to human health. Defective interfering (DI) RNAs result from errors during viral RNA replication and suppress influenza virus infection. DI RNAs packaged into defective interfering particles (DIPs) might allow for a novel approach to antiviral therapy. However, mutations required for converting a viral RNA into a DI RNA and the mechanism underlying DI RNA antiviral activity are incompletely understood. Here, we show that any central deletion is sufficient to convert a viral RNA into a DI RNA and that antiviral activity is inversely correlated with DI RNA length under conditions which disfavor inhibition of influenza virus infection by DIP-dependent induction of the IFN system. When full DIP-mediated induction of the IFN system was allowed before influenza virus infection, it was found to be the major contributor to DIP antiviral activity while DI RNA length played no detectable role. Notably, both DIPs and influenza virus triggered expression of IFN-stimulated genes (ISGs) while only virus stimulated robust expression of IFN, suggesting differences in DIP- and influenza virus-mediated activation of the effector functions of the IFN system. Collectively, our results support a model positing that DI RNAs inhibit viral infection by outcompeting wt RNAs for resources required for RNA replication but demonstrate that IFN induction outweighs replication interference in IFN-competent target cells.

4

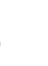

6

7




\section{INTRODUCTION}

The annually recurring influenza epidemics are a major source of global morbidity and mortality and intermittent pandemics can have even more severe consequences (Paules and Subbarao, 2017). Influenza therapy and vaccination are available but suffer from serious shortcomings (Paules and Subbarao, 2017). The success of influenza therapy with currently licensed drugs, which target the viral proteins neuraminidase (NA), matrix protein 2 (M2) or polymerase acidic protein (PA), can be compromised by resistance development (Han et al., 2018). Moreover, vaccines against epidemic influenza need to be annually adjusted to the viruses expected to circulate during the next influenza season and offer little or no protection against emerging pandemic viruses (Paules and Subbarao, 2017). Thus, the identification of novel targets and strategies for antiviral intervention is an important task.

Influenza viruses contain a segmented, negative sense RNA genome. The genomic segments are replicated by the viral polymerase, which consists of the subunits polymerase basic protein 1 (PB1), PB2 and PA (Te Velthuis and Fodor, 2016). The error rate of the viral polymerase is high and can result in the synthesis of genomic segments that harbor deletions (Davis et al., 1980; Davis and Nayak, 1979; Dimmock and Easton, 2014, 2015; Nakajima et al., 1979; Nayak et al., 1982; Nayak et al., 1978). Some of these defective segments interfere with the amplification of wt segments and are thus termed defective interfering (DI) RNAs (Davis et al., 1980; Davis and Nayak, 1979; Dimmock and Easton, 2014, 2015; Nakajima et al., 1979; Nayak et al., 1982). Packaging of DI RNAs into viral particles results in the formation of DI particles (DIPs), which suppress wt influenza virus spread (Dimmock and Easton, 2014, 2015). It has been proposed that DIPs suppress influenza virus infection by interfering with genome replication (a process subsequently termed replication interference) and by inducing interferon (IFN) (Baum et al., 2010; Dimmock and Easton, 2014, 2015; Frensing et al., 2014; Ngunjiri et al., 2013; Ngunjiri et al., 2012; Perez-Cidoncha et al., 2014; Scott et al., 2011a, b). However, this concept has not been systematically investigated and the 
relative contribution of replication interference and IFN induction to DIP antiviral activity is unknown.

We recently developed a cell culture system that allows production of genetically defined DIPs based on reverse genetics and a cell line complementing defects in influenza A virus (IAV) genomic segment 1 (Bdeir et al., 2019). Here, we used this system as well as a mini-replicon assay (Zimmermann et al., 2011) to analyze the contribution of replication interference and IFN induction to antiviral activity of DIPs. We report that in the minireplicon assay any central deletion in segment 1,2 or 3 converts these segments into DI RNAs, which suppress replication of diverse target segments. Inhibitory activity of these DI RNAs was inversely correlated with segment length and a similar correlation was seen in the context of DIPs and IAV infection under conditions which disfavored IAV inhibition by DIPdependent induction of the IFN system. If induction of the IFN system was allowed before IAV infection, it largely accounted for DIP antiviral activity. Finally, DIPs robustly induced ISG but not IFN expression, indicating that IAV and DIPs may differ in the activation of the effector functions of the IFN system. Our results suggest that although interference with genome replication contributes to DIP antiviral activity, the induction of IFN is the major determinant of suppression of virus infection by DIPs.

\section{RESULTS}

\section{DI-244 inhibits segment replication in a mini-replicon assay and inhibition is} independent of the truncated $\mathrm{PB} 2$ open reading frame

We first investigated whether a previously described IAV mini-replicon assay (Zimmermann et al., 2011) is suitable to detect inhibition of IAV genome replication by a prototypic segment 1-derived DI RNA, DI-244 (Dimmock et al., 2008). This assay is based on a firefly luciferase open reading frame flanked by the 5' and 3' ends of IAV segment 8, which is 
amplified in cells upon coexpression of the constituents of the viral polymerase complex, PB2, PB1, PA, and the viral NP protein (Zimmermann et al., 2011). Transfection of 293T cells with plasmids encoding the mini-genome reporter segment and the IAV proteins mentioned above resulted in luciferase activities in cell lysates that were approximately 1,000-fold higher than those measured in cells transfected with the reporter alone or transfected with the full set of plasmids except the PB2 encoding plasmid (Figure 1A). Moreover, cotransfection of two different amounts of DI-244 encoding plasmid resulted in a concentration dependent decrease in luciferase activity, indicating that DI-244 inhibited replication of the reporter segment (Figure 1A). This inhibitory activity was also observed when the PB2 start codon in DI-244 and two subsequent ATGs (positions 11 and 28) were mutated (Figure 1B). In contrast, transfection of expression plasmid pCAGGS containing the truncated PB2 ORF of DI-244 or empty pCAGGS did not reduce luciferase signals (Figure 1B). These results indicate that inhibition of segment replication by DI-244 can be visualized in the mini-replicon assay and does not require expression of truncated PB2.

Inhibitory activity of segment 1, 2 and 3-derived DI RNAs is inversely correlated with RNA length and is independent of the target segment

It is believed that the short length of DI-244 as compared to wt segment 1 results in faster amplification of DI-244 and ultimately in suppression of amplification of the wt segment (Dimmock and Easton, 2014, 2015). If correct, one would assume that the length of a DI RNA is a major determinant of antiviral activity. We explored this possibility by investigating the capacity of a set of ten segment 1-derived RNAs with nested central deletions to inhibit segment amplification in the mini-replicon assay. All RNAs tested exerted inhibitory activity and an inverse correlation between RNA length and inhibitory activity was observed (Figure 1C, Table S1). Moreover, further shortening of DI-244 did not augment inhibitory activity (not shown), suggesting that DI-244 length may be optimal for inhibition of wt segment 
130

131

132

133

134

135

136

137

138

139

140

141

142

143

144

145

146

147

148

149

150

151

152

153

154

155

replication. In sum, our results show that the ability of segment 1-derived DI RNAs to block replication of a wt segment is dependent on the DI RNA length.

We next explored whether the inverse correlation between length and inhibitory activity is also observed for segment 2- and 3-derived DI RNAs. For this, we introduced central, nested deletions in segment 2 and 3 and investigated inhibitory activity in the minireplicon system. As for segment 1-derived RNAs, all segment 2- and 3-based RNAs with deletions exerted inhibitory activity and inhibition inversely correlated with RNA length, although this correlation was more pronounced for segment 2 as compared to segment 3 (Figure 1D, Table S1).

Next, we examined whether the segment 1-, 2- and 3-derived DI RNAs with the largest deletion (constructs DI-244 (segment 1, S1), DI-156 (segment 2, S2), DI-178 (segment 3, S3), Table S1) were able to efficiently suppress replication of different IAV segments or were mainly active against segment 8 , which was so far employed in the mini-replicon assay. For this, we added the 5' and 3' ends of segments 1, 2, 4, 6, and 7 to the firefly luciferase sequence and tested the amplification of these reporter segments in the mini-replicon assay. In the absence of DI RNAs, all segments were efficiently amplified, as demonstrated by high luciferase activity in lysates of cells coexpressing PB2, PB1, PA and NP (Figure 1E). Cotransfection of two different amounts of segment 1-, 2- or 3-derived DI RNAs reduced replication of all reporter segments efficiently and in a concentration dependent manner (Figure 1E). Thus, in the mini-replicon assay, introduction of a deletion into an IAV genomic segment is sufficient to convert it into a DI RNA and length and inhibitory activity of these DI RNAs are inversely correlated.

\section{Inverse correlation between anti-IAV activity of DIPs and DI RNA length}

We recently reported a cell culture system for production of DIPs in the absence of helper virus, which relies on IAV reverse genetics and DIP producer cell lines stably expressing the 
156 PB2 protein (Bdeir et al., 2019). We employed this system to generate DIPs with nested deletions in segment 1 and assessed their ability to inhibit infection of MDCK cells with A/PR/8/34 (PR8). We found that DI-244, which contains the smallest DI RNA, inhibited PR8 infection with the highest efficiency and that inhibitory activity of DIPs decreased as DI RNA length increased (Figure 1F). Thus, an inverse correlation between DI RNA length and inhibitory activity observed in the mini-replicon assay could be confirmed in the context of DIPs, at least under the conditions tested.

\section{Preincubation of target cells with DI-244 increases antiviral activity}

It has been reported that DIPs can block viral infection by stimulating the IFN system (Scott et al., 2011a, b). Therefore, we sought to clarify whether induction of the IFN system could contribute to DI-244 antiviral activity in MDCK cells. Trypsin is used for A/PR8/34 activation but can inactivate IFNa (Figure 2A) (Seitz et al., 2012) and can thus cofound analyses of IAV inhibition by the IFN system. Therefore, we switched to A/WSN/33 (WSN) as challenge virus and WSN-derived DIPs, since WSN can replicate trypsin-independently in cell cultures containing fetal bovine serum (FBS) (Goto and Kawaoka, 1998). To obtain first insights into a potential role of the IFN system in DIP antiviral activity, we reasoned that if induction of the IFN system was a major determinant of DIP antiviral activity, then time-ofDIP addition to target cells should have a major impact on the efficiency of IAV inhibition by DIPs. Thus, addition of DIPs and virus to target cells at the same time should preclude the establishment of a DIP induced antiviral state prior to IAV infection. In contrast, addition of DIPs at $24 \mathrm{~h}$ before virus should allow for establishment of such an antiviral state and might thereby boost DIP antiviral activity. Preincubation of target MDCK cells with DI-244 for $24 \mathrm{~h}$ indeed increased DIP antiviral activity as compared to simultaneous addition of DI-244 and IAV, especially when high doses of DI-244 were analyzed (Figure 2B, left panel). Unexpectedly, similar results were obtained in the presence of trypsin (Figure 2B, right 
182

183

184

185

panel), indicating that the enhanced antiviral activity of DI-244 upon $24 \mathrm{~h}$ preincubation with target cells was likely not due to induction of IFN $\alpha$ or another trypsin-sensitive antiviral host cell protein.

\section{DI-244 induces anti-IAV activity in A549 cells in a STAT1-independent fashion}

In order to more directly assess the contribution of the IFN system to DI-244 antiviral activity, we used A549 wt cells and A549 cells which lack STAT1 (signal transducer and activator of transcription $1, \mathrm{STAT}^{-/}$) and are thus defective in IFN-induced signaling. Confirmatory experiments revealed that IFN $\alpha$, IAV and DI-244 strongly upregulated $M X 1$ expression in A549 wt but not $\mathrm{STAT1}^{-/-}$cells, in keeping with a defective JAK/STAT signaling pathway (Figure 2C). Addition of undiluted and 1:10 diluted DI-244 to A549 cells at $24 \mathrm{~h}$ before infection with WSN resulted in 100 -fold higher antiviral activity as compared to DI-244 added at the same time as virus (Figure 2D), confirming and extending the data obtained with MDCK cells. Unexpectedly, addition of undiluted DIP to A549 STAT1 ${ }^{-/-}$cells still resulted in high antiviral activity (Figure 2D), although 10-fold diluted DI-244 showed markedly reduced antiviral activity in $\mathrm{STAT}^{-/-}$cells as compared to wt cells. In contrast, inhibition of vesicular stomatitis virus (VSV) infection by DI-244 was completely dependent on STAT1, independent of the DIP dose used (Figure 2D). Finally, we asked whether the antiviral activity of DIPs still depends on the DI RNA length if DIPs are added to cells before virus. In contrast to what was observed with MDCK cells in the presence of trypsin, all DIPs with nested deletions in segment 1 inhibited WSN infection of A549 wt cells with similar efficiency (Figure 2E and data not shown), indicating that the contribution of replication interference to DIP antiviral activity was minor or absent under those conditions. Collectively, our findings indicate that DIPs can induce robust, partially STAT1-independent anti-IAV activity that is not determined by DI RNA length and markedly more potent than DIPmediated inhibition of IAV genome replication. 


\section{DI-244 induces robust expression of ISGs but not IFN}

209 In order to understand how DIPs, activate the IFN system, we compared DIP- and IAVmediated stimulation of IFN expression. For this, an IFN bioassay was employed that was based on VSV, a highly IFN-sensitive virus. A549 or A549 STAT1 $^{-/}$cells were incubated

212 with IAV, VSV or DI-244 for $24 \mathrm{~h}$, the supernatants collected and heat and acid treated to 213 inactivate viral particles but not IFN, which is known to display a certain heat and acid 214 stability. Subsequently, the supernatants were added to target cells for 16-18 h followed by 215 infection of A549 target cells with VSV and quantification of infection. For standardization, 216 A549 cells were incubated with recombinant IFN $\alpha$, infected and infection efficiency 217 quantified. Supernatants from IAV exposed A549 wt cells but not A549 STAT1 $^{-/}$cells 218 potently inhibited VSV infection (Figure 3A), indicating that IAV induced production of IFN 219 in a STAT1-dependent fashion, as expected. Similar findings were made with supernatants 220 from VSV exposed cells but antiviral activity was independent of STAT1 expression (Figure 221 3A), again in agreement with published data (Basu et al., 2006). Finally, and unexpectedly, 222 supernatants from A549 wt cells exposed to DI-244 were not inhibitory and the same finding 223 was made for supernatant from DI-244 treated A549 STAT1 $^{-/}$cells, indicating that IFN 224 induction by DI-244 was low or absent. 227 this question, A549 cells were either incubated with control supernatants or supernatants 228 containing DI-244 or IAV and subjected to RNAseq analysis. PR8 was employed for these 229 studies, in order to limit viral replication to a single cycle (since no trypsin was added). 230 Neither PR8 nor DI-244 induced the expression of IFN receptors (Figure 3B). In contrast, 231 PR8 but not DI-244 induced expression of IFN $\beta$ and IFN $\lambda$ (Figure 3B). Despite the 232 differential upregulation of IFNs by PR8 and DI-244 both induced the robust expression of 233 antiviral ISGs, including MX1, IFITM1 and ISG15, although induction by PR8 was more 
234 efficient than that observed for DI-244 (Figure 3B). Moreover, no ISG induction was 235 observed in PR8 or DIP treated A549 STAT1 $^{-/}$cells, with the exception of ISG15 and 236 RSAD2 (Viperin), the expression of which was induced by PR8 but not IAV. Finally, results 237 with A549 wt cells were confirmed by qRT-PCR analyses. Induction of IFN $\beta$ and IFN $\lambda$ by 238 DI-244 was at least 100-fold less efficient as compared to PR8 while differences in ISG 239 induction were frequently less than 10-fold (Figure 3C). In sum, these results suggest that DI244 inhibits viral infection by the IFN-independent, STAT1-dependent induction of ISG expression.

\section{DISCUSSION}

244 DI RNAs arise in IAV infected cell cultures, eggs, animals and patients (Bean et al., 1985; 245 Chambers and Webster, 1987; Dimmock and Easton, 2014, 2015; Dimmock et al., 2008; 246 Saira et al., 2013; Von Magnus, 1954). They inhibit IAV infection and might modulate IAV 247 intra- and interpatient spread and pathogenesis. However, the mechanism underlying DI RNA 248 antiviral activity and the determinants controlling whether a defective viral genomic RNA is 249 also interfering are incompletely understood. Here, we show that any central deletion in 250 segments 1, 2 and 3 of IAV is sufficient to convert these RNAs into DI RNAs and that 251 inhibitory activity of the respective DI RNAs extends to all tested IAV genomic RNAs. 252 Moreover, we provide evidence that the contribution of replication interference to DIP 253 antiviral activity in cell culture is minor as compared to induction of the IFN system. IAV and influenza B virus DI RNAs usually contain deletions relative to the genomic 255 RNAs they arose from (Dimmock and Easton, 2014, 2015), although an exception has 256 recently been reported (Kupke et al., 2019). Moreover, DI RNAs derived from IAV segments 257 1-3, which encode the subunits of the viral polymerase, arise more frequently than those 258 derived from other segments (Davis et al., 1980; Davis and Nayak, 1979; Dimmock and 259 Easton, 2014, 2015; Frensing et al., 2013; Moss and Brownlee, 1981; Nakajima et al., 1979) 
and were thus in the focus of the present study. The almost universal presence of a deletion in DI RNAs suggests that their shorter length might allow them to out-compete their parental RNAs for resources required for RNA replication. Although this hypothesis is frequently posited (Dimmock and Easton, 2014, 2015), direct experimental proof is largely lacking. Here we provide this proof by demonstrating that deleting any internal sequence from segments 1 , 2 and 3 is sufficient to generate a DI RNA. Furthermore, we demonstrate that the inhibitory activity of these DI RNAs is determined by their length, at least in the absence of an IFN response, and extends to all target segments tested. The latter observation fits with the finding that DI-244 interferes with replication of several genomic RNAs in IAV infected cells (Meng et al., 2017). In sum, deleting the sequences between the conserved 5' and 3' ends of any IAV RNA, which are required for transcription and translation, should generate potent DI RNAs. In some cases, the truncated open reading frame encoded by such DI RNAs might contribute to antiviral activity (Boergeling et al., 2015) but this was not observed for DI-244, in keeping with previous results (Meng et al., 2017).

Type I IFN triggers the expression of about 400 genes, many of which encode proteins with antiviral activity, including MX1 (Schoggins et al., 2011). The present study shows that when conditions are chosen that allow DIPs to activate the IFN system, DIPs are potent inducers of ISG expression and the contribution of replication interference to DIP antiviral activity is minor. Notably, RNAseq analysis revealed that IAV but not DIPs robustly induced type I and type III IFN expression although both triggered ISG expression in a STAT1dependent fashion. A potential explanation for this discrepancy is that DIPs induced IFN expression at levels too low to be detected by RNAseq but still sufficient to induce ISGs. Indeed, qRT-PCR analysis revealed modest upregulation of type I and III IFN upon DIP treatment. Alternatively, DIPs may induce ISGs via an unknown IFN-independent, STAT1dependent pathway. Interestingly, Wang and colleagues also reported that DIPs induce robust levels of ISGs but not IFN (Wang et al., 2020) and further research is required to explore the 
underlying reasons. Moreover, it is unclear how undiluted DIPs exerted anti-IAV but not anti-

VSV activity in STAT1 ${ }^{-/-}$cells without inducing ISGs or other cellular genes. Collectively, our results underline previous findings that DIPs are potent IFN inducers (Baum et al., 2010; and show that DIP antiviral activity due to IFN induction outweighs that due to replication interference.

What are the major implications of our findings for DIP development as antivirals and for elucidating the role of naturally occurring DIPs in IAV infection? First, it is essential that antiviral activity of DIPs is examined in IFN competent animal models which express ISGs with potent anti-IAV activity, particularly MX1. Second, antiviral activity due to replication interference can be attained only if DIPs are added in 100 to 1,000 -fold excess relative to virus (Bdeir et al., 2019) and it remains to be examined whether the strong IFN induction under those conditions exerts unwanted toxic effects in animals and humans. Third, DIP treatment should be more effective in the prophylactic as compared to the therapeutic setting, since only in the former DIP-induced IFN can fully contribute to antiviral activity. Fourth, design of DI RNA and analysis of DI RNAs emerging in patients should focus on the smallest 
MATERIAL AND METHODS

313

\section{Plasmids and oligonucleotides}

315

Plasmids for rescue of A/PR/8/34, pHW191-pHW198, and A/WSN/33, pHW181-pHW188, were previously described (Hoffmann et al., 2002). Plasmids encoding DI RNAs were generated by splice overlap PCR, joining 5' and 3'-end sequences of desired length, following a strategy previously described for DI-244 (Bdeir et al., 2019). A multiple cloning site (mcs) for later insertion of a reporter gene was included in the respective oligonucleotide sequences (Table S2). The PCR products were cloned into pHW2000-GGAarI by golden gate cloning (Eckert et al., 2014). Start codons in DI-244 were mutated using splice overlap PCR primer pairs mutIAV-seg1-ATG-for (5'- TCAATTATATTCAATTTGGAAAGAATAAAAG 3')/mutIAV-seg1-ATG-rev (5'- CTTTTATTCTTTCCAAATTGAATATAATTGA-3') and DImut2+3ATG-for (5' ACTACGAAATCTAATCTCGCAGTCTCGCACCCGCGAGATAC TCACAAAAACCACCGTGGACCATATCGCCATAATCAAGAAG-3')/DImut2+3ATGrev (5'-CTTCTTGATTATGGCGATATGGTCCACGGTGGTTTTTGTGAGTATCTCGCG GGTGCGAGACTGCGAGATTAGATTTCGTAGT-3'). PCR constructs were cloned into pHW2000-GGAarI as described above.

For expression of the truncated PB2 ORF from DI-244, the ORF was amplified from pHW2000GG-DI244-rep using primers PB2-QCXIP-5N (5'- CCGCGGCCGCACCATGGA AAGAATAAAAGAACTAC-3’)/PB2-3XBgl (5'-GGAGATCTCGAGCTAATTGATGGCC ATCCGAAT-3') digested with NotI/XhoI and cloned into NotI/SalI digested pCAGGS-mcs bearing an altered multiple cloning site (XhoI-SacI-Asp718I-NotI-EcoRV-ClaI-EcoRI-SmaISalI-SphI-NheI-BglII).

For generation of empty vector p19polI-GGAarI the insert was amplified from pHW2000GGAarI by splice overlap PCR using primers HW2-GG-5Bgl, CCdelE-rev (5'CGTCTTTCATTGCCATACGAAACTCCGGATGAGCATTCATCAG-3'), CCdelE-for (5'- 
CTGATGAATGCTCATCCGGAGTTTCGTATGGCAATGAAAGACG-3’’)/ rRNA-Pr(GG)-

339

3Eco (5'-GCGAATTCTATAGAATAGGGCCAGGTC-3') and cut with BglII and EcoRI for insertion into BamHI and EcoRI digested p19luc (Winkler et al., 1994).

Reporter plasmids for mini-replicon assay have been described (pPolI-Luc (vRNA/FLUAV/NS1 Seg8-NCR) (Zimmermann et al., 2011) or were newly generated. First, the reporter with segment 8 ends was amplified with primers fluA AarI-NS-1 and fluA AarINS-890R (Table S3) and inserted into vector p19polI-GGAarI by Golden Gate cloning. To generate reporters with ends derived from other segments of IAV, the luciferase reporter gene was amplified with primers encoding the respective untranslated regions (Table S3) and cloned into vector p19polI-GGAarI as described before. All PCR amplified sequences were confirmed by automated sequence analysis.

\section{Cells and viruses}

293T, A549 wt and A549 STAT1 $^{-/}$cells were maintained in Dulbecco's Modified Eagle Medium (DMEM; Gibco) containing 10\% fetal bovine serum (FBS, Gibco) and penicillin (Pen, $100 \mathrm{IU} / \mathrm{mL}$ ) and streptomycin (Strep, $100 \mu \mathrm{g} / \mathrm{ml}$ ). BHK-21 cells were cultivated in Dulbecco's modified Eagle medium (DMEM, Pan Biotech) supplemented with 5\% fetal bovine serum and pen/strep. $293 \mathrm{~T}$ cell lines stably expressing codon optimized PB2 (293TPB2opt) were cultured in the presence of $1 \mu \mathrm{g} / \mathrm{ml}$ puromycin. Madin-Darby canine kidney cells (MDCK) were cultured in Glasgow's Modified Eagle Medium (GMEM; Gibco) supplemented with $10 \%$ fetal bovine serum (FBS, Gibco) and pen/strep. MDCK cells stably expressing PB2opt were maintained in the presence of $1.5 \mu \mathrm{g} / \mathrm{ml}$ puromycin. For generation of A549 STAT1 $^{-/}$cells, A549 wt cells were transduced with a commercially available lentivirus expressing Cas9 (Addgene, plasmid 52961), puromycin resistance, and a guide RNA targeting human STAT1 (TTCAAGACCAGCGGCCTCTGAGG). Transduced cells were puromycin selected for seven days and surviving cells were plated in 96-well dishes as 
single cells and expanded. Clonal populations were then lysed and whole cell extract was examined for STAT1 expression by Western blot. These efforts identified a single clone that demonstrated a complete loss of STAT1 expression, which we refer herein as STAT1 ${ }^{-/}$cells. All cells lines were regularly tested for mycoplasma contamination.

A/PR/8/34 (H1N1) and A/WSN/33 (H1N1) (Hoffmann et al., 2002) were produced in embryonated chicken eggs as described previously (Zmora et al., 2017) while A/WSN/33 adapted to growth in A549 cells was obtained from the strain repository of the IVM Münster and was amplified in A549 cells by continuous passaging. IAV titers were determined using focus formation assay as described (Eckert et al., 2014; Winkler et al., 2012). Replicationcompetent vesicular stomatitis virus (VSV) expressing eGFP and either wildtype VSV matrix protein $\left(\mathrm{VSV}^{*}\right)$ or a matrix protein variant harboring four amino acid substitutions associated with increased induction of type-I interferon response $\left(\mathrm{VSV}^{*} \mathrm{M}_{\mathrm{Q}}\right)$ have been described elsewhere (Hoffmann et al., 2010) and were amplified using BHK-21. Further, a VSV glycoprotein trans-complemented, single-cycle VSV replicon that lacks the genetic information for VSV-G but instead codes for eGFP and firefly luciferase genes (VSV* $\Delta \mathrm{G}-$ FLuc) (Berger Rentsch and Zimmer, 2011) was employed and propagated on BHK-G43 cells (Hanika et al., 2005). All VSV variants were titrated on BHK-21 cells and eGFP-positive foci (replication-competent VSV) or eGFP-positive single cells (single-cycle VSV) were counted.

\section{Mini-replicon assay}

The mini-replicon assay was performed as described (Zimmermann et al., 2011). In brief, $293 \mathrm{~T}$ cells seeded in 12 -well plates at a cell density of $2 \times 10^{5}$ cells per well were cotransfected with plasmids encoding PB1 (10 ng), PB2 (10 ng), NP (100 ng), reporter segment encoding firefly luciferase (50 ng) and plasmid encoding a DI RNA or empty plasmid (amounts indicated in figures or figure legends). Cells were washed at 6-8 $\mathrm{h}$ and harvested at $24 \mathrm{~h}$ post transfection. Firefly luciferase activity in cell lysates was measured 
390

using a commercial kit (PJK) and the Plate Chameleon V reader (Hidex) jointly with Microwin 2000 software.

\section{Production of DIPs}

A coculture of $1.4 \times 10^{6} 293 \mathrm{~T}$ cells and $0.4 \times 10^{6}$ MDCK cells stably expressing PB2opt and seeded in T-25 flask was cotransfected with plasmids encoding IAV genomic segments 2-8 of either PR8 or WSN origin and a plasmid encoding a segment 1-derived DI-RNA. After overnight incubation, cells were washed once with PBS and, for production of $\mathrm{A} / \mathrm{PR} / 8 / 34$ derived DIPs, DMEM infection medium (0.2\% MACS BSA, 1\% pen/strep) supplemented with TPCK trypsin $(0.5 \mu \mathrm{g} / \mathrm{ml})$ was added. For production of A/WSN/33-derived DIPs, DMEM growth medium (2\% FCS, $1 \%$ pen/strep) was added. As a negative control, parental MDCK and 293T cells were transfected. Supernatants containing A/PR/8/34-derived DIPs were harvested at $4,6,8$ and 10 days post transfection while supernatants containing A/WSN/33-derived DIPs were harvested at 3, 5, 7 and 9 days post transfection. Supernatants were cleared from debris by centrifugation, aliquoted and stored at $-80{ }^{\circ} \mathrm{C}$ for further use. For some experiments, DIPs were further amplified in MDCK-PB2opt cells. For this, a total of 3 $\times 10^{6}$ cells were seeded in T-75 flasks and infected at an MOI of 0.01 or lower. Upon detection of CPE, supernatants were cleared from debris by centrifugation and sterile-filtration $(0.45$ $\mu \mathrm{m}$ filter), aliquoted and stored at $-80{ }^{\circ} \mathrm{C}$ for further use. Integrity of selected DIP preparations was controlled with segments specific PCR. Infectious titers of supernatants were determined by focus formation assay using MDCK-PB2opt cells as targets, as described (Bdeir et al., 2019; Eckert et al., 2014; Winkler et al., 2012).

\section{Analysis of antiviral activity of DIPs}

For testing the antiviral activity of DIPs in MDCK cells in the presence of trypsin, cells were seeded at 10,000 cells/well in 96-well plates and coinfected with DIP (MOI 1, and 10-fold 
dilutions) and IAV (A/PR/8/34, MOI 0.001) for $1 \mathrm{~h}$ in Glasgow's MEM (GMEM) infection medium containing trypsin $(0.5 \mu \mathrm{g} / \mathrm{ml})$. Alternatively, DIPs were added $24 \mathrm{~h}$ prior to the virus. For analysis of DIP antiviral activity in MDCK cells, A549 wt and A549 STAT $^{-/-}$cells in the absence of trypsin, cells were again seeded at 10,000 cells/well in 96-well plates and either coinfected with DIP (MOI 5 or 10, and 10-fold dilutions) and IAV (A/WSN/33, MOI 0.1) in DMEM medium without trypsin or DIPs added $24 \mathrm{~h}$ prior to the virus. After $1 \mathrm{~h}$, cells coexposed to DIPs and virus were washed and culture medium with or without trypsin was added. Supernatants were harvested after 72 h (MDCK) and 96 h (A549 wt and A549 STAT1${ }^{\circ}$ ). Viral titers in culture supernatants were quantified using focus formation assay and MDCK cells, as described (Eckert et al., 2014; Winkler et al., 2012).

\section{Quantitative RT-PCR analysis}

In order to investigate modulation of $M X 1 \mathrm{mRNA}$ expression by IAV, DIPs and IFN, a quantitative RT-PCR assay was performed. For this, A549 cells were seeded at a cell density of $2 \times 10^{5}$ cells/well in 12-well plates and inoculated with IAV (MOI 1), DIPs (MOI 1) or panIFNa (100 U/ml, PBL Assay Science) using DMEM infection medium for $1 \mathrm{~h}$ (DMEM infection medium without trypsin was added to cells exposed to IFN $\alpha$ ). Then cells were washed once with PBS and cultured in DMEM infection medium without trypsin for $24 \mathrm{~h}$. To assess the effect of trypsin on $M X 1$ induction by IFNa, cells were incubated for $24 \mathrm{~h}$ with IFN $\alpha$ in the presence of $0,0.5,0.05$ and $0.005 \mu \mathrm{g} / \mathrm{ml}$ trypsin. At $24 \mathrm{~h}$ post treatment, total cellular RNA was extracted using the RNeasy Mini kit (Qiagen) following the manufacturer's instructions. After determining the RNA content, $1 \mu \mathrm{g}$ RNA was used as template for cDNA synthesis employing the SuperScript III First-Strand Synthesis System (ThermoFisher Scientific), following the protocol for random hexamers. Subsequently, $1 \mu 1$ of cDNA (total volume after cDNA synthesis: $20 \mu \mathrm{l}$ ) was analyzed by quantitative PCR on a Rotorgene Q device (Qiagen) employing the QuantiTect SYBR Green PCR Kit (Qiagen). Each sample was 
442

443

444

445

446

447

448

449

450

451

452

453

454

455

456

457

458

459

460

461

462

463

464

465

analyzed in triplicates for transcript levels - given as cycle threshold $(\mathrm{Ct})$ values - of $\beta$-actin $(A C T B$, internal transcript control) and myxovirus resistance protein 1 ( $M X 1$, indicator for IFN induction, target transcript) with primers previously reported by Biesold and colleagues (Biesold et al., 2011). In order to analyze the gene expression, the $2^{-\Delta \Delta \mathrm{Ct}}$ method was used (Livak and Schmittgen, 2001).

\section{Vesicular Stomatitis Virus Replicon-Based Bioassay}

To analyze the relative contribution of IFN induction to antiviral activity, a VSV repliconbased bioassay was performed. This assay is based on the principle that inoculation of effector cells with virus or DIPs leads to the induction of the innate immune system, resulting in the release of type-I IFN into the culture supernatant. These supernatants are then used to inoculate sentinel cells. Here, the type-I IFN will bind to the IFN $\alpha / \beta$ receptors and trigger a signal cascade leading to the induction of an antiviral state. Subsequent inoculation of the sentinel cells with a highly IFN-sensitive VSV replicon containing a luciferase reporter will yield luciferase activities that inversely correlate with the extent of the induced antiviral state. A549 and A549 STAT1 $^{-/-}$cells (= effector cells) were seeded in 12-well plate $(200,000$ cells/well) and inoculated with IAV, VSV*, VSV*-MQ or DIPs (all at MOI of 1) using DMEM infection medium containing trypsin for $1 \mathrm{~h}$. The cells were washed once with PBS and cultured in DMEM infection medium without trypsin (used for all further steps) for 16-18 hours. Next, supernatant was harvested, and infectious virus was inactivated by addition of $0.1 \mathrm{M} \mathrm{HCl}$ and heating the samples for 30 mins to $56{ }^{\circ} \mathrm{C}$. After the samples cooled down to room temperature, alkaline treatment was performed using $0.1 \mathrm{M} \mathrm{NaOH}$ to neutralize the acidic $\mathrm{pH}$. Subsequently, the two-fold serial dilutions of the samples were prepared. In addition, medium containing two-fold serial dilutions of recombinant pan IFN $\alpha$ (starting at a concentration of $400 \mathrm{U} / \mathrm{ml}$ ) were treated in the same fashion. These samples served as reference and were used to calculate the relative antiviral activity present in the different 
468 supernatants (given as relative IFN $\alpha$ units per $\mathrm{ml}$ ). The diluted supernatants and IFN $\alpha$

469 reference samples were added in quadruplicates to a confluent layer of A549 cells grown in

470 96-well plates (= sentinel cells) and incubated for 18-24 h. Thereafter, the cells were

471 inoculated with $\mathrm{VSV}^{*} \Delta \mathrm{G}-\mathrm{FLuc}$ reporter virus (MOI of 3) and further incubated for $6 \mathrm{~h}$. Then,

472 the medium was aspirated and $50 \mu \mathrm{l} /$ well of $1 \mathrm{x}$ luciferase lysis buffer was added. Following

473 an incubation period of $30 \mathrm{~min}$, the lysates were transferred into white, opaque-walled 96-

474 well plates and firefly luciferase activity was measured as described above for the mini-

475 replicon assay. For normalization, luciferase activity was set as $100 \%$ for cells that received

476 regular culture medium instead of diluted culture supernatant/IFN $\alpha$ prior to inoculation with

$477 \mathrm{VSV}^{*} \Delta \mathrm{G}$-FLuc. Using the normalized luciferase values of cells treated with the IFN $\alpha$

478 reference samples and a non-linear regression model we then calculated the relative IFN $\alpha$

479 content (given as units per $\mathrm{ml}$ ) for the effector cell supernatants.

\section{RNA-seq analysis}

482 For analysis of IAV and DIP mediated modulation of cellular gene expression, A549 wt and

483 A549 STAT1 ${ }^{-/}$cells were exposed to A/PR/8/34, DI-244 or control supernatants at a MOI of 484 1. At $24 \mathrm{~h}$ post treatment, total cellular RNA was extracted using the RNeasy Mini kit 485 (Qiagen) following the manufacturer's instructions and subsequently sent for RNAseq 486 analysis at the Integrative Genomics Core Unit (NIG), Department of Human Genetics, 487 University Medical Center Göttingen.

488 RNA-seq libraries were performed using the non-stranded mRNA Kit (Illumina). Quality and 489 integrity of RNA was assessed with the Fragment Analyzer using the standard sensitivity 490 RNA Analysis Kit (Advanced Analytical). All samples selected for sequencing exhibited an 491 RNA integrity number of $>8$. After library generation, we used the QuantiFluor ${ }^{\mathrm{TM}} \mathrm{dsDNA}$ 492 System (Promega) for accurate quantitation of cDNA libraries. The size of final cDNA 493 libraries was determined by using the dsDNA 905 Reagent Kit (Advanced Analytical) 
exhibiting a sizing of $300 \mathrm{bp}$ in average. Libraries were pooled and sequenced on an Illumina

495 HiSeq 4000 (Illumina) generating 50 bp single-end reads (28-35 Mio reads/sample). The raw

496 read \& quality check were done by transforming sequence images with the BaseCaller 497 software (Illumina) to BCL files, which were demultiplexed to fastq files with bcl2fastq 498 v2.20. The sequencing quality was asserted using FastQC 499 (http://www.bioinformatics.babraham.ac.uk/projects/fastqc/).

500 For subsequent data analysis, ISGs with anti-IAV activity were selected based on work by 501 Schoggins and colleagues (Schoggins et al., 2011). ISG expression in IAV- or DIP-treated 502 cells is shown relative to expression of the same ISGs in control-treated cells.

503

504

505

506

507

508

509

510

511

512

513

514

515

516

517

518

\section{SUPPLEMENTAL INFORMATION}

Table S1, Table S2, Table S3

\section{ACKNOWLEDGEMENTS}

We would like to thank Benjamin TenOever and Martin Schwemmle for the kind gift of A549 $\mathrm{STAT}^{-/-}$cells and minireplicon plasmids, respectively, and DARPA (Intercept program) for funding.

\section{AUTHOR CONTRIBUTIONS}

P.A., N.B., S.G., S.K., L.P. and U.F. conducted experiments, M.W., M.H., F.W. and S.P. designed experiments and analyzed data, U.R., S.L. and G.Z. contributed vital reagents, S.P. wrote the manuscript. 


\section{REFERENCES}

Basu, M., Maitra, R.K., Xiang, Y., Meng, X., Banerjee, A.K., and Bose, S. (2006). Inhibition of vesicular stomatitis virus infection in epithelial cells by alpha interferon-induced soluble secreted proteins. J Gen Virol 87, 2653-2662.

Baum, A., Sachidanandam, R., and Garcia-Sastre, A. (2010). Preference of RIG-I for short viral RNA molecules in infected cells revealed by next-generation sequencing. Proc Natl Acad Sci U S A 107, 16303-16308.

Bdeir, N., Arora, P., Gartner, S., Hoffmann, M., Reichl, U., Pohlmann, S., and Winkler, M. (2019). A system for production of defective interfering particles in the absence of infectious influenza A virus. PLoS One 14, e0212757.

Bean, W.J., Kawaoka, Y., Wood, J.M., Pearson, J.E., and Webster, R.G. (1985). Characterization of virulent and avirulent $\mathrm{A} /$ chicken/Pennsylvania/83 influenza $\mathrm{A}$ viruses: potential role of defective interfering RNAs in nature. J Virol 54, 151-160.

Berger Rentsch, M., and Zimmer, G. (2011). A vesicular stomatitis virus replicon-based bioassay for the rapid and sensitive determination of multi-species type I interferon. PLoS One 6, e25858.

Biesold, S.E., Ritz, D., Gloza-Rausch, F., Wollny, R., Drexler, J.F., Corman, V.M., Kalko, E.K., Oppong, S., Drosten, C., and Muller, M.A. (2011). Type I interferon reaction to viral infection in interferon-competent, immortalized cell lines from the African fruit bat Eidolon helvum. PLoS One 6, e28131.

Boergeling, Y., Rozhdestvensky, T.S., Schmolke, M., Resa-Infante, P., Robeck, T., Randau, G., Wolff, T., Gabriel, G., Brosius, J., and Ludwig, S. (2015). Evidence for a Novel Mechanism of Influenza Virus-Induced Type I Interferon Expression by a Defective RNAEncoded Protein. PLoS Pathog 11, e1004924.

Chambers, T.M., and Webster, R.G. (1987). Defective interfering virus associated with A/Chicken/Pennsylvania/83 influenza virus. J Virol 61, 1517-1523.

Davis, A.R., Hiti, A.L., and Nayak, D.P. (1980). Influenza defective interfering viral RNA is formed by internal deletion of genomic RNA. Proc Natl Acad Sci U S A 77, 215-219.

Davis, A.R., and Nayak, D.P. (1979). Sequence relationships among defective interfering influenza viral RNAs. Proc Natl Acad Sci U S A 76, 3092-3096.

Dimmock, N.J., and Easton, A.J. (2014). Defective interfering influenza virus RNAs: time to reevaluate their clinical potential as broad-spectrum antivirals? J Virol 88, 5217-5227.

Dimmock, N.J., and Easton, A.J. (2015). Cloned Defective Interfering Influenza RNA and a Possible Pan-Specific Treatment of Respiratory Virus Diseases. Viruses 7, 3768-3788.

Dimmock, N.J., Rainsford, E.W., Scott, P.D., and Marriott, A.C. (2008). Influenza virus protecting RNA: an effective prophylactic and therapeutic antiviral. J Virol 82, 8570-8578.

Eckert, N., Wrensch, F., Gartner, S., Palanisamy, N., Goedecke, U., Jager, N., Pohlmann, S., and Winkler, M. (2014). Influenza A virus encoding secreted Gaussia luciferase as useful tool 
to analyze viral replication and its inhibition by antiviral compounds and cellular proteins. PLoS One 9, e97695.

Frensing, T., Heldt, F.S., Pflugmacher, A., Behrendt, I., Jordan, I., Flockerzi, D., Genzel, Y., and Reichl, U. (2013). Continuous influenza virus production in cell culture shows a periodic accumulation of defective interfering particles. PLoS One 8 , e72288.

Frensing, T., Pflugmacher, A., Bachmann, M., Peschel, B., and Reichl, U. (2014). Impact of defective interfering particles on virus replication and antiviral host response in cell culturebased influenza vaccine production. Appl Microbiol Biotechnol 98, 8999-9008.

Goto, H., and Kawaoka, Y. (1998). A novel mechanism for the acquisition of virulence by a human influenza A virus. Proc Natl Acad Sci U S A 95, 10224-10228.

Han, J., Perez, J., Schafer, A., Cheng, H., Peet, N., Rong, L., and Manicassamy, B. (2018). Influenza Virus: Small Molecule Therapeutics and Mechanisms of Antiviral Resistance. Curr Med Chem 25, 5115-5127.

Hanika, A., Larisch, B., Steinmann, E., Schwegmann-Weßels, C., Herrler, G., \& Zimmer, G. (2005). Use of influenza $C$ virus glycoprotein HEF for generation of vesicular stomatitis virus pseudotypes. The Journal of general virology, 86(Pt 5), 1455-1465.

Hoffmann, E., Krauss, S., Perez, D., Webby, R., and Webster, R.G. (2002). Eight-plasmid system for rapid generation of influenza virus vaccines. Vaccine 20,3165-3170.

Hoffmann, M., Wu, Y.J., Gerber, M., Berger-Rentsch, M., Heimrich, B., Schwemmle, M., and Zimmer, G. (2010). Fusion-active glycoprotein G mediates the cytotoxicity of vesicular stomatitis virus M mutants lacking host shut-off activity. J Gen Virol 91, 2782-2793.

Kupke, S.Y., Riedel, D., Frensing, T., Zmora, P., and Reichl, U. (2019). A Novel Type of Influenza A Virus-Derived Defective Interfering Particle with Nucleotide Substitutions in Its Genome. J Virol 93.

Livak, K.J., and Schmittgen, T.D. (2001). Analysis of relative gene expression data using real-time quantitative PCR and the 2(-Delta Delta C(T)) Method. Methods 25, 402-408.

Meng, B., Bentley, K., Marriott, A.C., Scott, P.D., Dimmock, N.J., and Easton, A.J. (2017). Unexpected complexity in the interference activity of a cloned influenza defective interfering RNA. Virol J 14, 138.

Moss, B.A., and Brownlee, G.G. (1981). Sequence of DNA complementary to a small RNA segment of influenza virus A/NT/60/68. Nucleic Acids Res 9, 1941-1947.

Nakajima, K., Ueda, M., and Sugiura, A. (1979). Origin of small RNA in von Magnus particles of influenza virus. J Virol 29, 1142-1148.

Nayak, D.P., Sivasubramanian, N., Davis, A.R., Cortini, R., and Sung, J. (1982). Complete sequence analyses show that two defective interfering influenza viral RNAs contain a single internal deletion of a polymerase gene. Proc Natl Acad Sci U S A 79, 2216-2220.

Nayak, D.P., Tobita, K., Janda, J.M., Davis, A.R., and De, B.K. (1978). Homologous interference mediated by defective interfering influenza virus derived from a temperaturesensitive mutant of influenza virus. J Virol 28, 375-386. 
Ngunjiri, J.M., Buchek, G.M., Mohni, K.N., Sekellick, M.J., and Marcus, P.I. (2013). Influenza virus subpopulations: exchange of lethal H5N1 virus NS for H1N1 virus NS triggers de novo generation of defective-interfering particles and enhances interferon-inducing particle efficiency. J Interferon Cytokine Res 33, 99-107.

Ngunjiri, J.M., Lee, C.W., Ali, A., and Marcus, P.I. (2012). Influenza virus interferoninducing particle efficiency is reversed in avian and mammalian cells, and enhanced in cells co-infected with defective-interfering particles. J Interferon Cytokine Res 32, 280-285.

Paules, C., and Subbarao, K. (2017). Influenza. Lancet 390, 697-708.

Perez-Cidoncha, M., Killip, M.J., Oliveros, J.C., Asensio, V.J., Fernandez, Y., Bengoechea, J.A., Randall, R.E., and Ortin, J. (2014). An unbiased genetic screen reveals the polygenic nature of the influenza virus anti-interferon response. J Virol 88, 4632-4646.

Saira, K., Lin, X., DePasse, J.V., Halpin, R., Twaddle, A., Stockwell, T., Angus, B., CozziLepri, A., Delfino, M., Dugan, V., et al. (2013). Sequence analysis of in vivo defective interfering-like RNA of influenza A H1N1 pandemic virus. J Virol 87, 8064-8074.

Schoggins, J.W., Wilson, S.J., Panis, M., Murphy, M.Y., Jones, C.T., Bieniasz, P., and Rice, C.M. (2011). A diverse range of gene products are effectors of the type I interferon antiviral response. Nature 472, 481-485.

Scott, P.D., Meng, B., Marriott, A.C., Easton, A.J., and Dimmock, N.J. (2011a). Defective interfering influenza A virus protects in vivo against disease caused by a heterologous influenza B virus. J Gen Virol 92, 2122-2132.

Scott, P.D., Meng, B., Marriott, A.C., Easton, A.J., and Dimmock, N.J. (2011b). Defective interfering influenza virus confers only short-lived protection against influenza virus disease: evidence for a role for adaptive immunity in DI virus-mediated protection in vivo. Vaccine $29,6584-6591$.

Seitz, C., Isken, B., Heynisch, B., Rettkowski, M., Frensing, T., and Reichl, U. (2012). Trypsin promotes efficient influenza vaccine production in MDCK cells by interfering with the antiviral host response. Appl Microbiol Biotechnol 93, 601-611.

Te Velthuis, A.J., and Fodor, E. (2016). Influenza virus RNA polymerase: insights into the mechanisms of viral RNA synthesis. Nat Rev Microbiol 14, 479-493.

Von Magnus, P. (1954). Incomplete forms of influenza virus. Adv Virus Res 2, 59-79.

Wang, C., Forst, C.V., Chou, T.W., Geber, A., Wang, M., Hamou, W., Smith, M., Sebra, R., Zhang, B., Zhou, B., et al. (2020). Cell-to-Cell Variation in Defective Virus Expression and Effects on Host Responses during Influenza Virus Infection. mBio 11.

Winkler, M., Bertram, S., Gnirss, K., Nehlmeier, I., Gawanbacht, A., Kirchhoff, F., Ehrhardt, C., Ludwig, S., Kiene, M., Moldenhauer, A.S., et al. (2012). Influenza A virus does not encode a tetherin antagonist with Vpu-like activity and induces IFN-dependent tetherin expression in infected cells. PLoS One 7, e43337. 
634

635

636

637

638

639

640

641

642

643

644

645

646

647

648

649

650

651

652

653

654

655

656

657

658

659

660

661

662

Winkler, M., Rice, S.A., and Stamminger, T. (1994). UL69 of human cytomegalovirus, an open reading frame with homology to ICP27 of herpes simplex virus, encodes a transactivator of gene expression. J Virol 68, 3943-3954.

Zimmermann, P., Manz, B., Haller, O., Schwemmle, M., and Kochs, G. (2011). The viral nucleoprotein determines Mx sensitivity of influenza A viruses. J Virol 85, 8133-8140.

Zmora, P., Molau-Blazejewska, P., Bertram, S., Walendy-Gnirss, K., Nehlmeier, I., Hartleib, A., Moldenhauer, A.S., Konzok, S., Dehmel, S., Sewald, K., et al. (2017). Non-human primate orthologues of TMPRSS2 cleave and activate the influenza virus hemagglutinin. PLoS One 12, e0176597.

\section{FIGURE LEGENDS}

\section{Figure 1. Antiviral activity of DI RNAs inversely correlates with DI RNA length in the} presence of trypsin

(A) DI-244 inhibits genome replication in the mini-replicon assay. 293T cells were transfected with plasmids encoding the viral polymerase proteins, NP, a segment 8-based luciferase reporter (mini-replicon system) and either empty plasmid or plasmid for expression of DI-244 mRNA (10 and $300 \mathrm{ng}$ ) and vRNA. Removing the plasmid encoding PB2 from the transfection mix served as negative control. Cotransfection of all support plasmids and empty plasmid instead of DI-244 encoding plasmid served as positive control. The average of five independent experiments is shown, for which the positive control was set as $100 \%$. Error bars indicate standard error of the mean (SEM).

(B) The truncated open reading frame of DI-244 does not contribute to inhibition of genome replication in the mini-replicon assay. The experiment was carried out as described for panel A but the cells were cotransfected with a plasmid for expression of DI-244 mRNA and vRNA with or without the first three ATGs of the PB2 ORF being intact (DI-244, DI-244 mut ATG), a plasmid for expression of DI-244 mRNA (DI-244 ORF) or empty plasmid pCAGGS. The average of three independent experiments is shown, for which the positive control was set as $100 \%$. Error bars indicate SEM. 
663 (C) The inhibitory activity of segment 1-derived DI RNAs in the mini-replicon assays is

inversely correlated with DI RNA length. The experiment was carried out as described for panel A but 300 ng of plasmids harboring the indicated segment 1-derived DI RNAs were cotransfected. The DI RNAs tested were numbered as shown in table S1. The average of five independent experiments is shown, for which the positive control was set as $100 \%$. Error bars indicate SEM.

(D) The inhibitory activity of segment 2- and 3-derived DI RNAs in the mini-replicon assays is inversely correlated with DI RNA length. The experiment was conducted as described for panel A but 300 ng of plasmids harboring the indicated segment 2 and 3-derived DI-RNAs were cotransfected. The DI RNAs tested were numbered as shown in table S1. The average of three independent experiments is shown, for which the positive control was set as $100 \%$. Error bars indicate SEM.

(E) The inhibitory activity of DI RNAs in the mini-replicon assays is independent from the origin of the reporter segment. The experiment was carried out as described for panel A but the indicated reporter segments and segment 1, 2 and 3-derived DI RNAs were used. The results of a single representative experiment are shown and were confirmed in an independent experiment. Error bars indicate standard deviation (SD).

(F) Antiviral activity of segment 1-derived DIPs is inversely correlated with DI RNA lengths in the presence of trypsin. MDCK cells were coinfected with the indicated DIPs (MOI 1) and $\mathrm{A} / \mathrm{PR} / 8 / 34$ (MOI 0.001 ) in the presence of trypsin, washed, and cultured in medium with trypsin. DIP-negative supernatants served as controls. At $72 \mathrm{~h}$ post infection, viral titers in culture supernatants were determined by focus formation assay. The average of four independent experiments is shown; error bars indicate SEM. In panels A-D statistical significance of differences between values measured for cells cotransfected with support plasmids and either empty plasmid (+ control) or DI RNA encoding plasmid was determined using one-way ANOVA with Sidak's posttest (panel A) and with Dunnett's posttest (panel B- 
689 D). In panel F statistical significance of differences between values measured for cells with 690 virus and DIPs at reciprocal DIP dilution was determined using one-way ANOVA with

691 Dunnett's posttest. *, $\mathrm{p} \leq 0.05 ; * *, \mathrm{p} \leq 0.01 ; * * *, \mathrm{p} \leq 0.001$

692

\section{Figure 2. Induction of the IFN system is a major contributor to DIP antiviral activity}

694 (A) Trypsin inactivates IFN $\alpha$. A549 wt cells were exposed to recombinant IFN $\alpha$ (100 U/ml) 695 in the presence and absence of serially diluted trypsin (T). Undiluted trypsin (IFN $\alpha+T)$ was 696 added at a concentration of $0.5 \mu \mathrm{g} / \mathrm{ml}$. After $24 \mathrm{~h}$, cells were harvested, RNA isolated and MX1 expression analyzed by quantitative RT-PCR. MX1 transcripts levels were normalized

698

699

700

701

702

703

704

705

706

707

708

709

710

711

712

713

against $\beta$-actin transcript levels. The average of three independent experiments is shown. Error bars indicate SEM.

(B) Pre-exposure of target cells to DIPs boosts DIP antiviral activity independent of trypsin. Left panel, - Trypsin condition: MDCK cells were either coinfected with DI-244 (MOI 10) and A/WSN/33 (MOI 0.1) in the absence of trypsin or DI-244 was added to cells at $24 \mathrm{~h}$ before virus. Cells were washed $1 \mathrm{~h}$ after addition of virus and maintained in growth medium. At $72 \mathrm{~h}$ post infection, viral titers in culture supernatants were determined by focus formation assay. Right panel, + trypsin condition: The experiment was carried out as described for the left panel, but A/WSN/33-derived DIPs (MOI 1) and A/WSN/33 (MOI 0.001) were used and maintained in infection medium supplemented with trypsin. The average of three independent experiments is shown in both panels; error bars indicate SEM.

(C) STAT1 is required MX1 induction by IAV and DIP. A549 cells and A549 STAT1 ${ }^{-/-}$cells were exposed to IFN $\alpha(100 \mathrm{U} / \mathrm{ml}), \mathrm{A} / \mathrm{PR} / 8 / 34$ or DI-244 (all MOI 1, in the presence of trypsin) for $1 \mathrm{~h}$, washed, incubated for $24 \mathrm{~h}$ in the absence of trypsin and MXI mRNA expression quantified using qRT-PCR. The average of five independent experiments is shown. Error bars indicate SEM. 
714 (D) Anti-IAV activity of DI-244 is partially and anti-VSV activity of DIP is fully dependent on STAT1. Antiviral activity of DI-244 was analyzed as described for the left panel of figure 2B but A549 wt and A549 STAT1 $^{-/}$cells were used. At $96 \mathrm{~h}$ post infection, viral titers in culture supernatants were determined by focus formation assay. The average of six (A/WSN/33) and three independent experiments (VSV) is shown. Error bars indicate SEM.

(E) DI RNA length does not modulate DIP antiviral activity in the context of a functional IFN system. Antiviral activity of the indicated DIPs was analyzed as described for panel D adding DIPs $24 \mathrm{~h}$ before virus. The average of five independent experiments is shown. Error bars indicate SEM.

In panels B and D statistical significance of differences between values measured for cells inoculated with DIPs at $24 \mathrm{~h}$ before IAV infection and cells to which IAV and DIPs were added at the same time was determined using two-way ANOVA with Sidak's posttest. In panel E statistical significance of differences between values measured for cells with virus and DIPs at reciprocal DIP dilution was determined using one-way ANOVA with Dunnett's posttest. *, $\mathrm{p} \leq 0.05 ; * *, \mathrm{p} \leq 0.01 ; * * *, \mathrm{p} \leq 0.001$

\section{Figure 3. DI-244 robustly induces ISG but not IFN expression}

(A) DI-244 does not induce IFN expression as determined in a VSV-replicon-based bioassay. A549 and A549 STAT1 $^{-/-}$cells were exposed to IAV, VSV or DI-244 and supernatants collected, heat inactivated, acid treated and added onto A549 cells followed by infection with VSV. For calibration, A549 cells were incubated with recombinant IFNa, VSV infected and infection efficiency was quantified. The average of three independent experiments is shown. Error bars indicate SEM.

(B) DI-244 induces robust ISG but not IFN expression as determined by RNAseq. A549 cells (top panel) and A549 STAT1 $^{-/-}$cells (bottom panel) were incubated with IAV (A/PR/8/34), DI-244 at a MOI of 1 in the absence of trypsin and control supernatants and subjected to 
740 RNAseq analysis. Expression of selected ISGs is shown. The average of two independent

741 experiments (A549) and three experiments (A549 STAT1 ${ }^{-/-}$) is presented. Error bars indicate 742 SEM.

743 (C) DI-244 induces robust ISG but not IFN expression as determined by qRT-PCR analysis. 744 The A549 wt cells described in panel B were subjected to qRT-PCR analysis of ISG 745 expression. The average of three independent experiments is shown. Error bars indicate SEM. 
Figure 1

Arora et al., 2020

A)

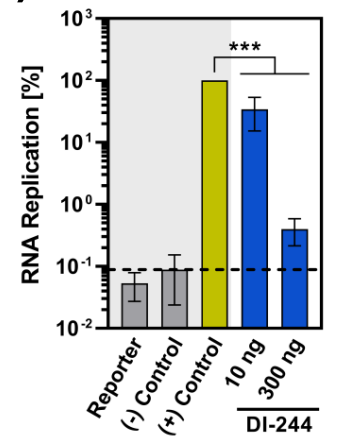

D)

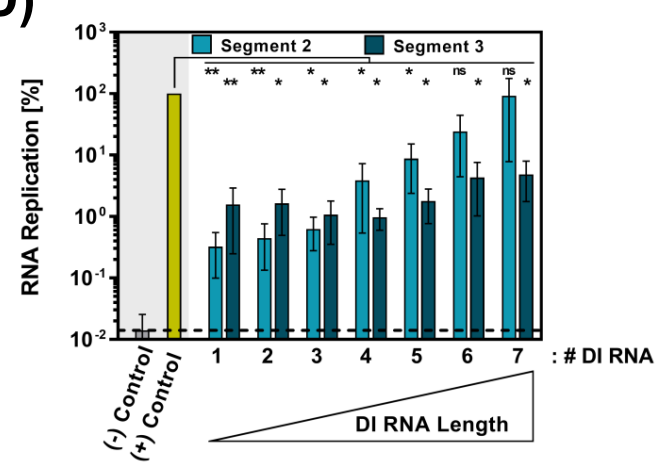

B)

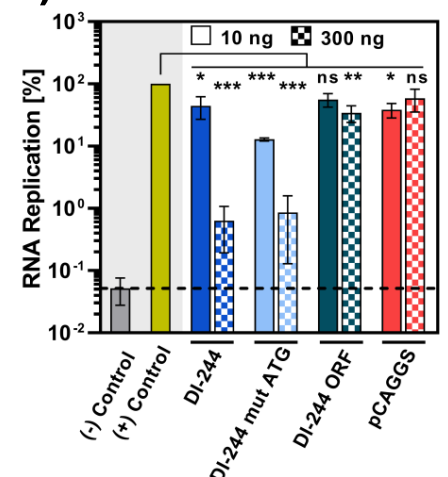

C)

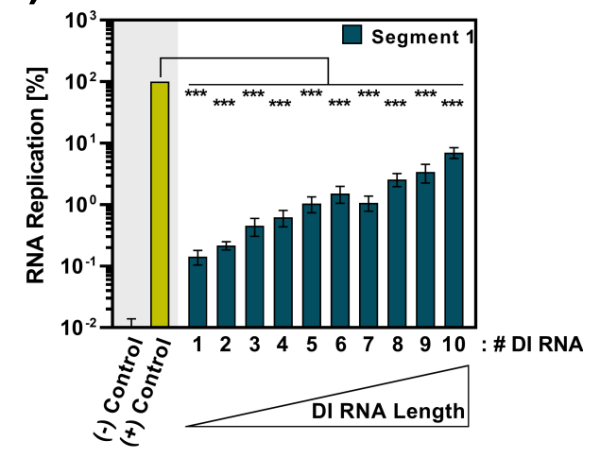

E)

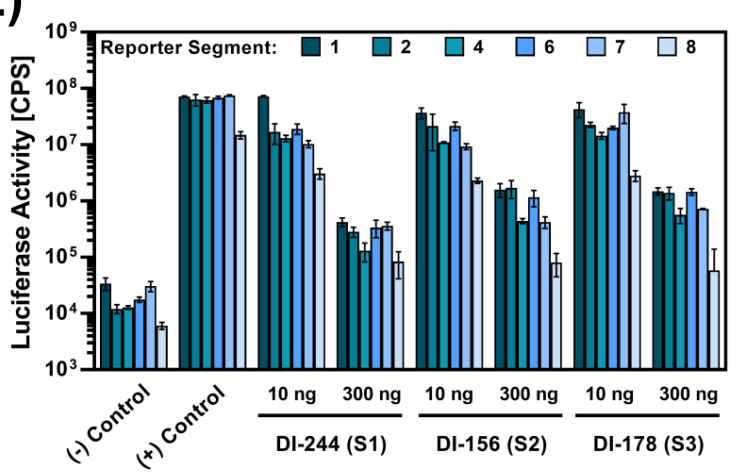

F)

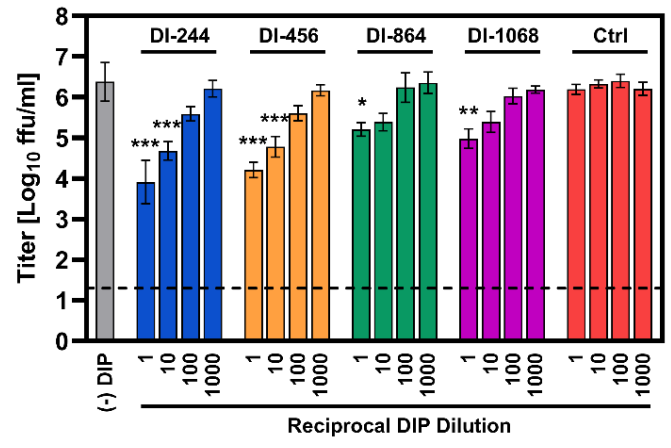


Figure 2

A)

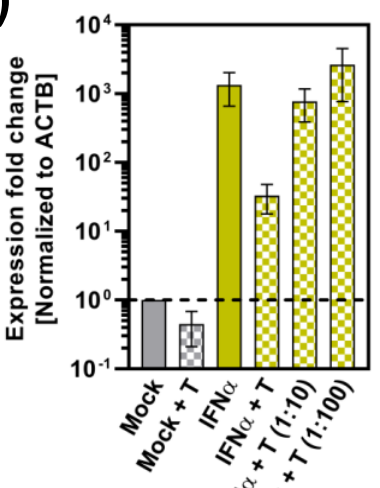

C)

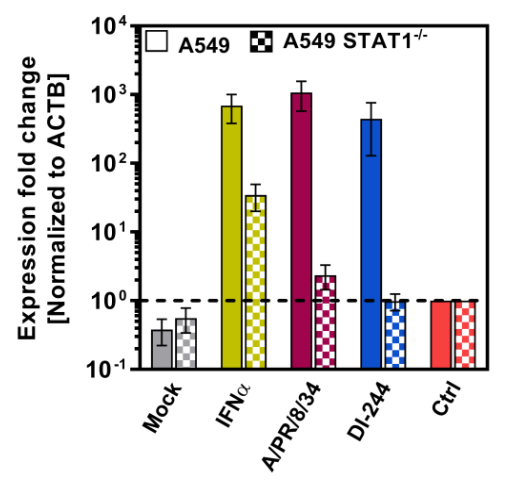

B)

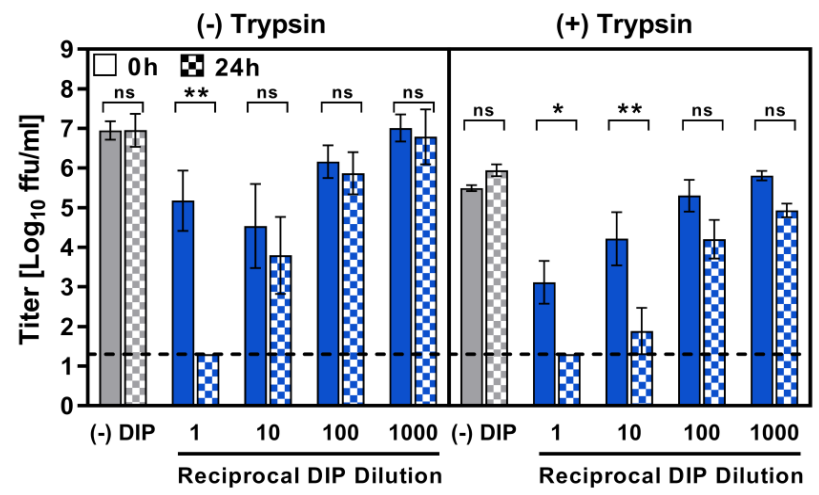

E)

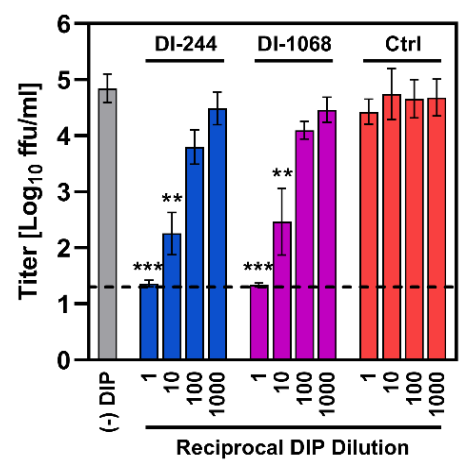

D)
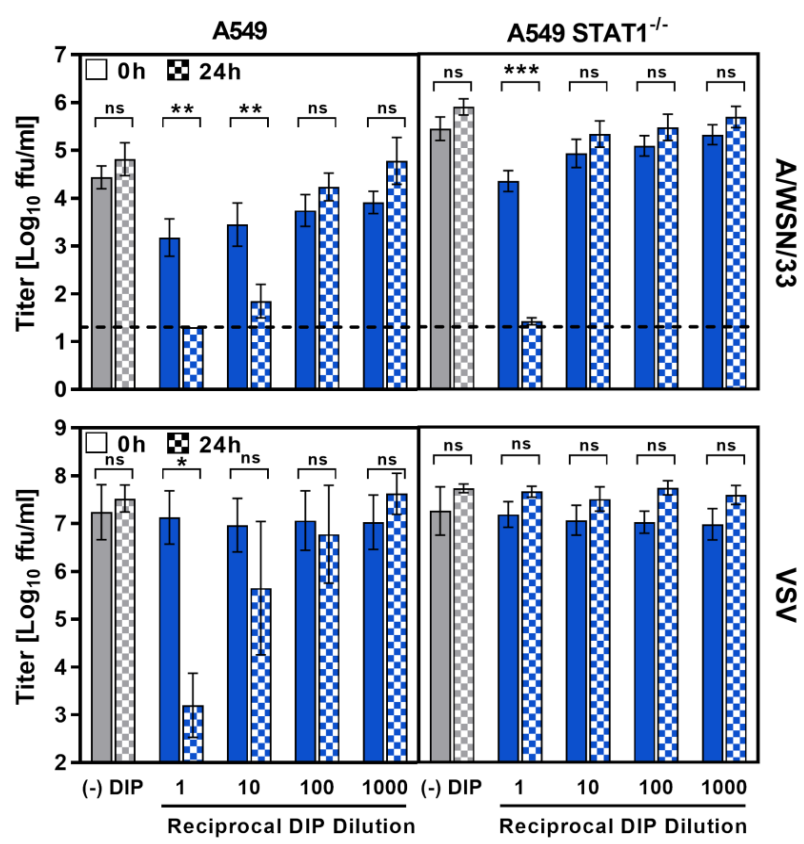
Figure 3

Arora et al., 2020

A)

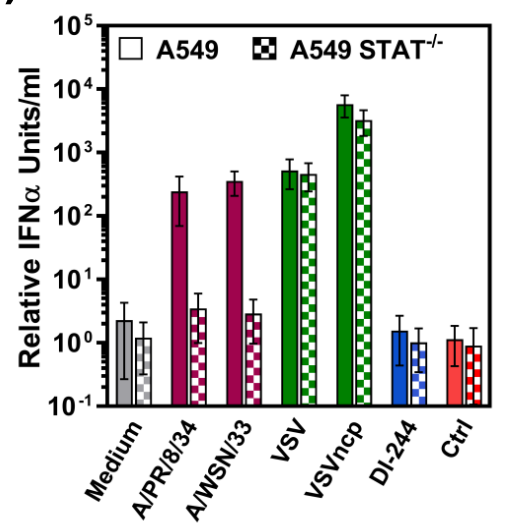

C)

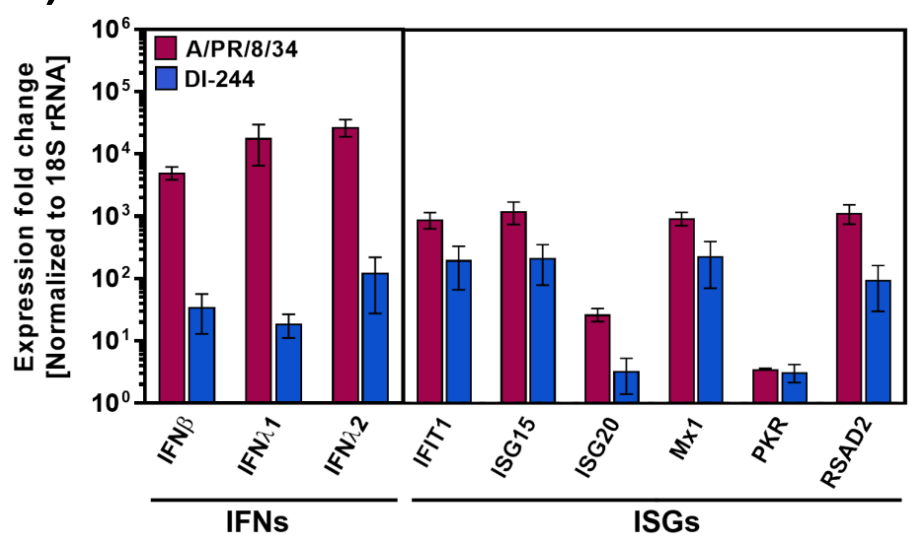

B)
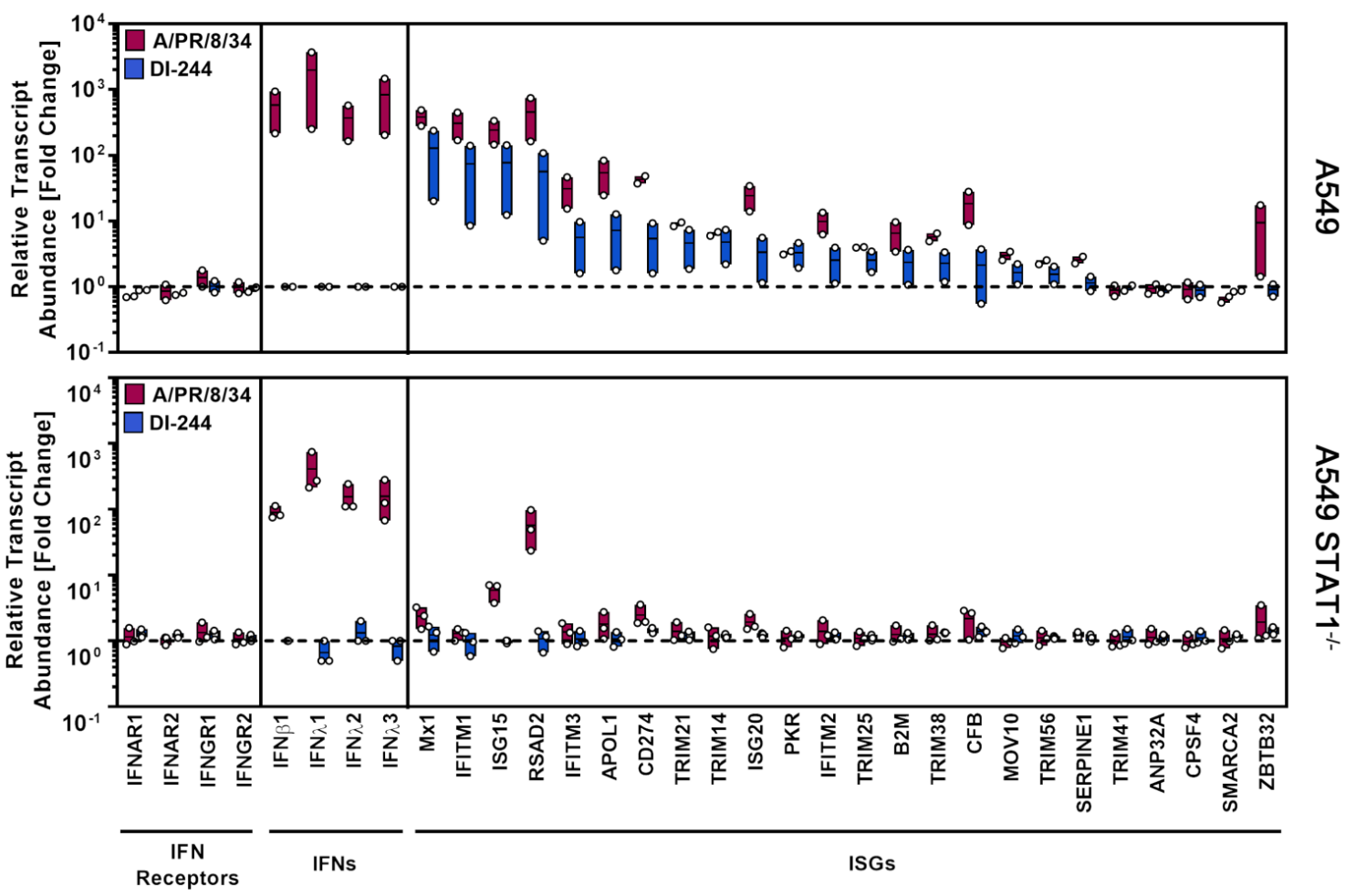


\begin{tabular}{|l|l|l|l|l|}
\hline \multicolumn{5}{|c|}{ Segment 1-derived DI-RNAs } \\
\hline Nr. & Name & Total (b) & 5' end (b) & 3' end (b) \\
\hline 1 & DI-244 & 421 & 151 & 244 \\
\hline 2 & DI-346 & 623 & 252 & 346 \\
\hline 3 & DI-448 & 829 & 354 & 448 \\
\hline 4 & DI-550 & 1032 & 456 & 550 \\
\hline 5 & DI-662 & 1236 & 558 & 652 \\
\hline 6 & DI-754 & 1440 & 660 & 754 \\
\hline 7 & DI-856 & 1645 & 762 & 856 \\
\hline 8 & DI-958 & 1849 & 864 & 958 \\
\hline 9 & DI-1060 & 2051 & 966 & 1060 \\
\hline 10 & DI-1162 & 2256 & 1068 & 1162 \\
\hline
\end{tabular}

Segment 2-derived DI-RNAs

\begin{tabular}{|l|l|l|l|l|}
\hline Nr. & Name & Total (b) & 5' end (b) & 3' end (b) \\
\hline 1 & DI-156 & 334 & 151 & 156 \\
\hline 2 & DI-258 & 537 & 252 & 258 \\
\hline 3 & DI-360 & 741 & 354 & 360 \\
\hline 4 & DI-462 & 945 & 456 & 462 \\
\hline 5 & DI-666 & 1353 & 660 & 666 \\
\hline 6 & DI-870 & 1761 & 864 & 870 \\
\hline 7 & DI-1074 & 2169 & 1068 & 1074 \\
\hline
\end{tabular}

Segment 3-derived DI-RNAs

\begin{tabular}{|l|l|l|l|l|}
\hline Nr. & Name & Total (b) & 5' end (b) & 3' end (b) \\
\hline 1 & DI-178 & 346 & 141 & 178 \\
\hline 2 & DI-280 & 550 & 243 & 280 \\
\hline 3 & DI-382 & 754 & 345 & 382 \\
\hline 4 & DI-484 & 958 & 447 & 484 \\
\hline 5 & DI-688 & 1366 & 651 & 688 \\
\hline 6 & DI-892 & 1774 & 855 & 892 \\
\hline 7 & DI-1096 & 2182 & 1059 & 1096 \\
\hline \multicolumn{7}{|c|}{ All constructs contain an mcs } \\
\hline \multicolumn{6}{|c|}{ located between the viral sequences } \\
\hline
\end{tabular}


Table S2

\begin{tabular}{|c|c|}
\hline \\
\hline \\
\hline \multicolumn{2}{|c|}{\begin{tabular}{l|l}
\multicolumn{2}{l}{ Oligonucleotides for cloning of DI-RNAs } \\
Name & Sequence \\
IAVseg1- & CAGGAAGACAGGAGA \\
DI244-for & \\
\end{tabular}} \\
\hline $\begin{array}{l}\text { IAVseg1- } \\
\text { DI244-rev }\end{array}$ & GAATGAGGAATCCCCTCAGTCTTCTCCTGTCTTCCTG \\
\hline $\begin{array}{l}\text { IAVseg1- } \\
\text { DI244rep-for }\end{array}$ & $\begin{array}{l}\text { TCAGGAAGACAGGAGAAGAGATCTGGTACCGCAGCGGCCGCTTA } \\
\text { ACTGAGGGGATTCCTCATT }\end{array}$ \\
\hline $\begin{array}{l}\text { IAVseg1- } \\
\text { DI244rep-rev }\end{array}$ & $\begin{array}{l}\text { AATGAGGAATCCCCTCAGTTAAGCGGCCGCTGCGGTACCAGATCT } \\
\text { CTTCTCCTGTCTTCCTGA }\end{array}$ \\
\hline DIP-346-for & $\begin{array}{l}\text { GAGAAATGAGCAAGGACAAGGATCCGGTACCGCAGCGGCCGCTT } \\
\text { AACTATAACAAGGCCACG }\end{array}$ \\
\hline DIP-346-rev & $\begin{array}{l}\text { CGTGGCCTTGTTATAGTTAAGCGGCCGCTGCGGTACCGGATCCTT } \\
\text { GTCCTTGCTCATTTCTC }\end{array}$ \\
\hline DIP-448-for & $\begin{array}{l}\text { CCAATAACAAATACAGTTGGATCCGGTACCGCAGCGGCCGCTTAA } \\
\text { GCCGCTCCACCAAAGCAA }\end{array}$ \\
\hline DIP-448-rev & $\begin{array}{l}\text { TTGCTTTGGTGGAGCGGCTTAAGCGGCCGCTGCGGTACCGGATCC } \\
\text { AACTGTATTTGTTATTGG }\end{array}$ \\
\hline DIP-550-for & $\begin{array}{l}\text { CAAGTCAAAATACGTCGGGGATCCGGTACCGCAGCGGCCGCTTA } \\
\text { AGAGGCCAATACAGTGGG }\end{array}$ \\
\hline DIP-550-rev & $\begin{array}{l}\text { CCCACTGTATTGGCCTCTTAAGCGGCCGCTGCGGTACCGGATCCC } \\
\text { CGACGTATTTTGACTTG }\end{array}$ \\
\hline DIP-652-for & $\begin{array}{l}\text { GTGGGAGCCAGGATACTAGGATCCGGTACCGCAGCGGCCGCTTA } \\
\text { AGAAACTGGGAAACTGTT }\end{array}$ \\
\hline DIP-652-rev & $\begin{array}{l}\text { AACAGTTTCCCAGTTTCTTAAGCGGCCGCTGCGGTACCGGATCCT } \\
\text { AGTATCCTGGCTCCCAC }\end{array}$ \\
\hline DIP-754-for & $\begin{array}{l}\text { CATGTTGGAGAGAGAACTGGGATCCGGTACCGCAGCGGCCGCTT } \\
\text { AACACAGGGAACAGAGAAAC }\end{array}$ \\
\hline DIP-754-rev & $\begin{array}{l}\text { GTTTCTCTGTTCCCTGTGTTAAGCGGCCGCTGCGGTACCGGATCCC } \\
\text { AGTTCTCTCTCCAACATG }\end{array}$ \\
\hline DIP-856-for & $\begin{array}{l}\text { CTGGGAACAGATGTATACTGGATCCGGTACCGCAGCGGCCGCTTA } \\
\text { AGAGTACTCCAGCACGGAGA }\end{array}$ \\
\hline DIP-856-rev & $\begin{array}{l}\text { TCTCCGTGCTGGAGTACTCTTAAGCGGCCGCTGCGGTACCGGATC } \\
\text { CAGTATACATCTGTTCCCAG }\end{array}$ \\
\hline DIP-958-for & $\begin{array}{l}\text { AGCAGATCCACTAGCATCTGGATCCGGTACCGCAGCGGCCGCTTA } \\
\text { ACCTATCGACAATGTGATGG }\end{array}$ \\
\hline DIP-958-rev & $\begin{array}{l}\text { CCATCACATTGTCGATAGGTTAAGCGGCCGCTGCGGTACCGGATC } \\
\text { CAGATGCTAGTGGATCTGCT }\end{array}$ \\
\hline DIP-1060-for & $\begin{array}{l}\text { CGTGGATATATGCAAGGCTGGATCCGGTACCGCAGCGGCCGCTTA } \\
\text { ATTTCGTCAATAGGGCGA }\end{array}$ \\
\hline DIP-1060-rev & $\begin{array}{l}\text { TCGCCCTATTGACGAAATTAAGCGGCCGCTGCGGTACCGGATCCA } \\
\text { GCCTTGCATATATCCACG }\end{array}$ \\
\hline DIP-1162-for & $\begin{array}{l}\text { GGAAGAGGTGCTTACGGGCGGATCCGGTACCGCAGCGGCCGCTT } \\
\text { AATAGTGAGTGGGAGAGACG }\end{array}$ \\
\hline DIP-1162-rev & $\begin{array}{l}\text { CGTCTCTCCCACTCACTATTAAGCGGCCGCTGCGGTACCGGATCC } \\
\text { GCCCGTAAGCACCTCTTCC }\end{array}$ \\
\hline DIP164-for & ATCAGGAAGACAGGAGAAGAGAAAGGAGAGAAGGCTAATG \\
\hline
\end{tabular}




\begin{tabular}{|c|c|}
\hline DIP164-rev & CATTAGCCTTCTCTCCTTTCTCTTCTCCTGTCTTCCTGAT \\
\hline DIP164P-for & AGACAGGAGAAGAACCCAGCGAAAGGAGAGAAGGCTAATG \\
\hline DIP164P-rev & CATTAGCCTTCTCTCCTTTCGCTGGGTTCTTCTCCTGTCT \\
\hline DIP164-80-for & GGATAACGGAAATGATTCCTGAAAGGAGAGAAGGCTAATG \\
\hline $\begin{array}{l}\text { DIP164-80- } \\
\text { rev }\end{array}$ & CATTAGCCTTCTCTCCTTTCAGGAATCATTTCCGTTATCC \\
\hline DIP204-for & ATCAGGAAGACAGGAGAAGAATGGGCCAGCACTAAGCATC \\
\hline DIP204-rev & GATGCTTAGTGCTGGCCCATTCTTCTCCTGTCTTCCTGAT \\
\hline $\begin{array}{l}\text { fluA AarI- } \\
\text { PB2-1G }\end{array}$ & CGATCACCTGCTCGAGGGAGCGAAAGCAGGTC \\
\hline $\begin{array}{l}\text { fluA AarI- } \\
\text { PB2-2341R }\end{array}$ & CGATCACCTGCTCTCTATTAGTAGAAACAAGGTCGTTT \\
\hline DIPS2-P-for & GGATACACCATGGATACTGAGCCCGAATTGATGCAC \\
\hline DIPS2-P-rev & GTGCATCAATTCGGGCTCAGTATCCATGGTGTATCC \\
\hline $\begin{array}{l}\text { DIPS2-P-mcs- } \\
\text { for }\end{array}$ & $\begin{array}{l}\text { GGATACACCATGGATACTGGTACCGCAGCGGCCGCTTAAGAGCC } \\
\text { CGAATTGATGCAC }\end{array}$ \\
\hline $\begin{array}{l}\text { DIPS2-P-mcs- } \\
\text { rev }\end{array}$ & $\begin{array}{l}\text { GTGCATCAATTCGGGCTCTTAAGCGGCCGCTGCGGTACCAGTATC } \\
\text { CATGGTGTATCC }\end{array}$ \\
\hline $\begin{array}{l}\text { DIPS2- } \\
252 \mathrm{mcs}-\text { for }\end{array}$ & $\begin{array}{l}\text { GGGCCACTGCCAGAAGACGGTACCGCAGCGGCCGCTTAAAAATG } \\
\text { TACCAAAGGTGCT }\end{array}$ \\
\hline $\begin{array}{l}\text { DIPS2- } \\
\text { 252mcs-rev }\end{array}$ & $\begin{array}{l}\text { AGCACCTTTGGTACATTTTTAAGCGGCCGCTGCGGTACCGTCTTCT } \\
\text { GGCAGTGGCCC }\end{array}$ \\
\hline $\begin{array}{l}\text { DIPS2- } \\
354 \mathrm{mcs}-\text { for }\end{array}$ & $\begin{array}{l}\text { AACTCGTGTATTGAAACGGGTACCGCAGCGGCCGCTTAAAAAAC } \\
\text { ATGGAGTATGATG }\end{array}$ \\
\hline $\begin{array}{l}\text { DIPS2- } \\
354 m c s-r e v\end{array}$ & $\begin{array}{l}\text { CATCATACTCCATGTTTTTTAAGCGGCCGCTGCGGTACCCGTTTCA } \\
\text { ATACACGAGTT }\end{array}$ \\
\hline $\begin{array}{l}\text { DIPS2- } \\
456 \mathrm{mcs}-\text { for }\end{array}$ & $\begin{array}{l}\text { GCTGCAACAGCATTGGCCGGTACCGCAGCGGCCGCTTAAATTACC } \\
\text { AGGGGCGTTTAT }\end{array}$ \\
\hline $\begin{array}{l}\text { DIPS2- } \\
456 \mathrm{mcs}-\mathrm{rev}\end{array}$ & $\begin{array}{l}\text { ATAAACGCCCCTGGTAATTTAAGCGGCCGCTGCGGTACCGGCCAA } \\
\text { TGCTGTTGCAGC }\end{array}$ \\
\hline $\begin{array}{l}\text { DIPS2- } \\
660 \mathrm{mcs}-\text { for }\end{array}$ & $\begin{array}{l}\text { AAAAAGAAGCAGAGATTGGGTACCGCAGCGGCCGCTTAATCATC } \\
\text { AAAGATTACAGGT }\end{array}$ \\
\hline $\begin{array}{l}\text { DIPS2- } \\
660 \mathrm{mcs}-\mathrm{rev}\end{array}$ & $\begin{array}{l}\text { ACCTGTAATCTTTGATGATTAAGCGGCCGCTGCGGTACCCAATCT } \\
\text { CTGCTTCTTTTT }\end{array}$ \\
\hline $\begin{array}{l}\text { DIPS2- } \\
864 m c s-f o r\end{array}$ & $\begin{array}{l}\text { GGCAATGAGAAGAAAGCAGGTACCGCAGCGGCCGCTTAAACATA } \\
\text { AACAGAACAGGTA }\end{array}$ \\
\hline $\begin{array}{l}\text { DIPS2- } \\
864 \text { mcs-rev }\end{array}$ & $\begin{array}{l}\text { TACCTGTTCTGTTTATGTTTAAGCGGCCGCTGCGGTACCTGCTTTC } \\
\text { TTCTCATTGCC }\end{array}$ \\
\hline $\begin{array}{l}\text { DIPS2- } \\
1068 \mathrm{mcs}-\text { for }\end{array}$ & $\begin{array}{l}\text { ATGTTCTCAAACAAAATGGGTACCGCAGCGGCCGCTTAATAAGCA } \\
\text { CTGTATTAGGCG }\end{array}$ \\
\hline $\begin{array}{l}\text { DIPS2- } \\
1068 \text { mcs-rev }\end{array}$ & $\begin{array}{l}\text { CGCCTAATACAGTGCTTATTAAGCGGCCGCTGCGGTACCCATTTT } \\
\text { GTTTGAGAACAT }\end{array}$ \\
\hline $\begin{array}{l}\text { fluA AarI- } \\
\text { PB1-1-4G }\end{array}$ & CGATCACCTGCTCGAGGGAGCGAAAGCAGGCA \\
\hline $\begin{array}{l}\text { fluA AarI- } \\
\text { PB1-2341R }\end{array}$ & CGATCACCTGCTCTCTATTAGTAGAAACAAGGCATTT \\
\hline DIPS3-P-for & AAATTTGCAGCAATATGCCCTGGGACCTTTGATCTT \\
\hline DIPS3-P-rev & AAGATCAAAGGTCCCAGGGCATATTGCTGCAAATTT \\
\hline DIPS3-P-mcs- & AAATTTGCAGCAATATGCAGATCTGGTACCGCAGCGGCCGCTTAA \\
\hline
\end{tabular}




\begin{tabular}{|c|c|}
\hline for & CCTGGGACCTTTGATCTT \\
\hline $\begin{array}{l}\text { DIPS3-P-mcs- } \\
\text { rev }\end{array}$ & $\begin{array}{l}\text { AAGATCAAAGGTCCCAGGTTAAGCGGCCGCTGCGGTACCAGATC } \\
\text { TGCATATTGCTGCAAATTT }\end{array}$ \\
\hline $\begin{array}{l}\text { DIPS3- } \\
243 \text { mcs-for }\end{array}$ & $\begin{array}{l}\text { CCAAATGCACTTTTGAAGAGATCTGGTACCGCAGCGGCCGCTTAA } \\
\text { TCGGTATTCAACAGCTTG }\end{array}$ \\
\hline $\begin{array}{l}\text { DIPS3- } \\
243 \text { mcs-rev }\end{array}$ & $\begin{array}{l}\text { CAAGCTGTTGAATACCGATTAAGCGGCCGCTGCGGTACCAGATCT } \\
\text { CTTCAAAAGTGCATTTGG }\end{array}$ \\
\hline $\begin{array}{l}\text { DIPS3- } \\
\text { 345mcs-for }\end{array}$ & $\begin{array}{l}\text { AAACCAAAGTTTCTACCAAGATCTGGTACCGCAGCGGCCGCTTAA } \\
\text { GAGTTCTTTGAGAACAAA }\end{array}$ \\
\hline $\begin{array}{l}\text { DIPS3- } \\
\text { 345mcs-rev }\end{array}$ & $\begin{array}{l}\text { TTTGTTCTCAAAGAACTCTTAAGCGGCCGCTGCGGTACCAGATCT } \\
\text { TGGTAGAAACTTTGGTTT }\end{array}$ \\
\hline $\begin{array}{l}\text { DIPS3- } \\
447 \mathrm{mcs}-\text { for }\end{array}$ & $\begin{array}{l}\text { AATAAAATTAAATCTGAGAGATCTGGTACCGCAGCGGCCGCTTAA } \\
\text { AAATGGGGAATGGAGATG }\end{array}$ \\
\hline $\begin{array}{l}\text { DIPS3- } \\
447 \mathrm{mcs}-\mathrm{rev}\end{array}$ & $\begin{array}{l}\text { CATCTCCATTCCCCATTTTTAAGCGGCCGCTGCGGTACCAGATCTC } \\
\text { TCAGATTTAATTTTATT }\end{array}$ \\
\hline $\begin{array}{l}\text { DIPS3- } \\
855 \text { rev-for }\end{array}$ & $\begin{array}{l}\text { AATGGGCCTCCCTGTTCTAGATCTGGTACCGCAGCGGCCGCTTAA } \\
\text { AGCATGAGAAGGAATTAT }\end{array}$ \\
\hline $\begin{array}{l}\text { DIPS3- } \\
855 \mathrm{mcs}-\mathrm{rev}\end{array}$ & $\begin{array}{l}\text { ATAATTCCTTCTCATGCTTTAAGCGGCCGCTGCGGTACCAGATCT } \\
\text { AGAACAGGGAGGCCCATT }\end{array}$ \\
\hline $\begin{array}{l}\text { DIPS3- } \\
651 \mathrm{mcs}-\mathrm{for}\end{array}$ & $\begin{array}{l}\text { AGGTTTGAAATCACAGGAAGATCTGGTACCGCAGCGGCCGCTTA } \\
\text { AAGATCCCACTTAAGGAAT }\end{array}$ \\
\hline $\begin{array}{l}\text { DIPS3- } \\
651 \mathrm{mcs}-\mathrm{rev}\end{array}$ & $\begin{array}{l}\text { ATTCCTTAAGTGGGATCTTTAAGCGGCCGCTGCGGTACCAGATCT } \\
\text { TCCTGTGATTTCAAACCT }\end{array}$ \\
\hline $\begin{array}{l}\text { DIPS3- } \\
\text { 1059mcs-for }\end{array}$ & $\begin{array}{l}\text { CAAGTACTGGCAGAACTGAGATCTGGTACCGCAGCGGCCGCTTA } \\
\text { AGAGAACATGGCACCAGAA }\end{array}$ \\
\hline $\begin{array}{l}\text { DIPS3- } \\
1059 \text { mcs-rev }\end{array}$ & $\begin{array}{l}\text { TTCTGGTGCCATGTTCTCTTAAGCGGCCGCTGCGGTACCAGATCTC } \\
\text { AGTTCTGCCAGTACTTG }\end{array}$ \\
\hline $\begin{array}{l}\text { fluA AarI- } \\
\text { PA1-1-4G }\end{array}$ & CGATCACCTGCTCGAGGGAGCGAAAGCAGGTAC \\
\hline $\begin{array}{l}\text { fluA AarI- } \\
\text { PA1-2233R }\end{array}$ & CGATCACCTGCTCTCTATTAGTAGAAACAAGGTACTT \\
\hline
\end{tabular}


Table S3

Oligonucleotides used for cloning of replicon reporter constructs

\begin{tabular}{|c|c|}
\hline Name & Sequence \\
\hline $\begin{array}{l}\text { fluA AarI- } \\
\text { NS-1 }\end{array}$ & CGATCACCTGCTCGAGGGAGCAAAAGCAGGGTG \\
\hline $\begin{array}{l}\text { fluA AarI- } \\
\text { NS-890R }\end{array}$ & CGATCACCTGCTCTCTATTAGTAGAAACAAGGGTGTTTT \\
\hline $\begin{array}{l}\text { fluA AarI- } \\
\text { seg2rep-5 }\end{array}$ & $\begin{array}{l}\text { CGATCACCTGCTCGAGGGAGCGAAAGCAGGCAAACCATTTGAATG } \\
\text { GAAGACGCCAAAAACATAAAG }\end{array}$ \\
\hline $\begin{array}{l}\text { fluA AarI- } \\
\text { seg2rep-3 }\end{array}$ & $\begin{array}{l}\text { CGATCACCTGCTCTCTATTAGTAGAAACAAGGCATTTTTTCATGAAG } \\
\text { GACAAGCTAAATTCATTACACGGCGATCTTTCCG }\end{array}$ \\
\hline $\begin{array}{l}\text { fluA AarI- } \\
\text { seg4rep-5 }\end{array}$ & $\begin{array}{l}\text { CGATCACCTGCTCGAGGGAGCAAAAGCAGGGGAAAATAAAAACAA } \\
\text { CCAAAATGGAAGACGCCAAAAACATAAAG }\end{array}$ \\
\hline $\begin{array}{l}\text { fluA AarI- } \\
\text { seg4rep-3 }\end{array}$ & $\begin{array}{l}\text { CGATCACCTGCTCTCTATTAGTAGAAACAAGGGTGTTTTTCCTCATA } \\
\text { TCTCTGAAATTCTAATCTTACACGGCGATCTTTCCG }\end{array}$ \\
\hline $\begin{array}{l}\text { fluA AarI- } \\
\text { seg6rep-5 }\end{array}$ & $\begin{array}{l}\text { CGATCACCTGCTCGAGGGAGCAAAAGCAGGAGTTTAAAATGGAAG } \\
\text { ACGCCAAAAACATAAAG }\end{array}$ \\
\hline $\begin{array}{l}\text { fluA AarI- } \\
\text { seg6rep-3 }\end{array}$ & $\begin{array}{l}\text { CGATCACCTGCTCTCTATTAGTAGAAACAAGGAGTTTTTTGAACAG } \\
\text { ATTACACGGCGATCTTTCCG }\end{array}$ \\
\hline $\begin{array}{l}\text { fluA AarI- } \\
\text { seg7rep-5 }\end{array}$ & $\begin{array}{l}\text { CGATCACCTGCTCGAGGGAGCAAAAGCAGGTAGATATTGAAAGAT } \\
\text { GGAAGACGCCAAAAACATAAAG }\end{array}$ \\
\hline $\begin{array}{l}\text { fluA AarI- } \\
\text { seg7rep-3 }\end{array}$ & $\begin{array}{l}\text { CGATCACCTGCTCTCTATTAGTAGAAACAAGGTAGTTTTTTACACGG } \\
\text { CGATCTTTCCG }\end{array}$ \\
\hline $\begin{array}{l}\text { fluA AarI- } \\
\text { repPB2-5 }\end{array}$ & $\begin{array}{l}\text { CGATCACCTGCTCGAGGGAGCGAAAGCAGGTCAATTATATTCAATA } \\
\text { TGGAAGACGCCAAAAACATAAAG }\end{array}$ \\
\hline $\begin{array}{l}\text { fluA AarI- } \\
\text { repPB2-3 }\end{array}$ & $\begin{array}{l}\text { CGATCACCTGCTCTCTATTAGTAGAAACAAGGTCGTTTTTAAACTAT } \\
\text { TCGACATTACACGGCGATCTTTCCG }\end{array}$ \\
\hline
\end{tabular}




\section{Discussion}

\subsection{First Manuscript - A system for production of defective interfering particles in the absence of infectious influenza $A$ virus}

DIPs appear naturally during influenza virus infections at high $\mathrm{MOI}$ and were first observed in embryonated chicken eggs while performing serial passaging of influenza A virus (Magnus 1954). Subsequently, it was discovered that DIPs can protect cell cultures and animals from wt influenza virus infection, indicating that they could be developed as antiviral agents (Dimmock et al. 2008; Easton et al. 2011). However, their production was dependent on the presence of wt virus which needed to be UV-inactivated. To tackle this limitation, a cell linebased system for production of DIPs in the absence of wt virus was established. This lessened safety concerns and resulted in substantial homogeneity of DIP preparation and thus could facilitate DIP use in humans.

To produce DI-244 particles in the absence of wt virus, 293T and MDCK cell lines stably expressing the IAV polymerase subunit PB2 were generated using retroviral technology. This approach was chosen since the PB2 ORF was inactivated in DI-244 (which was derived from IAV genomic segment 1) and providing the PB2 protein in trans should allow amplification of DIPs harbouring wt genomic segments 2-8 jointly with DI-244. 293T cells were chosen for DIP production because of high transfectability while MDCK cells were used because of high permissiveness to IAV infection. The DI-244 production in these 293T/MDCK cocultures yielded about $10^{6} \mathrm{DI}-244$ infectious particles $/ \mathrm{ml}$ when quantified using MDCK PB2opt cells for focus formation assay - the first time that DIP infectivity has been ever quantified. A similar study also generated about $10^{7}$ DI-244 PFU/ml using AX4 cells stably expressing PB2 protein, but they mutated all start codons of DI-244 RNA to prevent unexpected expression of unrelated proteins (Yamagata et al. 2019). Previous studies reported about 10 to 100-fold higher yields of DI-244 in embryonated chicken eggs, amplified in cell-culture, and bioreactors but these production systems depended on use of wt virus and reported viral genome copies/ml and not infectious unit/ml (Dimmock et al. 2008; Frensing et al. 2013; Wasik et al. 2018). Considering that only a fraction of particles containing RNA will also be infectious and interfering, the particle yields obtained here can be considered robust. Moreover, this study was the first to report DIP production in the absence of wt IAV and thus did not encompass UV irradiation to inactivate wt IAV. UV inactivation relies on the fact that DI-244 RNA is much smaller than the corresponding wt RNA and thus much less likely to be 
inactivated by UV. Moreover, any inactivating mutation in other segments will abrogate infectivity of wt IAV but not DIPs, since the latter only need to deliver their DI-RNA into wt IAV coinfected cells to ensure their amplification. Considering that UV inactivation can be inefficient for large scale production of DIPs and yields variable results, the newly established DIP production system constitutes a significant advance.

The 293T and MDCK cells used for DI-244 production were equipped with expression cassettes for codon optimized PB2 to ensure efficient expression and to avoid potential recombination events with $\mathrm{DI}-244$, which would result in the production of wt IAV. The use of codon optimized PB2 increased PB2 expression in MDCK cells and improved DIP yields about 100-fold. Additionally, no evidence of recombination events between DI-244 RNA and PB2 RNA was neither observed with confocal microscopy or immunofluorescence, nor quantified through focus formation assay or RT-PCR analysis. For visual examination of DI244 production and spread, DI-244-mScarlet was generated as it encodes a red fluorescent protein. The robust production of DI-244-mScarlet in PB2opt expressing cells raised the question of whether the particles exerted antiviral activity. MDCK cells co-infected with PR8 and DI-244-mScarlet demonstrated that DI-244 displayed robust and concentrationdependent antiviral activity. Moreover, antiviral activity was also observed with a H3N2 IAV, A/Panama/2007/99, but not with VSV. The inhibition of diverse IAV by DI-244 was expected from published studies (Dimmock et al. 2008) and reflected replication interference, i.e. the DI-RNA outcompetes wt RNAs for viral and cellular genome replication requirements (Dimmock and Easton 2014, 2015). The lack of VSV inhibition is noteworthy since DIPs are known to inhibit heterologous viruses (Easton et al. 2011; Scott et al. 2011a) by eliciting an IFN response (Scott et al. 2011a, 2011b) and not by genome competition (Scott et al. 2011c; Easton et al. 2011). The IFN response was likely suppressed in the cell culture systems used in the present study. This is the effect of trypsin that was used to ensure robust IAV and DIP spread but inactivates type I IFN (Seitz et al. 2012). This means that antiviral activity incurred by DIPS is largely due to replication interference in the case of IAV but not VSV which lacks genome competition. Robust antiviral activity of DIPs was observed when these particles were used at a 100 or 1000 -fold excess as compared to wt IAV. This DIP/wt virus ratio is similar to that used for previously published animal studies (IAV/DIP ratio of 1:3400 (determined by qRT-PCR analysis (Dimmock and Easton 2015). 
What are the implications of the above discussed findings for DIP use in humans? A DIP to virus ratio of 1000:1 was required for robust DIP antiviral activity in cell culture. It could be speculated that a lower ratio will suffice in humans, as DIPs can stimulate IFN responses in vivo although those responses were probably not stimulated in the cell culture systems used to determine DIP antiviral activity, as discussed below. Considering that DIPs reside for a long time in the respiratory tract of mice, and studies with DIP-treated animals reported one week protection after treatment (Dimmock et al. 2008; Dimmock and Easton 2015), DIP stability in the respiratory tract of humans should not be considered as an impediment to provide protection against influenza virus, which needs to be further investigated. Finally, it could be stated that although DIPs can reassort with wt IAV in coinfected cells, such a reassortment is unlikely to raise safety concerns since, first, the largely apathogenic PR8 was used for DIP production, and second, recombination of wt IAV with PR8 would not increase virulence of reassortant virus as compared to the wt virus.

The results discussed above were obtained with DI-244-mScarlet particles. Pure DI-244 wt particles were expected to be produced under same conditions. Unexpectedly, this was not the case and a potential explanation for this issue was provided by results obtained in the mini-replicon assay (discussed in second manuscript). DI-244 RNA suppressed replication of IAV segments, and the inhibitory activity was found to be length dependent. This indicated that DI-244 wt might have higher antiviral activity than DI-244-mScarlet and that expression of high amounts of DI-244 wt might auto-inhibit segment amplification and particle production. Further analysis of the results showed that the efficiency of particle production of DI-244 wt in PB2opt cells inversely correlated with the amount of DI-244 wt plasmid transfected.

In summary, this study demonstrated that cell lines expressing PB2opt allow production of DI-244 particles in the absence of wt virus. The particles exerted anti-IAV activity and their infectivity could be determined by focus formation assay. No recombination events between DI-244 RNA and PB2 full-length RNA were observed by immunofluorescence microscopy, focus forming assay and RT-PCR analysis, indicating that DIPs produced in the system are homogenous and safe. Thus, a DIP production system not relying on wt virus was reported for the first time, which shows the feasibility to develop DIPs for antiviral therapies. 


\subsection{Second Manuscript - Interferon induction and not replication interference is the major determinant of anti-influenza virus activity of defective interfering particles}

DI-RNAs are found in IAV infected eggs (Magnus 1954), cell cultures (Dimmock et al. 2008; Frensing et al. 2013), animals (Bean et al. 1985; Barrett and Dimmock 1986; Chambers and Webster 1987; Dimmock and Easton 2014, 2015), and patients (Saira et al. 2013; Vasilijevic et al. 2017). They interfere with the replication of the wt IAVs from which they originated but also inhibit unrelated viruses (Easton et al. 2011; Scott et al. 2011a). Replication interference and IFN induction are important mechanisms by which DIPs inhibit IAV (Vignuzzi and López 2019). DIPs suppress influenza virus infection by interfering with genome replication (a process termed replication interference) and by stimulating immune response (IFN induction) (Dimmock and Easton 2014, 2015; Scott et al. 2011a, 2011b). However, the relative contribution of replication interference and IFN induction to DIP antiviral as well as the mechanism underlying IFN induction are incompletely understood. The present study provides evidence that the contribution of replication interference to DIP antiviral activity is minor as compared to IFN induction. Moreover, the study reveals that DIP-dependent activation of the IFN system encompasses induction of ISG but not IFN expression.

The concept behind replication interference posits that DI-RNA outcompete their wt counterparts for viral and cellular resources required for replication because they are smaller and thus replicate faster (Li and Pattnaik 1997; Calain and Roux 1995). On the other hand, the concept has not been systematically investigated. To close this gap, variants of genomic segments 1, 2, and 3 with nested deletions were generated. A mini-replicon assay, which measures genome replication and mRNA expression, showed that DI-RNA length inversely correlated with inhibitory activity. Besides that, any deletion in segment 1, 2 and 3 was sufficient to convert these RNAs into DI-RNAs and inhibitory activity was independent of the target segment, in keeping with findings reported by Meng and colleagues (Meng et al. 2017). These findings support the replication interference concept and suggest that any deletion of sequences located between the conserved regions at the $5^{\prime}$ and $3^{\prime}$ ends of genomic segments, which are required for translation and transcription, should generate DIRNAs. Indeed, the $5^{\prime}$ and $3^{\prime}$ ends of DI-RNAs were reported exhibit similar sequences that are conserved in all influenza virus strains (Saira et al. 2013; Jennings et al. 1983).

It has been reported that DI-RNAs are frequently generated from genomic IAV segments 1-3, which are the largest segments of the viral genome and encode polymerase subunits (Davis 
and Nayak 1979). The reason for the preferential accumulation of deletions in these segments is their length, which makes them prone to internal deletions during genome replication (Nayak et al. 1985). Although the deletions present in these DI-RNAs are central to antiviral activity, the expression of truncated viral proteins from these DI-RNAs could contribute to antiviral activity (Boergeling et al. 2015). However, the truncated PB2 protein encoded by $\mathrm{DI}-244$ did not contribute to the antiviral activity. Thus, overexpression of the truncated PB2 protein did not result in inhibition of IAV genome replication and mutating ATG start codons in DI-244 did not affect the inhibitory activity of DI-244 (Meng et al. 2017).

To investigate a potential contribution of IFN to DIP antiviral activity, it was necessary to set up conditions to ensure activation of hemagglutinin (HA) protein without trypsin as it inactivates IFN (Seitz et al. 2012). For this, IAV strain A/WSN/33 (WSN) was used, which does not depend on HA activation by trypsin for acquisition of infectivity. Addition of WSNderived DIPs to target cells at $24 \mathrm{~h}$ before addition of IAV (subsequently termed $24 \mathrm{~h}$ setting) boosted antiviral activity as compared to addition of DIPs and IAV at the same time (subsequently termed $0 \mathrm{~h}$ setting). This suggested that activation of the IFN system could play a major role in DIP antiviral activity. Unexpectedly, the presence or absence of trypsin did not modulate antiviral activity, indicating that the enhanced antiviral activity observed in the $24 \mathrm{~h}$ setting did not require production of IFN- $\alpha$. Finally, it is worth mentioning that DIRNA segment length did not impact antiviral activity in the $24 \mathrm{~h}$ setting, suggesting that different mechanisms account for DIP antiviral activity in the 0 and $24 \mathrm{~h}$ setting

In order to determine the contribution of IFN to DIP antiviral activity, IFN-competent human A549 wt cells and A549 STAT1 ${ }^{-/}$cells were used. Type I IFN triggers the expression of about 400 genes, many of which encode proteins with antiviral activity, including $M x 1$ (Schoggins et al. 2011). To confirm the STAT1 knock-out on a functional level, $M \times 1$-induction by IAV and DI-244 particles were analysed. Both particles induced $M \times 1$ expression in A549 wt cells but not in A549 STAT1 ${ }^{-/}$cells. Holzinger and colleagues reported similar findings for IAV but in their study $M \times 1$ induction by IAV was less efficient as compared to IFN- $\alpha$ (Holzinger et al. 2007). In contrast, Marcos-Villar and colleagues and the present study observed the opposite trend (Marcos-Villar et al. 2018). Substantial differences in the IFN- $\alpha$ preparations, concentrations, conditions used in the presence and absence of trypsin might account for 
differences in the findings. Nevertheless, the results are in concordance with the expected defect in IFN signalling in A549 STAT1 ${ }^{-/-}$cells.

It was then investigated whether DI-244 antiviral activity was STAT1-dependent, using the 24 h setting. When A549 STAT1 ${ }^{-/-}$cells were exposed to the highest amount of DIPs analysed, potent anti-IAV activity was observed. Nonetheless, when 10-fold diluted DI-244 was analysed, markedly reduced anti-IAV activity was observed in STAT1 ${ }^{-/}$cells as compared to wt cells. Thus, the anti-IAV activity of DI-244 was partially dependent on an intact STAT1 gene. In contrast, the ability of DI-244 to inhibit VSV infection was fully STAT1-dependent, independent of the DIP dilution analysed. Several observations could explain why IAV inhibition by DI-244 was not STAT1-dependent when the highest amount of DIPS was investigated. First, it is possible that IAV inhibition under those conditions was independent of the IFN system. Apparently, cells exposed to a high amount of DI-RNAs can induce a prosurvival program, dependent on RLR signalling pathway, which may protect cells from dying during infection (Vignuzzi and López 2019). Second, it is possible that certain ISGs with antiIAV activity are upregulated by DI-244 in a STAT1-independent fashion. This agrees with a previously study which reported that STAT1 gene is dispensable for IRF3 dependent stimulation of ISG expression (Wang et al. 2017). Third, another possibility is that IRF9 and STAT2 could induce an antiviral effect independent of STAT1 by fusion of IRF9 with transcriptional activation domain of STAT2 containing binding sites for transcription coregulators (Kraus et al. 2003). Finally, unphosphorylated-ISGF3 alone can induce an antiviral effect (Cheon et al. 2013). Collectively, it can be stated, DI-244 induces anti-IAV activity partially independent of STAT1.

The finding that DI-244 anti-IAV activity was partially STAT1-dependent whereas anti-VSVactivity was fully dependent open two possibilities. Whether DI-244 induces an antiviral state through IFN expression, which signals in a STAT1-dependent manner, or directly through ISG expression, which is the consequence of IFN induced, STAT1-dependent signalling. To determine whether DI-244 induced expression of type I, II, III IFNs in A549 cells a bioassay was performed. Briefly, supernatants harvested from IAV, VSV or DI-244 exposed cells were heated and acid treated (followed by neutralization) to inactivate residual virus. As it has been reported that both type I (IFN- $\beta$ ) and type III (IFN- $\lambda$ ) are acid-stable, but only type I (IFN- $\beta$ ) displays resistance to alkaline treatment (Berger Rentsch and Zimmer 2011). 
However, it needs to be stated that another study reported that IFN- $\lambda$ is acid-sensitive (Reid et al. 2016). Afterwards, supernatants or IFN- $\alpha$ (used for standardization) was inoculated onto fresh A549 cells in order to trigger an antiviral state, followed by VSV infection and quantification of infection. The quantification showed that supernatant of IAV-infected cells induced an antiviral state in target cells that inhibited VSV infection in a STAT1-dependent manner, as expected. Similar results were obtained for cells exposed to supernatants from VSV infected cells, but inhibition was independent of STAT1. Surprisingly, supernatants from DI-244 exposed cells did not induce any antiviral state.

To confirm that DIPs do not induce IFN and to determine whether DIPs induce expression of ISGs, RNAseq analysis was conducted. This analysis showed that neither IAV nor DI-244 induced expression of IFN receptors while IAV but not DI-244 induced expression of IFN. Regardless of the discrepancy in upregulation of IFNs by IAV and DI-244, both induced expression of a broad panel of ISGs, although IAV was more efficient than DI-244 in ISG induction. These results were confirmed with qRT-PCR and concluded that DI-244 induces ISGs in a STAT1-dependent but IFN-independent manner. Notably, a recent study confirmed that DIPs can efficiently induce ISGs in the absence of robust type I and III IFN expression (Wang et al. 2020). The mechanism behind this induction is unknown. In the absence of IFN, expression of a subset of ISGs can be induced by IRF7 (Schmid et al. 2010). For instance, ISG56 is upregulated by IRF3 in an IFN-independent manner through ISREs present in the ISG56 promoter (Grandvaux et al. 2002). However, in the present screen, no STAT1independent DIP induced upregulation of ISGs with anti-IAV activity was observed.

In conclusion, this study provides evidence that induction of IFN system is a major contributor of DIP antiviral activity. Having said this, the induction of IFN system does not involve DIP-mediated expression of IFN but direct induction of ISG expression. 


\section{Publication bibliography}

Bailey, Charles C.; Huang, I-Chueh; Kam, Christina; Farzan, Michael (2012): Ifitm3 Limits the Severity of Acute Influenza in Mice. In PLoS pathogens 8 (9). DOI: 10.1371/journal.ppat.1002909.

Balachandran, Siddharth; Roberts, Paul C.; Brown, Laura E.; Truong, Ha; Pattnaik, Asit K.; Archer, David R.; Barber, Glen N. (2000): Essential Role for the dsRNA-Dependent Protein Kinase PKR in Innate Immunity to Viral Infection. In Immunity 13 (1), pp. 129-141. DOI: 10.1016/S1074-7613(00)00014-5.

Barrett, A. D.; Crouch, C. F.; Dimmock, N. J. (1984): Defective interfering Semliki Forest virus populations are biologically and physically heterogeneous. In The Journal of general virology 65 (Pt 8), pp. 1273-1283. DOI: 10.1099/0022-1317-65-8-1273.

Barrett, A. D.; Dimmock, N. J. (1986): Defective interfering viruses and infections of animals. In Current topics in microbiology and immunology 128, pp. 55-84. DOI: 10.1007/978-3-64271272-2_2.

Baum, Alina; Sachidanandam, Ravi; García-Sastre, Adolfo (2010): Preference of RIG-I for short viral RNA molecules in infected cells revealed by next-generation sequencing. In Proceedings of the National Academy of Sciences of the United States of America 107 (37), pp. 16303-16308. DOI: 10.1073/pnas.1005077107.

Bean, W. J.; Kawaoka, Y.; Wood, J. M.; Pearson, J. E.; Webster, R. G. (1985): Characterization of virulent and avirulent A/chicken/Pennsylvania/83 influenza $A$ viruses: potential role of defective interfering RNAs in nature. In Journal of virology 54 (1), pp. 151-160.

Bennett, John E.; Dolin, Raphael; Blaser, Martin J.; Mandell, Gerald L. Principles and practice of infectious diseases; Douglas, R. Gordon (2015): Mandell, Douglas, and Bennett's principles and practice of infectious diseases. Eighth edition. Philadelphia, PA: Elsevier/Saunders.

Berger Rentsch, Marianne; Zimmer, Gert (2011): A vesicular stomatitis virus replicon-based bioassay for the rapid and sensitive determination of multi-species type I interferon. In PloS one 6 (10), e25858. DOI: 10.1371/journal.pone.0025858.

Blanc, Mathieu; Hsieh, Wei Yuan; Robertson, Kevin A.; Kropp, Kai A.; Forster, Thorsten; Shui, Guanghou et al. (2013): The Transcription Factor STAT-1 Couples Macrophage Synthesis of 25-Hydroxycholesterol to the Interferon Antiviral Response. In Immunity 38 (1), pp. 106-118. DOI: 10.1016/j.immuni.2012.11.004.

Boergeling, Yvonne; Rozhdestvensky, Timofey S.; Schmolke, Mirco; Resa-Infante, Patricia; Robeck, Thomas; Randau, Gerrit et al. (2015): Evidence for a Novel Mechanism of Influenza Virus-Induced Type I Interferon Expression by a Defective RNA-Encoded Protein. In PLoS pathogens 11 (5), e1004924. DOI: 10.1371/journal.ppat.1004924.

Böttcher, Eva; Matrosovich, Tatyana; Beyerle, Michaela; Klenk, Hans-Dieter; Garten, Wolfgang; Matrosovich, Mikhail (2006): Proteolytic activation of influenza viruses by serine proteases TMPRSS2 and HAT from human airway epithelium. In Journal of virology 80 (19), pp. 9896-9898. DOI: 10.1128/JVI.01118-06. 
Bouvier, Nicole M.; Palese, Peter (2008): The biology of influenza viruses. In Vaccine 26 Suppl 4, D49-53. DOI: 10.1016/j.vaccine.2008.07.039.

Brass, Abraham L.; Huang, I-Chueh; Benita, Yair; John, Sinu P.; Krishnan, Manoj N.; Feeley, Eric M. et al. (2009): The IFITM proteins mediate cellular resistance to influenza A H1N1 virus, West Nile virus, and dengue virus. In Cell 139 (7), pp. 1243-1254. DOI: 10.1016/j.cell.2009.12.017.

Breen, Michael; Nogales, Aitor; Baker, Steven F.; Martínez-Sobrido, Luis (2016): ReplicationCompetent Influenza A Viruses Expressing Reporter Genes. In Viruses 8 (7). DOI: 10.3390/v8070179.

Cai, Xin; Chiu, Yu-Hsin; Chen, Zhijian J. (2014): The cGAS-cGAMP-STING pathway of cytosolic DNA sensing and signaling. In Molecular cell 54 (2), pp. 289-296. DOI: 10.1016/j.molcel.2014.03.040.

Calain, P.; Roux, L. (1995): Functional characterisation of the genomic and antigenomic promoters of Sendai virus. In Virology 212 (1), pp. 163-173. DOI: 10.1006/viro.1995.1464.

CDC (2019a): 1918 Pandemic. (H1N1 virus) (Past Pandemics). Available online at https://www.cdc.gov/flu/pandemic-resources/1918-pandemic-h1n1.html, updated on $3 / 20 / 2019$, checked on 2/7/2020.

CDC (2019b): 1957-1958 Pandemic. (H2N2 virus) (Past Pandemics). Available online at https://www.cdc.gov/flu/pandemic-resources/1957-1958-pandemic.html, updated on $1 / 2 / 2019$, checked on $2 / 7 / 2020$.

CDC (2019c): 1968 Pandemic. (H3N2 virus) (Past Pandemics). Available online at https://www.cdc.gov/flu/pandemic-resources/1968-pandemic.html, updated on 1/2/2019, checked on $2 / 7 / 2020$.

CDC (2019d): 2009 H1N1 Pandemic (Summary of Progress since 2009). Available online at https://www.cdc.gov/flu/pandemic-resources/h1n1-summary.htm, updated on 6/6/2019, checked on $2 / 7 / 2020$.

Chaipan, Chawaree; Kobasa, Darwyn; Bertram, Stephanie; Glowacka, Ilona; Steffen, Imke; Tsegaye, Theodros Solomon et al. (2009): Proteolytic activation of the 1918 influenza virus hemagglutinin. In Journal of virology 83 (7), pp. 3200-3211. DOI: 10.1128/JVI.02205-08.

Chambers, T. M.; Webster, R. G. (1987): Defective interfering virus associated with A/Chicken/Pennsylvania/83 influenza virus. In Journal of virology 61 (5), pp. 1517-1523.

Cheon, HyeonJoo; Holvey-Bates, Elise G.; Schoggins, John W.; Forster, Samuel; Hertzog, Paul; Imanaka, Naoko et al. (2013): IFN $\beta$-dependent increases in STAT1, STAT2, and IRF9 mediate resistance to viruses and DNA damage. In The EMBO Journal 32 (20), pp. 2751-2763. DOI: 10.1038/emboj.2013.203.

Coccia, Eliana M.; Severa, Martina; Giacomini, Elena; Monneron, Danièle; Remoli, Maria Elena; Julkunen, Ilkka et al. (2004): Viral infection and Toll-like receptor agonists induce a differential expression of type 1 and lambda interferons in human plasmacytoid and monocyte-derived dendritic cells. In European journal of immunology 34 (3), pp. 796-805. DOI: 10.1002/eji.200324610. 
Coelingh, Kathleen L.; Luke, Catherine J.; Jin, Hong; Talaat, Kawsar R. (2014): Development of live attenuated influenza vaccines against pandemic influenza strains. In Expert review of vaccines 13 (7), pp. 855-871. DOI: 10.1586/14760584.2014.922417.

Cox, N. J.; Subbarao, K. (2000): Global epidemiology of influenza: past and present. In Annual review of medicine 51, pp. 407-421. DOI: 10.1146/annurev.med.51.1.407.

Cromer, Deborah; van Hoek, Albert Jan; Jit, Mark; Edmunds, W. John; Fleming, Douglas; Miller, Elizabeth (2014): The burden of influenza in England by age and clinical risk group: a statistical analysis to inform vaccine policy. In The Journal of infection 68 (4), pp. 363-371. DOI: 10.1016/j.jinf.2013.11.013.

Darnell, J. E.; Kerr, I. M.; Stark, G. R. (1994): Jak-STAT pathways and transcriptional activation in response to IFNs and other extracellular signaling proteins. In Science (New York, N. Y.) 264 (5164), pp. 1415-1421. DOI: 10.1126/science.8197455.

Davis, A. R.; Nayak, D. P. (1979): Sequence relationships among defective interfering influenza viral RNAs. In Proceedings of the National Academy of Sciences of the United States of America 76 (7), pp. 3092-3096. DOI: 10.1073/pnas.76.7.3092.

Dawood, Fatimah S.; Jain, Seema; Finelli, Lyn; Shaw, Michael W.; Lindstrom, Stephen; Garten, Rebecca J. et al. (2009): Emergence of a novel swine-origin influenza A (H1N1) virus in humans. In The New England journal of medicine 360 (25), pp. 2605-2615. DOI: 10.1056/NEJMoa0903810.

De, B. K.; Nayak, D. P. (1980): Defective interfering influenza viruses and host cells: establishment and maintenance of persistent influenza virus infection in MDBK and HeLa cells. In Journal of virology 36 (3), pp. 847-859.

Di Pietro, Andrea; Kajaste-Rudnitski, Anna; Oteiza, Alexandra; Nicora, Lucia; Towers, Greg J.; Mechti, Nadir; Vicenzi, Elisa (2013): TRIM22 inhibits influenza A virus infection by targeting the viral nucleoprotein for degradation. In Journal of virology 87 (8), pp. 4523-4533. DOI: 10.1128/JVI.02548-12.

Dimmock, Nigel J.; Dove, Brian K.; Meng, Bo; Scott, Paul D.; Taylor, Irene; Cheung, Linda et al. (2012a): Comparison of the protection of ferrets against pandemic 2009 influenza A virus (H1N1) by 244 DI influenza virus and oseltamivir. In Antiviral research 96 (3), pp. 376-385. DOI: 10.1016/j.antiviral.2012.09.017.

Dimmock, Nigel J.; Dove, Brian K.; Scott, Paul D.; Meng, Bo; Taylor, Irene; Cheung, Linda et al. (2012b): Cloned defective interfering influenza virus protects ferrets from pandemic 2009 influenza A virus and allows protective immunity to be established. In PloS one 7 (12), e49394. DOI: 10.1371/journal.pone.0049394.

Dimmock, Nigel J.; Easton, Andrew J. (2014): Defective interfering influenza virus RNAs: time to reevaluate their clinical potential as broad-spectrum antivirals? In Journal of virology 88 (10), pp. 5217-5227. DOI: 10.1128/JVI.03193-13.

Dimmock, Nigel J.; Easton, Andrew J. (2015): Cloned Defective Interfering Influenza RNA and a Possible Pan-Specific Treatment of Respiratory Virus Diseases. In Viruses 7 (7), pp. 37683788. DOI: 10.3390/v7072796. 
Dimmock, Nigel J.; Rainsford, Edward W.; Scott, Paul D.; Marriott, Anthony C. (2008): Influenza virus protecting RNA: an effective prophylactic and therapeutic antiviral. In Journal of virology 82 (17), pp. 8570-8578. DOI: 10.1128/JVI.00743-08.

Dittmann, Meike; Hoffmann, Hans-Heinrich; Scull, Margaret A.; Gilmore, Rachel H.; Bell, Kierstin L.; Ciancanelli, Michael et al. (2015): A serpin shapes the extracellular environment to prevent influenza A virus maturation. In Cell 160 (4), pp.631-643. DOI: 10.1016/j.cell.2015.01.040.

Domingues, Patricia; Hale, Benjamin G. (2017): Functional Insights into ANP32A-Dependent Influenza A Virus Polymerase Host Restriction. In Cell Reports 20 (11), pp. 2538-2546. DOI: 10.1016/j.celrep.2017.08.061.

Dornfeld, Dominik; Dudek, Alexandra H.; Vausselin, Thibaut; Günther, Sira C.; Hultquist, Judd F.; Giese, Sebastian et al. (2018): SMARCA2-regulated host cell factors are required for MxA restriction of influenza A viruses. In Scientific reports 8 (1), p. 2092. DOI: 10.1038/s41598018-20458-2.

Dou, Dan; Revol, Rebecca; Östbye, Henrik; Wang, Hao; Daniels, Robert (2018): Influenza A Virus Cell Entry, Replication, Virion Assembly and Movement. In Frontiers in Immunology 9. DOI: 10.3389/fimmu.2018.01581.

Dubois, Julia; Traversier, Aurélien; Julien, Thomas; Padey, Blandine; Lina, Bruno; Bourdon, Jean-Christophe et al. (2019): The Nonstructural NS1 Protein of Influenza Viruses Modulates TP53 Splicing through Host Factor CPSF4. In Journal of virology 93 (7). DOI: 10.1128/JVI.02168-18.

Duhaut, S. D.; Dimmock, N. J. (1998): Heterologous protection of mice from a lethal human H1N1 influenza A virus infection by H3N8 equine defective interfering virus: comparison of defective RNA sequences isolated from the DI inoculum and mouse lung. In Virology 248 (2), pp. 241-253. DOI: 10.1006/viro.1998.9267.

Duhaut, S. D.; Dimmock, N. J. (2002): Defective segment 1 RNAs that interfere with production of infectious influenza $A$ virus require at least 150 nucleotides of 5 ' sequence: evidence from a plasmid-driven system. In The Journal of general virology 83 (Pt 2), pp. 403411. DOI: 10.1099/0022-1317-83-2-403.

Durfee, Larissa A.; Lyon, Nancy; Seo, Kyungwoon; Huibregtse, Jon M. (2010): The ISG15 conjugation system broadly targets newly synthesized proteins: implications for the antiviral function of ISG15. In Molecular cell 38 (5), pp. 722-732. DOI: 10.1016/j.molcel.2010.05.002.

Easton, Andrew J.; Scott, Paul D.; Edworthy, Nicole L.; Meng, Bo; Marriott, Anthony C.; Dimmock, Nigel J. (2011): A novel broad-spectrum treatment for respiratory virus infections: influenza-based defective interfering virus provides protection against pneumovirus infection in vivo. In Vaccine 29 (15), pp. 2777-2784. DOI: 10.1016/j.vaccine.2011.01.102.

Eckert, Nadine; Wrensch, Florian; Gärtner, Sabine; Palanisamy, Navaneethan; Goedecke, Ulrike; Jäger, Nils et al. (2014): Influenza A virus encoding secreted Gaussia luciferase as useful tool to analyze viral replication and its inhibition by antiviral compounds and cellular proteins. In PloS one 9 (5), e97695. DOI: 10.1371/journal.pone.0097695. 
Fensterl, Volker; Chattopadhyay, Saurabh; Sen, Ganes C. (2015): No Love Lost Between Viruses and Interferons. In Annual review of virology 2 (1), pp.549-572. DOI: 10.1146/annurev-virology-100114-055249.

Ferguson, Lucas; Eckard, Laura; Epperson, William B.; Long, Li-Ping; Smith, David; Huston, Carla et al. (2015): Influenza D virus infection in Mississippi beef cattle. In Virology 486, pp. 28-34. DOI: 10.1016/j.virol.2015.08.030.

Fink, Karin; Grandvaux, Nathalie (2013): STAT2 and IRF9: Beyond ISGF3. In JAK-STAT 2 (4), e27521. DOI: 10.4161/jkst.27521.

Frensing, Timo; Heldt, Frank Stefan; Pflugmacher, Antje; Behrendt, Ilona; Jordan, Ingo; Flockerzi, Dietrich et al. (2013): Continuous influenza virus production in cell culture shows a periodic accumulation of defective interfering particles. In PloS one 8 (9), e72288. DOI: 10.1371/journal.pone.0072288.

Frost, W. H. (1919): The Epidemiology of Influenza. In Public Health Reports (1896-1970) 34 (33), p. 1823. DOI: 10.2307/4575271.

Fu, X. Y.; Schindler, C.; Improta, T.; Aebersold, R.; Darnell, J. E. (1992): The proteins of ISGF-3, the interferon alpha-induced transcriptional activator, define a gene family involved in signal transduction. In Proceedings of the National Academy of Sciences of the United States of America 89 (16), pp. 7840-7843. DOI: 10.1073/pnas.89.16.7840.

Gallo-Ramírez, Lilí Esmeralda; Nikolay, Alexander; Genzel, Yvonne; Reichl, Udo (2015): Bioreactor concepts for cell culture-based viral vaccine production. In Expert review of vaccines 14 (9), pp. 1181-1195. DOI: 10.1586/14760584.2015.1067144.

Gao, Song; Malsburg, Alexander von der; Paeschke, Susann; Behlke, Joachim; Haller, Otto; Kochs, Georg; Daumke, Oliver (2010): Structural basis of oligomerization in the stalk region of dynamin-like MxA. In Nature 465 (7297), pp. 502-506. DOI: 10.1038/nature08972.

Gay, Nicholas J.; Symmons, Martyn F.; Gangloff, Monique; Bryant, Clare E. (2014): Assembly and localization of Toll-like receptor signalling complexes. In Nat Rev Immunol 14 (8), pp. 546-558. DOI: 10.1038/nri3713.

Ghebrehewet, Sam; MacPherson, Peter; Ho, Antonia (2016): Influenza. In BMJ (Clinical research ed.) 355, i6258. DOI: 10.1136/bmj.i6258.

Gil, M. P.; Bohn, E.; O'Guin, A. K.; Ramana, C. V.; Levine, B.; Stark, G. R. et al. (2001): Biologic consequences of Stat1-independent IFN signaling. In Proceedings of the National Academy of Sciences of the United States of America 98 (12), pp.6680-6685. DOI: 10.1073/pnas.111163898.

Goto, H.; Kawaoka, Y. (1998): A novel mechanism for the acquisition of virulence by a human influenza A virus. In Proceedings of the National Academy of Sciences of the United States of America 95 (17), pp. 10224-10228. DOI: 10.1073/pnas.95.17.10224.

Goto, H.; Wells, K.; Takada, A.; Kawaoka, Y. (2001): Plasminogen-binding activity of neuraminidase determines the pathogenicity of influenza A virus. In Journal of virology 75 (19), pp. 9297-9301. DOI: 10.1128/JVI.75.19.9297-9301.2001. 
Goubau, Delphine; Schlee, Martin; Deddouche, Safia; Pruijssers, Andrea J.; Zillinger, Thomas; Goldeck, Marion et al. (2014): Antiviral immunity via RIG-I-mediated recognition of RNA bearing 5'-diphosphates. In Nature 514 (7522), pp. 372-375. DOI: 10.1038/nature13590.

Goujon, Caroline; Moncorgé, Olivier; Bauby, Hélène; Doyle, Tomas; Ward, Christopher C.; Schaller, Torsten et al. (2013): Human MX2 is an interferon-induced post-entry inhibitor of HIV-1 infection. In Nature 502 (7472), pp. 559-562. DOI: 10.1038/nature12542.

Grandvaux, Nathalie; Servant, Marc J.; tenOever, Benjamin; Sen, Ganes C.; Balachandran, Siddarth; Barber, Glen N. et al. (2002): Transcriptional profiling of interferon regulatory factor 3 target genes: direct involvement in the regulation of interferon-stimulated genes. In Journal of virology 76 (11), pp. 5532-5539. DOI: 10.1128/jvi.76.11.5532-5539.2002.

Grohskopf, Lisa A.; Alyanak, Elif; Broder, Karen R.; Walter, Emmanuel B.; Fry, Alicia M.; Jernigan, Daniel B. (2019): Prevention and Control of Seasonal Influenza with Vaccines: Recommendations of the Advisory Committee on Immunization Practices - United States, 2019-20 Influenza Season. In MMWR. Recommendations and reports : Morbidity and mortality weekly report. Recommendations and reports 68 (3), pp.1-21. DOI: 10.15585/mmwr.rr6803a1.

Haller, Otto; Gao, Song; Malsburg, Alexander von der; Daumke, Oliver; Kochs, Georg (2010): Dynamin-like MxA GTPase: structural insights into oligomerization and implications for antiviral activity. In The Journal of biological chemistry 285 (37), pp. 28419-28424. DOI: 10.1074/jbc.R110.145839.

Haller, Otto; Staeheli, Peter; Schwemmle, Martin; Kochs, Georg (2015): Mx GTPases: dynamin-like antiviral machines of innate immunity. In Trends in microbiology 23 (3), pp. 154-163. DOI: 10.1016/j.tim.2014.12.003.

Han, Julianna; Perez, Jasmine; Schafer, Adam; Cheng, Han; Peet, Norton; Rong, Lijun; Manicassamy, Balaji (2018): Influenza Virus: Small Molecule Therapeutics and Mechanisms of Antiviral Resistance. In Current medicinal chemistry 25 (38), pp.5115-5127. DOI: 10.2174/0929867324666170920165926.

Hause, Ben M.; Collin, Emily A.; Liu, Runxia; Huang, Bing; Sheng, Zizhang; Lu, Wuxun et al. (2014): Characterization of a novel influenza virus in cattle and Swine: proposal for a new genus in the Orthomyxoviridae family. In mBio 5 (2), e00031-14. DOI: 10.1128/mBio.0003114.

Hay, A. J.; Wolstenholme, A. J.; Skehel, J. J.; Smith, M. H. (1985): The molecular basis of the specific anti-influenza action of amantadine. In The EMBO Journal 4 (11), pp. 3021-3024. DOI: 10.1002/j.1460-2075.1985.tb04038.x.

Hayashi, Tsuyoshi; MacDonald, Leslie A.; Takimoto, Toru (2015): Influenza A Virus Protein PA-X Contributes to Viral Growth and Suppression of the Host Antiviral and Immune Responses. In Journal of virology 89 (12), pp. 6442-6452. DOI: 10.1128/JVI.00319-15.

Hayden, F. G.; Cote, K. M.; Douglas, R. G. (1980): Plaque inhibition assay for drug susceptibility testing of influenza viruses. In Antimicrobial agents and chemotherapy 17 (5), pp. 865-870. DOI: 10.1128/aac.17.5.865. 
Hayden, Frederick G.; Sugaya, Norio; Hirotsu, Nobuo; Lee, Nelson; Jong, Menno D. de; Hurt, Aeron C. et al. (2018): Baloxavir Marboxil for Uncomplicated Influenza in Adults and Adolescents. In The New England journal of medicine 379 (10), pp.913-923. DOI: 10.1056/NEJMoa1716197.

Hegde, Nagendra R. (2015): Cell culture-based influenza vaccines: A necessary and indispensable investment for the future. In Human vaccines \& immunotherapeutics 11 (5), pp. 1223-1234. DOI: 10.1080/21645515.2015.1016666.

Hinson, Ella R.; Cresswell, Peter (2009a): The antiviral protein, viperin, localizes to lipid droplets via its $\mathrm{N}$-terminal amphipathic alpha-helix. In Proceedings of the National Academy of Sciences of the United States of America 106 (48), pp. 20452-20457. DOI: 10.1073/pnas.0911679106.

Hinson, Ella R.; Cresswell, Peter (2009b): The N-terminal amphipathic alpha-helix of viperin mediates localization to the cytosolic face of the endoplasmic reticulum and inhibits protein secretion. In The Journal of biological chemistry 284 (7), pp.4705-4712. DOI: 10.1074/jbc.M807261200.

Hirst, George K.; Pons, Marcel W. (1973): Mechanism of influenza recombination. In Virology 56 (2), pp. 620-631. DOI: 10.1016/0042-6822(73)90063-9.

Hoffmann, E. (2002): Eight-plasmid system for rapid generation of influenza virus vaccines. In Vaccine 20 (25-26), pp. 3165-3170. DOI: 10.1016/S0264-410X(02)00268-2.

Hoffmann, E.; Neumann, G.; Hobom, G.; Webster, R. G.; Kawaoka, Y. (2000a): "Ambisense" approach for the generation of influenza A virus: vRNA and mRNA synthesis from one template. In Virology 267 (2), pp. 310-317. DOI: 10.1006/viro.1999.0140.

Hoffmann, E.; Neumann, G.; Kawaoka, Y.; Hobom, G.; Webster, R. G. (2000b): A DNA transfection system for generation of influenza A virus from eight plasmids. In Proceedings of the National Academy of Sciences of the United States of America 97 (11), pp. 6108-6113. DOI: $10.1073 /$ pnas.100133697.

Holland, J. J.; Doyle, M. (1973): Attempts to Detect Homologous Autointerference In vivo with Influenza Virus and Vesicular Stomatitis Virus. In Infection and Immunity 7 (4), pp. 526531.

Holzinger, Dirk; Jorns, Carl; Stertz, Silke; Boisson-Dupuis, Stéphanie; Thimme, Robert; Weidmann, Manfred et al. (2007): Induction of MxA gene expression by influenza A virus requires type I or type III interferon signaling. In Journal of virology 81 (14), pp. 7776-7785. DOI: 10.1128/JVI.00546-06.

Honda, Kenya; Takaoka, Akinori; Taniguchi, Tadatsugu (2006): Type I interferon corrected gene induction by the interferon regulatory factor family of transcription factors. In Immunity 25 (3), pp. 349-360. DOI: 10.1016/j.immuni.2006.08.009.

Houser, Katherine; Subbarao, Kanta (2015): Influenza vaccines: challenges and solutions. In Cell host \& microbe 17 (3), pp. 295-300. DOI: 10.1016/j.chom.2015.02.012.

Huang, A. S.; Baltimore, D. (1970): Defective viral particles and viral disease processes. In Nature 226 (5243), pp. 325-327. DOI: 10.1038/226325a0. 
Hussain, Mazhar; Galvin, Henry D.; Haw, Tatt Y.; Nutsford, Ashley N.; Husain, Matloob (2017): Drug resistance in influenza A virus: the epidemiology and management. In Infection and drug resistance 10, pp. 121-134. DOI: 10.2147/IDR.S105473.

Isaacs, A.; Lindenmann, J. (1957): Virus interference. I. The interferon. By A. Isaacs and J. Lindenmann, 1957. In Journal of interferon research 7 (5), pp.429-438. DOI: 10.1089/jir.1987.7.429.

Isaacs, A.; Lindenmann, J.; VALENTINE, R. C. (1957): Virus interference. II. Some properties of interferon. In Proceedings of the Royal Society of London. Series B, Biological sciences 147 (927), pp. 268-273. DOI: 10.1098/rspb.1957.0049.

Ison, Michael G. (2015): Optimizing antiviral therapy for influenza: understanding the evidence. In Expert review of anti-infective therapy 13 (4), pp.417-425. DOI: 10.1586/14787210.2015.1018183.

Iwasaki, Akiko; Pillai, Padmini S. (2014): Innate immunity to influenza virus infection. In Nature reviews. Immunology 14 (5), pp. 315-328. DOI: 10.1038/nri3665.

Jaworski, Elizabeth; Routh, Andrew (2017): Parallel ClickSeq and Nanopore sequencing elucidates the rapid evolution of defective-interfering RNAs in Flock House virus. In PLoS pathogens 13 (5), e1006365. DOI: 10.1371/journal.ppat.1006365.

Jennings, Philip A.; Finch, John T.; Winter, Greg; Robertson, James S. (1983): Does the higher order structure of the influenza virus ribonucleoprotein guide sequence rearrangements in influenza viral RNA? In Cell 34 (2), pp. 619-627. DOI: 10.1016/0092-8674(83)90394-x.

Jia, Rui; Xu, Fengwen; Qian, Jin; Yao, Yunfang; Miao, Chunhui; Zheng, Yi-Min et al. (2014): Identification of an endocytic signal essential for the antiviral action of IFITM3. In Cellular microbiology 16 (7), pp. 1080-1093. DOI: 10.1111/cmi.12262.

Jin, Zhinan; Smith, Lucas K.; Rajwanshi, Vivek K.; Kim, Baek; Deval, Jerome (2013): The ambiguous base-pairing and high substrate efficiency of T-705 (Favipiravir) Ribofuranosyl 5'triphosphate towards influenza A virus polymerase. In PLOS ONE 8 (7), e68347. DOI: 10.1371/journal.pone.0068347.

Jordan, Ingo; Vos, Ad; Beilfuss, Stefanie; Neubert, Andreas; Breul, Sabine; Sandig, Volker (2009): An avian cell line designed for production of highly attenuated viruses. In Vaccine 27 (5), pp. 748-756. DOI: 10.1016/j.vaccine.2008.11.066.

Kato, Hiroki; Takeuchi, Osamu; Mikamo-Satoh, Eriko; Hirai, Reiko; Kawai, Tomoji; Matsushita, Kazufumi et al. (2008): Length-dependent recognition of double-stranded ribonucleic acids by retinoic acid-inducible gene-I and melanoma differentiation-associated gene 5. In The Journal of experimental medicine 205 (7), pp. 1601-1610. DOI: 10.1084/jem.20080091.

Klenk, Hans-Dieter; Rott, Rudolf; Orlich, Michaela; Blödorn, Jochen (1975): Activation of influenza A viruses by trypsin treatment. In Virology 68 (2), pp. 426-439. DOI: 10.1016/00426822(75)90284-6.

Kolakofsky, Daniel (1976): Isolation and characterization of Sendai virus DI-RNAs. In Cell 8 (4), pp. 547-555. DOI: 10.1016/0092-8674(76)90223-3. 
Kotenko, Sergei V.; Gallagher, Grant; Baurin, Vitaliy V.; Lewis-Antes, Anita; Shen, Meiling; Shah, Nital K. et al. (2003): IFN-lambdas mediate antiviral protection through a distinct class II cytokine receptor complex. In Nature immunology 4 (1), pp. 69-77. DOI: 10.1038/ni875.

Kraus, Thomas A.; Lau, Joe F.; Parisien, Jean-Patrick; Horvath, Curt M. (2003): A hybrid IRF9STAT2 protein recapitulates interferon-stimulated gene expression and antiviral response. In The Journal of biological chemistry 278 (15), pp. 13033-13038. DOI: 10.1074/jbc.M212972200.

Krug, Robert M. (1989): The Influenza Viruses. Boston, MA: Springer US (The Viruses). Available online at https://books.google.de/books?id=8JXhBwAAQBAJ.

Kumar, A.; Haque, J.; Lacoste, J.; Hiscott, J.; Williams, B. R. (1994): Double-stranded RNAdependent protein kinase activates transcription factor NF-kappa B by phosphorylating I kappa B. In Proceedings of the National Academy of Sciences of the United States of America 91 (14), pp. 6288-6292.

Kupke, Sascha Young; Riedel, Dietmar; Frensing, Timo; Zmora, Pawel; Reichl, Udo (2019): A Novel Type of Influenza A Virus-Derived Defective Interfering Particle with Nucleotide Substitutions in Its Genome. In Journal of virology 93 (4). DOI: 10.1128/JVI.01786-18.

Lau, Lincoln L. H.; Cowling, Benjamin J.; Fang, Vicky J.; Chan, Kwok-Hung; Lau, Eric H. Y.; Lipsitch, Marc et al. (2010): Viral shedding and clinical illness in naturally acquired influenza virus infections. In The Journal of infectious diseases 201 (10), pp. 1509-1516. DOI: $10.1086 / 652241$.

Lazear, Helen M.; Schoggins, John W.; Diamond, Michael S. (2019): Shared and Distinct Functions of Type I and Type III Interferons. In Immunity 50 (4), pp.907-923. DOI: 10.1016/j.immuni.2019.03.025.

Lengyel, P. (1982): Biochemistry of interferons and their actions. In Annual review of biochemistry 51, pp. 251-282. DOI: 10.1146/annurev.bi.51.070182.001343.

Lenschow, Deborah J.; Lai, Caroline; Frias-Staheli, Natalia; Giannakopoulos, Nadia V.; Lutz, Andrew; Wolff, Thorsten et al. (2007): IFN-stimulated gene 15 functions as a critical antiviral molecule against influenza, herpes, and Sindbis viruses. In Proceedings of the National Academy of Sciences of the United States of America 104 (4), pp. 1371-1376. DOI: 10.1073/pnas.0607038104.

Leppert, M. (1977): Further characterization of sendai virus DI-RNAs: A model for their generation. In Cell 12 (2), pp. 539-552. DOI: 10.1016/0092-8674(77)90130-1.

Levy, David E.; Darnell, J. E. (2002): Stats: transcriptional control and biological impact. In Nature reviews. Molecular cell biology 3 (9), pp. 651-662. DOI: 10.1038/nrm909.

Li, T.; Pattnaik, A. K. (1997): Replication signals in the genome of vesicular stomatitis virus and its defective interfering particles: identification of a sequence element that enhances DI RNA replication. In Virology 232 (2), pp. 248-259. DOI: 10.1006/viro.1997.8571.

Li, Zhengtu; Li, Li; Zhao, Shuai; Li, Jing; Zhou, Hongxia; Zhang, Yunhui et al. (2018): Reunderstanding anti-influenza strategy: attach equal importance to antiviral and anti- 
inflammatory therapies. In Journal of thoracic disease 10 (Suppl 19), S2248-S2259. DOI: 10.21037/jtd.2018.03.169.

Liao, Laura E.; Iwami, Shingo; Beauchemin, Catherine A. A. (2016): (In)validating experimentally derived knowledge about influenza $A$ defective interfering particles. In Journal of the Royal Society, Interface 13 (124). DOI: 10.1098/rsif.2016.0412.

Liu, Baoming; Li, Nan L.; Shen, Yang; Bao, Xiaoyong; Fabrizio, Thomas; Elbahesh, Husni et al. (2016): The C-Terminal Tail of TRIM56 Dictates Antiviral Restriction of Influenza A and B Viruses by Impeding Viral RNA Synthesis. In Journal of virology 90 (9), pp. 4369-4382. DOI: 10.1128/JVI.03172-15.

Liu, Su-Yang; Aliyari, Roghiyh; Chikere, Kelechi; Li, Guangming; Marsden, Matthew D.; Smith, Jennifer K. et al. (2012): Interferon-Inducible Cholesterol-25-Hydroxylase Broadly Inhibits Viral Entry by Production of 25-Hydroxycholesterol. In Immunity 38 (1), pp. 92-105. DOI: 10.1016/j.immuni.2012.11.005.

Lohr, V.; Rath, A.; Genzel, Y.; Jordan, I.; Sandig, V.; Reichl, U. (2009): New avian suspension cell lines provide production of influenza virus and MVA in serum-free media: studies on growth, metabolism and virus propagation. In Vaccine 27 (36), pp.4975-4982. DOI: 10.1016/j.vaccine.2009.05.083.

Lu, Pu-Xuan; Jing, Yuan; Xiao-Hua, Le; Zhou, Bo-Ping; Yu-Xin, Shi; Zhi-Yong, Zhang et al. (2016): Human Avian Influenza A H5N1, H7N9, H10N8 and H5N6 Virus Infection. In Pu-Xuan Lu, Bo-Ping Zhou (Eds.): Diagnostic imaging of emerging infectious diseases. Dordrecht: Springer, pp. 29-56.

Magnus, Preben von (1954): Incomplete Forms of Influenza Virus. In Max A. Lauffer, Kenneth M. Smith (Eds.): Advances in virus research, vol. 2. New York: Academic Press (Advances in Virus Research, v. 2), pp. 59-79.

Mallia, Patrick; Johnston, Sebastian L. (2007): Influenza infection and COPD. In International journal of chronic obstructive pulmonary disease 2 (1), pp.55-64. DOI: 10.2147/copd.2007.2.1.55.

Manicassamy, Balaji; Manicassamy, Santhakumar; Belicha-Villanueva, Alan; Pisanelli, Giuseppe; Pulendran, Bali; García-Sastre, Adolfo (2010): Analysis of in vivo dynamics of influenza virus infection in mice using a GFP reporter virus. In PNAS 107 (25), pp. 1153111536. DOI: 10.1073/pnas.0914994107.

Marcos-Villar, Laura; Díaz-Colunga, Juan; Sandoval, Juan; Zamarreño, Noelia; LanderasBueno, Sara; Esteller, Manel et al. (2018): Epigenetic control of influenza virus: role of H3K79 methylation in interferon-induced antiviral response. In Scientific reports 8 (1), p. 1230. DOI: 10.1038/s41598-018-19370-6.

Marriott, A. C.; Dimmock, N. J. (2010): Defective interfering viruses and their potential as antiviral agents. In Reviews in medical virology 20 (1), pp. 51-62. DOI: 10.1002/rmv.641.

Martínez-Sobrido, Luis; García-Sastre, Adolfo (2010): Generation of recombinant influenza virus from plasmid DNA. In Journal of visualized experiments : JoVE (42). DOI: 10.3791/2057. 
Matsuzaki, Yoko; Sugawara, Kanetsu; Furuse, Yuki; Shimotai, Yoshitaka; Hongo, Seiji; Oshitani, Hitoshi et al. (2016): Genetic Lineage and Reassortment of Influenza C Viruses Circulating between 1947 and 2014. In Journal of virology 90 (18), pp. 8251-8265. DOI: 10.1128/JVI.00969-16.

McNally, Beth; Ye, Fang; Willette, Meredith; Flaño, Emilio (2013): Local Blockade of Epithelial PDL-1 in the Airways Enhances T Cell Function and Viral Clearance during Influenza Virus Infection. In Journal of virology 87 (23), pp. 12916-12924. DOI: 10.1128/JVI.02423-13.

Meng, Bo; Bentley, Kirsten; Marriott, Anthony C.; Scott, Paul D.; Dimmock, Nigel J.; Easton, Andrew J. (2017): Unexpected complexity in the interference activity of a cloned influenza defective interfering RNA. In Virology journal 14 (1), p. 138. DOI: 10.1186/s12985-017-08056.

Meyerson, Nicholas R.; Zhou, Ligang; Guo, Yusong R.; Zhao, Chen; Tao, Yizhi J.; Krug, Robert M.; Sawyer, Sara L. (2017): Nuclear TRIM25 Specifically Targets Influenza Virus Ribonucleoproteins to Block the Onset of RNA Chain Elongation. In Cell host \& microbe 22 (5), 627-638.e7. DOI: 10.1016/j.chom.2017.10.003.

Milián, Ernest; Kamen, Amine A. (2015): Current and Emerging Cell Culture Manufacturing Technologies for Influenza Vaccines. In BioMed Research International 2015, pp. 1-11. DOI: $10.1155 / 2015 / 504831$.

Monto, A. S.; Gravenstein, S.; Elliott, M.; Colopy, M.; Schweinle, J. (2000): Clinical signs and symptoms predicting influenza infection. In Archives of internal medicine 160 (21), pp. 32433247. DOI: 10.1001/archinte.160.21.3243.

Mordstein, Markus; Neugebauer, Eva; Ditt, Vanessa; Jessen, Birthe; Rieger, Toni; Falcone, Valeria et al. (2010): Lambda interferon renders epithelial cells of the respiratory and gastrointestinal tracts resistant to viral infections. In Journal of virology 84 (11), pp. 56705677. DOI: 10.1128/JVI.00272-10.

Morlighem, Jean-Étienne; Aoki, Shintaro; Kishima, Mami; Hanami, Mitsue; Ogawa, Chihiro; Jalloh, Amadu et al. (2011): Mutation Analysis of 2009 Pandemic Influenza A(H1N1) Viruses Collected in Japan during the Peak Phase of the Pandemic. In PloS one 6 (4). DOI: 10.1371/journal.pone.0018956.

Moscona, Anne (1991): Defective interfering particles of human parainfluenza virus type 3 are associated with persistent infection in cell culture. In Virology 183 (2), pp. 821-824. DOI: 10.1016/0042-6822(91)91018-C.

Mura, Marie; Combredet, Chantal; Najburg, Valérie; Sanchez David, Raul Y.; Tangy, Frédéric; Komarova, Anastassia V. (2017): Nonencapsidated 5' Copy-Back Defective Interfering Genomes Produced by Recombinant Measles Viruses Are Recognized by RIG-I and LGP2 but Not MDA5. In Journal of virology 91 (20). DOI: 10.1128/JVI.00643-17.

Najjar, Imen; Fagard, Remi (2010): STAT1 and pathogens, not a friendly relationship. In Biochimie 92 (5), pp. 425-444. DOI: 10.1016/j.biochi.2010.02.009.

Nayak, D. P. (1980): Defective interfering influenza viruses. In Annual review of microbiology 34, pp. 619-644. DOI: 10.1146/annurev.mi.34.100180.003155. 
Nayak, D. P.; Chambers, T. M.; Akkina, R. K. (1985): Defective-interfering (DI) RNAs of influenza viruses: origin, structure, expression, and interference. In Current topics in microbiology and immunology 114, pp. 103-151. DOI: 10.1007/978-3-642-70227-3_3.

Nayak, D. P.; Sivasubramanian, N.; Davis, A. R.; Cortini, R.; Sung, J. (1982): Complete sequence analyses show that two defective interfering influenza viral RNAs contain a single internal deletion of a polymerase gene. In Proceedings of the National Academy of Sciences of the United States of America 79 (7), pp. 2216-2220. DOI: 10.1073/pnas.79.7.2216.

Nayak, D. P.; Tobita, K.; Janda, J. M.; Davis, A. R.; De, B. K. (1978): Homologous interference mediated by defective interfering influenza virus derived from a temperature-sensitive mutant of influenza virus. In Journal of virology 28 (1), pp. 375-386.

Neumann, G.; Watanabe, T.; Ito, H.; Watanabe, S.; Goto, H.; Gao, P. et al. (1999): Generation of influenza A viruses entirely from cloned cDNAs. In Proceedings of the National Academy of Sciences of the United States of America 96 (16), pp.9345-9350. DOI: 10.1073/pnas.96.16.9345.

Neumann, Gabriele; Noda, Takeshi; Kawaoka, Yoshihiro (2009): Emergence and pandemic potential of swine-origin H1N1 influenza virus. In Nature 459 (7249), pp. 931-939. DOI: $10.1038 /$ nature08157.

Ngunjiri, John M.; Lee, Chang-Won; Ali, Ahmed; Marcus, Philip I. (2012): Influenza virus interferon-inducing particle efficiency is reversed in avian and mammalian cells, and enhanced in cells co-infected with defective-interfering particles. In Journal of interferon \& cytokine research : the official journal of the International Society for Interferon and Cytokine Research 32 (6), pp. 280-285. DOI: 10.1089/jir.2011.0102.

Nogales, Aitor; Baker, Steven F.; Martínez-Sobrido, Luis (2015): Replication-competent influenza A viruses expressing a red fluorescent protein. In Virology 476, pp. 206-216. DOI: 10.1016/j.virol.2014.12.006.

Nogales, Aitor; Martínez-Sobrido, Luis (2016): Reverse Genetics Approaches for the Development of Influenza Vaccines. In International journal of molecular sciences 18 (1). DOI: $10.3390 /$ ijms18010020.

Odendall, Charlotte; Voak, Andrew A.; Kagan, Jonathan C. (2017): Type III IFNs Are Commonly Induced by Bacteria-Sensing TLRs and Reinforce Epithelial Barriers during Infection. In Journal of immunology (Baltimore, Md. : 1950) 199 (9), pp. 3270-3279. DOI: 10.4049/jimmunol.1700250.

Ohmit, Suzanne E.; Monto, Arnold S. (2006): Symptomatic predictors of influenza virus positivity in children during the influenza season. In Clinical infectious diseases : an official publication of the Infectious Diseases Society of America 43 (5), pp. 564-568. DOI: $10.1086 / 506352$.

Osterlund, Pamela I.; Pietilä, Taija E.; Veckman, Ville; Kotenko, Sergei V.; Julkunen, Ilkka (2007): IFN regulatory factor family members differentially regulate the expression of type III IFN (IFN-lambda) genes. In Journal of immunology (Baltimore, Md. : 1950) 179 (6), pp. 34343442. DOI: 10.4049/jimmunol.179.6.3434. 
Park, Christopher; Li, Suzanne; Cha, Edward; Schindler, Christian (2000): Immune Response in Stat2 Knockout Mice. In Immunity 13 (6), pp.795-804. DOI: 10.1016/S10747613(00)00077-7.

Pathak, Kunj B.; Nagy, Peter D. (2009): Defective Interfering RNAs: Foes of Viruses and Friends of Virologists. In Viruses 1 (3), pp. 895-919. DOI: 10.3390/v1030895.

Patil, Girish; Zhao, Mengmeng; Song, Kun; Hao, Wenzhuo; Bouchereau, Daniel; Wang, Lingyan; Li, Shitao (2018): TRIM41-Mediated Ubiquitination of Nucleoprotein Limits Influenza A Virus Infection. In Journal of virology 92 (16). DOI: 10.1128/JVI.00905-18.

Paules, Catharine; Subbarao, Kanta (2017): Influenza. In The Lancet 390 (10095), pp. 697708. DOI: 10.1016/S0140-6736(17)30129-0.

Paun, A.; Pitha, P. M. (2007): The IRF family, revisited. In Biochimie 89 (6-7), pp. 744-753. DOI: 10.1016/j.biochi.2007.01.014.

Perrault, J. (1981): Origin and replication of defective interfering particles. In Current topics in microbiology and immunology 93, pp. 151-207. DOI: 10.1007/978-3-642-68123-3_7.

Pestka, Sidney; Kotenko, Serguei V.; Muthukumaran, Geetha; Izotova, Lara S.; Cook, Jeffry R.; Garotta, Gianni (1997): The interferon gamma (IFN- $\gamma$ ) receptor: a paradigm for the multichain cytokine receptor. In Cytokine \& Growth Factor Reviews 8 (3), pp. 189-206. DOI: 10.1016/S1359-6101(97)00009-9.

Pestka, Sidney; Krause, Christopher D.; Walter, Mark R. (2004): Interferons, interferon-like cytokines, and their receptors. In Immunological reviews 202, pp. 8-32. DOI: 10.1111/j.01052896.2004.00204.x.

Pichlmair, Andreas; Schulz, Oliver; Tan, Choon Ping; Näslund, Tanja I.; Liljeström, Peter; Weber, Friedemann; Reis e Sousa, Caetano (2006): RIG-I-mediated antiviral responses to single-stranded RNA bearing 5'-phosphates. In Science (New York, N.Y.) 314 (5801), pp. 9971001. DOI: $10.1126 /$ science.1132998.

Public Health England (2019): PHE guidance on use of antiviral agents for the treatment and prophylaxis of influenza. Available online at https://assets.publishing.service.gov.uk/government/uploads/system/uploads/attachment_ data/file/833572/PHE_guidance_antivirals_influenza_201920.pdf.

Qu, Hongren; Li, Jing; Yang, Limin; Sun, Lei; Liu, Wenjun; He, Hongxuan (2016): Influenza A Virus-induced expression of ISG20 inhibits viral replication by interacting with nucleoprotein. In Virus genes 52 (6), pp. 759-767. DOI: 10.1007/s11262-016-1366-2.

Rabinowitz, S. G.; Huprikar, J. (1979): The influence of defective-interfering particles of the PR-8 strain of influenza A virus on the pathogenesis of pulmonary infection in mice. In The Journal of infectious diseases 140 (3), pp. 305-315. DOI: 10.1093/infdis/140.3.305.

Ramsay, Mary (2019): Greenbook of Immunisation: chapter 19 - Influenza. Available online at https://assets.publishing.service.gov.uk/government/uploads/system/uploads/attach ment_data/file/796886/GreenBook_Chapter_19_Influenza_April_2019.pdf. 
Rappuoli, Rino (2006): Cell-culture-based vaccine production: technological options. In BRIDGE-WASHINGTON-NATIONAL ACADEMY OF ENGINEERING- 36 (3), p. 25.

Reid, Elizabeth; Juleff, Nicholas; Windsor, Miriam; Gubbins, Simon; Roberts, Lisa; Morgan, Sophie et al. (2016): Type I and III IFNs Produced by Plasmacytoid Dendritic Cells in Response to a Member of the Flaviviridae Suppress Cellular Immune Responses. In Journal of immunology (Baltimore, Md. : 1950) 196 (10), pp.4214-4226. DOI: 10.4049/jimmunol.1600049.

Sadler, A. J.; Williams, B. R. G. (2007): Structure and function of the protein kinase R. In Current topics in microbiology and immunology 316, pp. 253-292. DOI: 10.1007/978-3-54071329-6_13.

Saira, Kazima; Lin, Xudong; DePasse, Jay V.; Halpin, Rebecca; Twaddle, Alan; Stockwell, Timothy et al. (2013): Sequence analysis of in vivo defective interfering-like RNA of influenza A H1N1 pandemic virus. In Journal of virology 87 (14), pp. 8064-8074. DOI: 10.1128/JVI.00240-13.

Schindler, C.; Shuai, K.; Prezioso, V. R.; Darnell, J. E. (1992): Interferon-dependent tyrosine phosphorylation of a latent cytoplasmic transcription factor. In Science (New York, N.Y.) 257 (5071), pp. 809-813. DOI: 10.1126/science.1496401.

Schmid, Sonja; Mordstein, Markus; Kochs, Georg; García-Sastre, Adolfo; tenOever, Benjamin R. (2010): Transcription factor redundancy ensures induction of the antiviral state. In The Journal of biological chemistry 285 (53), pp. 42013-42022. DOI: 10.1074/jbc.M110.165936.

Schneider, William M.; Chevillotte, Meike Dittmann; Rice, Charles M. (2014): Interferonstimulated genes: a complex web of host defenses. In Annual review of immunology 32, pp. 513-545. DOI: 10.1146/annurev-immunol-032713-120231.

Schoggins, John W.; Wilson, Sam J.; Panis, Maryline; Murphy, Mary Y.; Jones, Christopher T.; Bieniasz, Paul; Rice, Charles M. (2011): A diverse range of gene products are effectors of the type I interferon antiviral response. In Nature 472 (7344), pp.481-485. DOI: 10.1038/nature09907.

Schubert, M.; Lazzarini, R. A. (1981): Structure and origin of a snapback defective interfering particle RNA of vesicular stomatitis virus. In Journal of virology 37 (2), pp. 661-672.

Schulz, Oliver; Pichlmair, Andreas; Rehwinkel, Jan; Rogers, Neil C.; Scheuner, Donalyn; Kato, Hiroki et al. (2010): Protein kinase $R$ contributes to IFN- $\alpha / \beta$ production during viral infection by regulating IFN mRNA integrity. In Cell host \& microbe 7 (5), pp. 354-361. DOI: 10.1016/j.chom.2010.04.007.

Scott, Paul D.; Meng, Bo; Marriott, Anthony C.; Easton, Andrew J.; Dimmock, Nigel J. (2011a): Defective interfering influenza $A$ virus protects in vivo against disease caused by a heterologous influenza B virus. In The Journal of general virology 92 (Pt 9), pp. 2122-2132. DOI: 10.1099/vir.0.034132-0.

Scott, Paul D.; Meng, Bo; Marriott, Anthony C.; Easton, Andrew J.; Dimmock, Nigel J. (2011b): Defective interfering influenza virus confers only short-lived protection against influenza 
virus disease: evidence for a role for adaptive immunity in DI virus-mediated protection in vivo. In Vaccine 29 (38), pp. 6584-6591. DOI: 10.1016/j.vaccine.2011.06.114.

Scott, Paul D.; Meng, Bo; Marriott, Anthony C.; Easton, Andrew J.; Dimmock, Nigel J. (2011c): Defective interfering virus protects elderly mice from influenza. In Virology journal 8, p. 212. DOI: 10.1186/1743-422X-8-212.

Seitz, Claudius; Isken, Britta; Heynisch, Björn; Rettkowski, Maria; Frensing, Timo; Reichl, Udo (2012): Trypsin promotes efficient influenza vaccine production in MDCK cells by interfering with the antiviral host response. In Applied microbiology and biotechnology 93 (2), pp. 601611. DOI: 10.1007/s00253-011-3569-8.

Seth, Rashu B.; Sun, Lijun; Ea, Chee-Kwee; Chen, Zhijian J. (2005): Identification and characterization of MAVS, a mitochondrial antiviral signaling protein that activates NFkappaB and IRF 3. In Cell 122 (5), pp. 669-682. DOI: 10.1016/j.cell.2005.08.012.

Shaw, M. L.; Palese, P. (2013): Fields virology, p 1151-1185. In Fields virology, 6th ed. Lippincott Williams \& Wilkins, Philadelphia, PA.

Sheppard, Paul; Kindsvogel, Wayne; Xu, Wenfeng; Henderson, Katherine; Schlutsmeyer, Stacy; Whitmore, Theodore E. et al. (2003): IL-28, IL-29 and their class II cytokine receptor IL28R. In Nature immunology 4 (1), pp. 63-68. DOI: 10.1038/ni873.

Siegal, F. P.; Kadowaki, N.; Shodell, M.; Fitzgerald-Bocarsly, P. A.; Shah, K.; Ho, S. et al. (1999): The nature of the principal type 1 interferon-producing cells in human blood. In Science (New York, N.Y.) 284 (5421), pp. 1835-1837. DOI: 10.1126/science.284.5421.1835.

Silverman, Robert H. (2007): Viral Encounters with 2',5'-Oligoadenylate Synthetase and RNase L during the Interferon Antiviral Response ${ }^{\nabla}$. In Journal of virology 81 (23), pp. 1272012729. DOI: 10.1128/JVI.01471-07.

Sommereyns, Caroline; Paul, Sophie; Staeheli, Peter; Michiels, Thomas (2008): IFN-lambda (IFN-lambda) is expressed in a tissue-dependent fashion and primarily acts on epithelial cells in vivo. In PLoS pathogens 4 (3), e1000017. DOI: 10.1371/journal.ppat.1000017.

Stark, G. R.; Kerr, I. M.; Williams, B. R.; Silverman, R. H.; Schreiber, R. D. (1998): How cells respond to interferons. In Annual review of biochemistry 67, pp. 227-264. DOI: 10.1146/annurev.biochem.67.1.227.

Stark, George R.; Darnell, James E. (2012): The JAK-STAT pathway at twenty. In Immunity 36 (4), pp. 503-514. DOI: 10.1016/j.immuni.2012.03.013.

Stöhr, Klaus; Bucher, Doris; Colgate, Tony; Wood, John (2012): Influenza virus surveillance, vaccine strain selection, and manufacture. In Methods in molecular biology (Clifton, N.J.) 865, pp. 147-162. DOI: 10.1007/978-1-61779-621-0_9.

Te Velthuis, Aartjan J. W.; Fodor, Ervin (2016): Influenza virus RNA polymerase: insights into the mechanisms of viral RNA synthesis. In Nature reviews. Microbiology 14 (8), pp. 479-493. DOI: $10.1038 /$ nrmicro.2016.87.

Te Velthuis, Aartjan J. W.; Long, Joshua C.; Bauer, David L. V.; Fan, Rebecca L. Y.; Yen, HuiLing; Sharps, Jane et al. (2018): Mini viral RNAs act as innate immune agonists during 
influenza virus infection. In Nat Microbiol 3 (11), pp. 1234-1242. DOI: 10.1038/s41564-0180240-5.

Tong, Suxiang; Li, Yan; Rivailler, Pierre; Conrardy, Christina; Castillo, Danilo A. Alvarez; Chen, Li-Mei et al. (2012): A distinct lineage of influenza A virus from bats. In Proceedings of the National Academy of Sciences of the United States of America 109 (11), pp. 4269-4274. DOI: 10.1073/pnas.1116200109.

Tong, Suxiang; Zhu, Xueyong; Li, Yan; Shi, Mang; Zhang, Jing; Bourgeois, Melissa et al. (2013): New world bats harbor diverse influenza A viruses. In PLoS pathogens 9 (10), e1003657. DOI: 10.1371/journal.ppat.1003657.

Troeger, Christopher E.; Blacker, Brigette F.; Khalil, Ibrahim A.; Zimsen, Stephanie R. M.; Albertson, Samuel B.; Abate, Degu et al. (2019): Mortality, morbidity, and hospitalisations due to influenza lower respiratory tract infections, 2017: an analysis for the Global Burden of Disease Study 2017. In The Lancet Respiratory Medicine 7 (1), pp.69-89. DOI: 10.1016/S2213-2600(18)30496-X.

van den Hoogen, Bernadette G.; van Boheemen, Sander; Rijck, Jonneke de; van Nieuwkoop, Stefan; Smith, Derek J.; Laksono, Brigitta et al. (2014): Excessive production and extreme editing of human metapneumovirus defective interfering RNA is associated with type I IFN induction. In The Journal of general virology 95 (Pt 8), pp.1625-1633. DOI: 10.1099/vir.0.066100-0.

Vasilijevic, Jasmina; Zamarreño, Noelia; Oliveros, Juan Carlos; Rodriguez-Frandsen, Ariel; Gómez, Guillermo; Rodriguez, Guadalupe et al. (2017): Reduced accumulation of defective viral genomes contributes to severe outcome in influenza virus infected patients. In PLOS pathogens 13 (10), e1006650. DOI: 10.1371/journal.ppat.1006650.

Velazquez, Laura; Fellous, Marc; Stark, George R.; Pellegrini, Sandra (1992): A protein tyrosine kinase in the interferon $\alpha \beta$ signaling pathway. In Cell 70 (2), pp. 313-322. DOI: 10.1016/0092-8674(92)90105-L.

Vignuzzi, Marco; López, Carolina B. (2019): Defective viral genomes are key drivers of the virus-host interaction. In Nat Microbiol 4 (7), pp. 1075-1087. DOI: 10.1038/s41564-0190465-y.

Villalón-Letelier, Fernando; Brooks, Andrew G.; Saunders, Philippa M.; Londrigan, Sarah L.; Reading, Patrick C. (2017): Host Cell Restriction Factors that Limit Influenza A Infection. In Viruses 9 (12). DOI: 10.3390/v9120376.

Wang, Chang; Forst, Christian V.; Chou, Tsui-Wen; Geber, Adam; Wang, Minghui; Hamou, Wissam et al. (2020): Cell-to-Cell Variation in Defective Virus Expression and Effects on Host Responses during Influenza Virus Infection. In mBio 11 (1). DOI: 10.1128/mBio.02880-19.

Wang, Wenshi; Xu, Lei; Su, Junhong; Peppelenbosch, Maikel P.; Pan, Qiuwei (2017): Transcriptional Regulation of Antiviral Interferon-Stimulated Genes. In Trends in microbiology 25 (7), pp. 573-584. DOI: 10.1016/j.tim.2017.01.001. 
Wang, Xiuyan; Hinson, Ella R.; Cresswell, Peter (2007): The interferon-inducible protein viperin inhibits influenza virus release by perturbing lipid rafts. In Cell host \& microbe 2 (2), pp. 96-105. DOI: 10.1016/j.chom.2007.06.009.

Wasik, Milena A.; Eichwald, Luca; Genzel, Yvonne; Reichl, Udo (2018): Cell culture-based production of defective interfering particles for influenza antiviral therapy. In Applied microbiology and biotechnology 102 (3), pp. 1167-1177. DOI: 10.1007/s00253-017-8660-3.

Weber, Michaela; Gawanbacht, Ali; Habjan, Matthias; Rang, Andreas; Borner, Christoph; Schmidt, Anna Mareike et al. (2013): Incoming RNA virus nucleocapsids containing a 5'triphosphorylated genome activate RIG-I and antiviral signaling. In Cell host \& microbe 13 (3), pp. 336-346. DOI: 10.1016/j.chom.2013.01.012.

Weber-Gerlach, Michaela; Weber, Friedemann (2016): Standing on three legs: antiviral activities of RIG-I against influenza viruses. In Current opinion in immunology 42, pp. 71-75. DOI: 10.1016/j.coi.2016.05.016.

Webster, R. G.; Bean, W. J.; Gorman, O. T.; Chambers, T. M.; Kawaoka, Y. (1992): Evolution and ecology of influenza A viruses. In Microbiological reviews 56 (1), pp. 152-179.

White, K. A.; Morris, T. J. (1994): Nonhomologous RNA recombination in tombusviruses: generation and evolution of defective interfering RNAs by stepwise deletions. In Journal of virology 68 (1), pp. 14-24.

WHO (2020a): Global Influenza Surveillance and Response System (GISRS) (GISRS and laboratory). Available online at https://www.who.int/influenza/gisrs_laboratory/en/, checked on 4/5/2020.

WHO (2020b): Influenza (Seasonal). Available online at https://www.who.int/newsroom/fact-sheets/detail/influenza-(seasonal), checked on 4/5/2020.

WHO (2020c): Influenza vaccine viruses and reagents (Vaccine viruses). Available online at https://www.who.int/influenza/vaccines/virus/en/, checked on 4/5/2020.

Wu, Jiaxi; Chen, Zhijian J. (2014): Innate immune sensing and signaling of cytosolic nucleic acids. In Annual review of immunology 32, pp. 461-488. DOI: 10.1146/annurev-immunol032713-120156.

Xagorari, Angeliki; Chlichlia, Katerina (2008): Toll-like receptors and viruses: induction of innate antiviral immune responses. In The open microbiology journal 2, pp.49-59. DOI: $10.2174 / 1874285800802010049$.

Xue, Qinghua; Zhou, Zhuo; Lei, Xiaobo; Liu, Xinlei; He, Bin; Wang, Jianwei; Hung, Tao (2012): TRIM38 negatively regulates TLR3-mediated IFN- $\beta$ signaling by targeting TRIF for degradation. In PloS one 7 (10), e46825. DOI: 10.1371/journal.pone.0046825.

Yamagata, Yutaro; Muramoto, Yukiko; Miyamoto, Sho; Shindo, Keiko; Nakano, Masahiro; Noda, Takeshi (2019): Generation of a purely clonal defective interfering influenza virus. In Microbiology and immunology 63 (5), pp. 164-171. DOI: 10.1111/1348-0421.12681. 
Yang, Yicheng; Lyu, Taibiao; Zhou, Runing; He, Xiaoen; Ye, Kaiyan; Xie, Qian et al. (2019): The Antiviral and Antitumor Effects of Defective Interfering Particles/Genomes and Their Mechanisms. In Frontiers in microbiology 10, p. 1852. DOI: 10.3389/fmicb.2019.01852.

Yoneyama, Mitsutoshi; Onomoto, Koji; Jogi, Michihiko; Akaboshi, Teppei; Fujita, Takashi (2015): Viral RNA detection by RIG-I-like receptors. In Current opinion in immunology 32, pp. 48-53. DOI: 10.1016/j.coi.2014.12.012.

Zhang, Junsong; Huang, Feng; Tan, Likai; Bai, Chuan; Chen, Bing; Liu, Jun et al. (2016): Host Protein Moloney Leukemia Virus 10 (MOV10) Acts as a Restriction Factor of Influenza A Virus by Inhibiting the Nuclear Import of the Viral Nucleoprotein. In Journal of virology 90 (8), pp. 3966-3980. DOI: 10.1128/JVI.03137-15.

Zimmermann, Petra; Mänz, Benjamin; Haller, Otto; Schwemmle, Martin; Kochs, Georg (2011): The viral nucleoprotein determines Mx sensitivity of influenza A viruses. In Journal of virology 85 (16), pp. 8133-8140. DOI: 10.1128/JVI.00712-11. 


\section{Appendix}

\section{List of abbreviations}

\begin{tabular}{|c|c|}
\hline ATP & adenosine triphosphate \\
\hline cRNA & Complementary RNA \\
\hline DI RNAs & Defective interfering RNAs \\
\hline DIPs & Defective interfering particles \\
\hline DNA & Deoxy-ribonucleic acid \\
\hline GAF & Interferon-gamma activation factor \\
\hline GAS & Gamma-activated sequence \\
\hline GTP & Guanosine triphosphate \\
\hline HA & Hemagglutinin \\
\hline HIV & Human immunodeficiency virus \\
\hline IAV & Influenza A virus \\
\hline IBV & Influenza B virus \\
\hline IFITM & Interferon-induced transmembrane protein \\
\hline IFN & Interferon \\
\hline IRF & Interferon regulatory factor \\
\hline ISG & Interferon-stimulated genes \\
\hline ISGF3 & Interferon-stimulated gene factor 3 \\
\hline ISRE & Interferon-stimulated response element \\
\hline M1 & Matrix protein 1 \\
\hline M2 & Matrix protein 2 \\
\hline MAVS & Mitochondrial antiviral-signalling protein \\
\hline MDA5 & Melanoma differentiated-associated protein 5 gene \\
\hline MDCK & Madin-Darby Canine Kidney cells \\
\hline MOI & Multiplicity of infection \\
\hline mRNA & Messenger RNA \\
\hline $\mathrm{Mx}$ & Mxyovirus resistance gene \\
\hline NA & Neuraminidase \\
\hline NCR & Non-coding region \\
\hline NEP & Nuclear export protein \\
\hline
\end{tabular}


Nucleoprotein

NS1 Non-structural protein 1

NS2 Non-structural protein 2

PA Polymerase acidic

PAMPs Pathogen associated molecular patterns

PB1 Polymerase basic 1

PB2 Polymerase basic 2

PB2 opt Codon-optimized PB2

Pol-I RNA polymerase I

Pol-II Polymerase II cytomegalovirus promoter

PR8 A/Puerto Rico/8/34

PRRs Pattern recognition receptors

PVM Pneumonia virus of mice

RdRp RNA-dependant RNA polymerase

RIG-I Retinoic acid-inducible gene 1

RLRs RIG-I like receptors

RNA Ribonucleic acid

SARS-CoV Severe acute respiratory syndrome corona virus

SFV Semliki forest virus

STAT Signal transducers and activators of transcription

UV Ultra-violet

VRNA Viral RNA

VRNP Viral ribonucleoprotein

VSV Vesicular stomatitis virus

WHO World Health Organization

WSN $\quad$ A/WSN/33

wt Wild-type 


\section{Acknowledgements}

I would like to express my sincere gratitude to my supervisor Prof. Dr. Stefan Pöhlmann for his continuous motivation and immense understanding during my doctoral thesis. His guidance helped me throughout my research and shaped my study into the present form.

I would like to thank Michael for his support, guidance in planning and designing experiments, fruitful discussions and most importantly feedback on my thesis.

I would like to thank Markus for his help in framing experiments, statistical analysis, and prism figures.

Besides my supervisors, Prof. Dr. Lutz Walter, Late Prof. Dr. Dr. Claus-Peter Czerny and Prof. Friedemann Weber for accepting my request to be a part of my thesis committee and for their opinions and suggestions to improve my research work.

I would like to thank Kathrin for her kind gesture and organizational work. You were always there to lend a helping hand.

My sincere thanks to Sabine for her technical assistance. Her experience and skills helped me to keep this project live even under immense stress. Also, I would like to thank Stefanie for her help and liveliness in the lab.

I thank all the members of the infection biology unit individually. Thank you Inga for Western blot and DIP production, Anna for Maxi prep, Najat for being my DIP partner, Astrid for mycoplasma test, Heidi for helping fill my tip boxes, Betty for telling her stories of hobbies, Hannah for accompanying in conferences, Mariana for briefing me with GGNB regulations, Artur for career guidance, Heike for enthusiasm, Abdul for discussion on culture.

I would like to thank my friend circle in Göttingen and my best friend Rachita, who always cheered me up. My brother Anish and sister in law Sandra for always being there by my side. Dieter for his interactive German lessons. A special thanks to my friend Rahul for keeping me calm and relaxed. Also, for cooking delicious dishes and keeping me hydrated during stressful days of thesis writing. A big thanks to my parents who believed, trusted and always supported me seamlessly that I can achieve this honoured degree. 


\section{Conference Participations}

$28^{\text {th }}$ Annual Meeting of Society of Virology; 14-17 March 2018; Würzburg

Poster Presentation: Evidence that the antiviral activity of influenza A virus defective interfering particles (DIP) depends on length of the defective genome segment.

Negative Strand Viruses; 17-22 June 2018; Verona, Italy

Poster presentation: Identification of determinants that control antiviral activity of defective interfering particles (DIP) and generation of tools for DIP production.

$10^{\text {th }}$ anniversary of Göttingen Graduate School for Neurosciences, Biophysics and Molecular Biosciences (GGNB) "Science Day"; $16^{\text {th }}$ November 2018; Göttingen

Poster presentation: RNA length is the major determinant of antiviral activity of defective interfering RNAs

$29^{\text {th }}$ Annual Meeting of Society of Virology; 20-23 March 2019; Düsseldorf

Poster Presentation: Analysis of determinants controlling antiviral activity of defective interfering influenza A virus RNAs.

$18^{\text {th }}$ workshop on "Cell Biology of Viral Infections"; 23-25 October 2019; Schöntal

Oral presentation: Determinants controlling antiviral activity of defective interfering influenza A virus RNAs. 